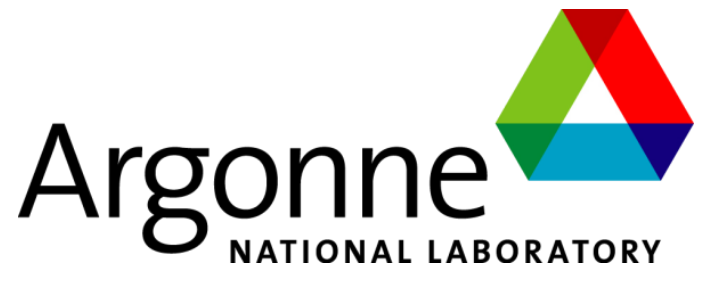

ANL/ESD-16/15

\title{
Investigation of Aerodynamic Interference between Twin Deck Bridges.
}

May 2016 


\title{
About Argonne National Laboratory
}

Argonne is a U.S. Department of Energy laboratory managed by UChicago Argonne, LLC under contract DE-AC02-06CH11357. The Laboratory's main facility is outside Chicago, at 9700 South Cass Avenue, Argonne, Illinois 60439. For information about Argonne and its pioneering science and technology programs, see www.anl.gov.

\section{DOCUMENT AVAILABILITY}

Online Access: U.S. Department of Energy (DOE) reports produced after 1991 and a growing number of pre-1991 documents are available free via DOE's SciTech Connect (http://www.osti.gov/scitech/)

\author{
Reports not in digital format may be purchased by the public from the \\ National Technical Information Service (NTIS): \\ U.S. Department of Commerce \\ National Technical Information Service \\ 5301 Shawnee Rd \\ Alexandra, VA 22312 \\ www.ntis.gov \\ Phone: (800) 553-NTIS (6847) or (703) 605-6000 \\ Fax: (703) 605-6900 \\ Email: orders@ntis.gov \\ Reports not in digital format are available to DOE and DOE contractors from the \\ Office of Scientific and Technical Information (OSTI): \\ U.S. Department of Energy \\ Office of Scientific and Technical Information \\ P.O. Box 62 \\ Oak Ridge, TN 37831-0062 \\ www.osti.gov \\ Phone: (865) 576-8401 \\ Fax: (865) 576-5728 \\ Email: reports@osti.gov
}

Disclaimer

This report was prepared as an account of work sponsored by an agency of the United States Government. Neither the United States Government nor any agency thereof, nor UChicago Argonne, LLC, nor any of their employees or officers, makes any warranty, express or implied, or assumes any legal liability or responsibility for the accuracy, completeness, or usefulness of any information, apparatus, product, or process disclosed, or represents that its use would not infringe privately owned rights. Reference herein to any specific commercial product, process, or service by trade name, trademark, manufacturer, or otherwise, does not necessarily constitute or imply its endorsement, recommendation, or favoring by the United States Government or any agency thereof. The views and opinions of document authors expressed herein do not necessarily state or reflect those of the United States Government or any agency thereof, Argonne National Laboratory, or UChicago Argonne, LLC. 


\section{Investigation of Aerodynamic Interference between Twin Deck Bridges.}

by

M.A. Sitek, C. Bojanowski, and S.A. Lottes

Transportation Research and Analysis Computing Center (TRACC)

Energy Systems Division, Argonne National Laboratory

May 2016 
Table of Contents

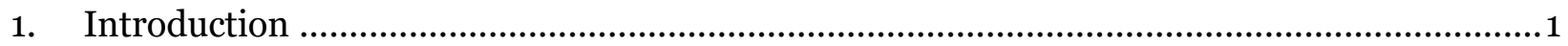

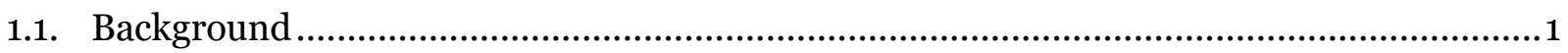

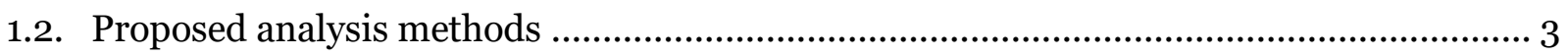

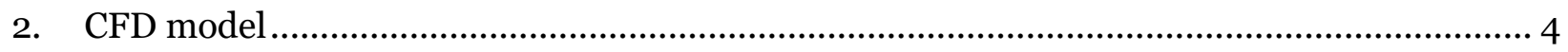

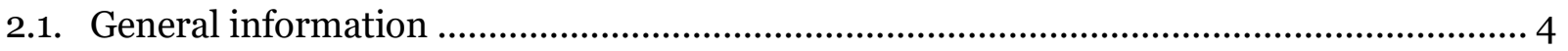

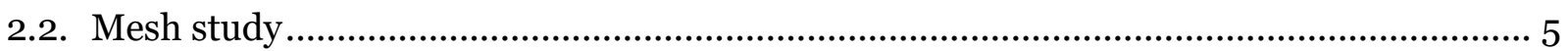

3. Analysis of the flow around a single deck bridge .............................................................10

4. Analysis of the flow around a twin deck bridge........................................................... 17

4.1. Pressure acting on a twin deck bridge ..................................................................... 17

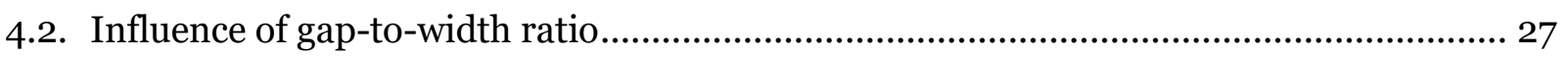

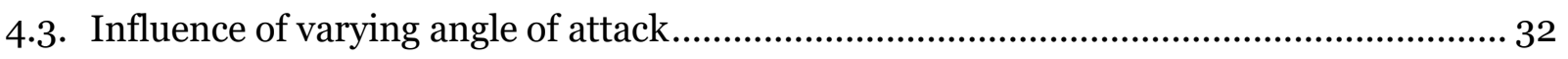

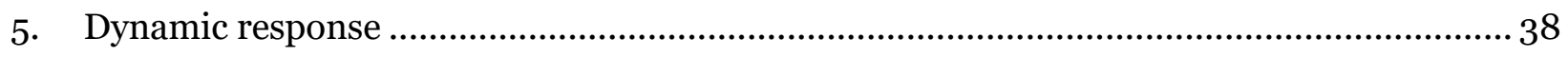

5.1. Configuration 1: both decks suspended on springs ................................................... 39

5.2. Configuration 2: the upstream deck is suspended on springs, the downstream deck is

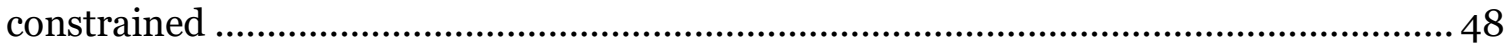

5.3. Configuration 3: the upstream deck is constrained, the downstream deck is suspended

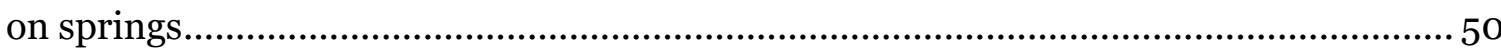

5.4. Comparison of results for configurations 1, 2, and 3............................................... 51

6. Validation of the CFD modeling approach ................................................................. 54

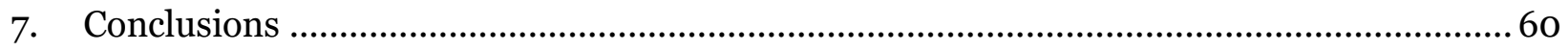

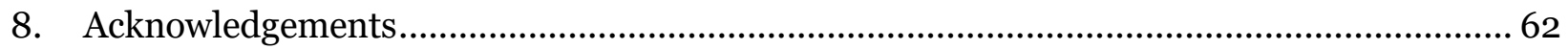

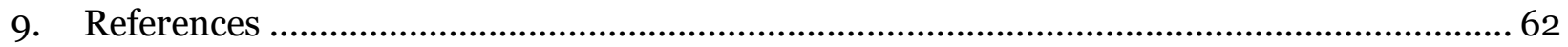

Investigation of Aerodynamic Interference between Twin Deck Bridges I I 


\section{List of Figures}

Figure 1-1: Thaddeus Kosciusko Bridge, NY, by Njhepler at English Wikipedia, CC BY 2.5, https://commons.wikimedia.org/w/index.php?curid=12452015

Figure 1-2: Fred Hartman Bridge, TX, by United States Coast Guard, PA2 James Dillard - U.S. Coast Guard Visual Information GalleryU.S. Coast Guard Visual Information Gallery Home, Public Domain, https://commons.wikimedia.org/w/index.php?curid=3499375 ..................... 2

Figure 2-1: The computational domain with twin deck dimensions in meters and locations of local CSYS origins

Figure 2-2: An example mesh around single and twin deck cross sections

Figure 2-3: Force component values vs. wall $\mathrm{Y}+(\mathrm{P}-$ polyhedral mesh, $\mathrm{H}-$ hexahedral mesh, ratio - stretch factor for the cell thickness in the prism layer)

Figure 2-4: Pitch moment component values vs. $\mathrm{Y}+(\mathrm{P}-$ polyhedral mesh, $\mathrm{H}-$ hexahedral mesh, ratio - stretch factor for the cell thickness in the prism layer)................................... 8

Figure 2-5: Mesh influence on force and moment components values acting on the upstream deck

Figure 2-6: Mesh influence on force and moment components values acting on the downstream deck.

Figure 3-1: Single deck. Pressure plot on the mid section of the domain (a) zoomed out view, (b) the area around the deck

Figure 3-2: Single deck. Horizontal velocity plot on the mid section of the domain (a) general view, (b) the area around the deck.

Figure 4-1: Pressure contour plot around the decks, (a) URANS, (b) LES at an instant in time, polyhedral mesh, $\mathrm{Y}+=1$.

Figure 4-2: Pressure on the symmetry line of the top surface of upstream deck, (a) location of measured points, (b) pressure values

Figure 4-3: Pressure on the symmetry line of the bottom surface of the upstream deck, (a) location of measured points, (b) pressure values 20

Figure 4-4: Pressure on the symmetry line of the top surface of downstream deck, (a) location of measured points, (b) pressure values

Figure 4-5: Pressure on the symmetry line of the bottom surface of downstream deck, (a) location of measured points, (b) pressure values 
Figure 4-6: Pressure on the symmetry line of the upstream surface of the upstream deck, (a) location of measured points, (b) pressure values

Figure 4-7: Pressure on the symmetry line of the downstream surface of upstream deck, (a) location of measured points, (b) pressure values

Figure 4-8: Pressure on the symmetry line of the upstream surface of the downstream deck, (a) location of measured points, (b) pressure values 25

Figure 4-9: Pressure on the symmetry line of the downstream surface of the downstream deck, (a) location of measured points, (b) pressure values. 26

Figure 4-10: Gap-to width ratio influence on the drag force in parallel flow. Deck 1 - upstream deck, deck 2 - downstream deck.

Figure 4-11: Gap-to width ratio influence on the lift force in parallel flow. Deck 1 - upstream deck, deck 2 - downstream deck.

Figure 4-12: Gap-to width ratio influence on the pitch moment in parallel flow. Deck 1 upstream deck, deck 2 - downstream deck.

Figure 4-13: Influence of angle of attack on drag force, L/B=0.13. Deck 1 - upstream deck, deck 2 - downstream deck.

Figure 4-14: Influence of angle of attack on lift force, L/B=0.13. Deck 1 - upstream deck, deck 2 - downstream deck.

Figure 4-15: Influence of angle of attack on pitch moment, $\mathrm{L} / \mathrm{B}=0.13$. Deck 1 - upstream deck, deck 2 - downstream deck.

Figure 5-1: Twin deck model on elastic springs.

Figure 5-2: Vertical translations of the decks in time, (a) during the entire simulation, (b) during last 5 seconds of the simulation time.

Figure 5-3: Fast Fourier Transform of the vertical vibrations of the decks

Figure 5-4: Rotations of the decks in time, (a) during the entire simulation, (b) during last 5 seconds of the simulation time.

Figure 5-5: Fast Fourier Transform of the rotational vibrations of the decks 42

Figure 5-6: Forces acting on the decks in configuration 1, (a) drag, (b) lift and (c) moment ...... 43

Figure 5-7: Forces and moment acting on the decks in configuration 2, (a) drag force, (b) lift force, (c) moment.

Figure 5-8: Forces and moment acting on the decks in configuration 3, (a) drag force, (b) lift force, (c) moment. 
Figure 5-9: Comparison of force values acting on deck 1 at different configurations .................. 52

Figure 5-10: Comparison of force values acting on deck 2 at different configurations............... 53

Figure 2-1: Stonecutters bridge cross-section. All dimensions are given in meters................... 54

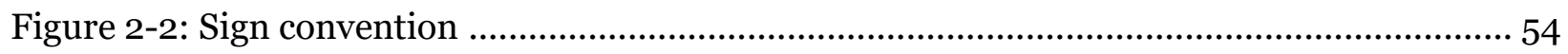

Figure 2-3: Sensitivity of force coefficients to Reynolds number.......................................... 55

Figure 2-4: The drag coefficient at different angles of attack............................................ 56

Figure 2-5: The lift coefficient at different angles of attack .............................................. 56

Figure 2-6: The pitch moment coefficient at different angles of attack ....................................57

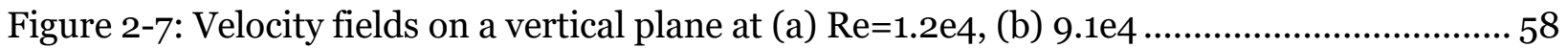

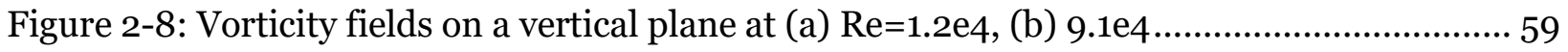

Investigation of Aerodynamic Interference between Twin Deck Bridges $\quad$ Page IV 
List of Tables

Table 2-1. Types of meshes used in the simulations....................................................... 6

Table 3-1: Pressure field contour plots for a single deck bridge at varying angle of attack ..........13

Table 3-2: Velocity field contour plots for a single deck bridge at varying angle of attack ...........15

Table 3-3: Extreme values of pressure and velocity on a single deck......................................17

Table 4-1: Extreme values of pressure and velocity for a twin deck with varying gap ............... 27

Table 4-2: Pressure and velocity fields around twin deck with varying gap ............................ 27

Table 4-3: Velocity field contour plots for a twin deck bridge at varying gap ............................29

Table 4-4: Pressure field contour plots for a twin deck bridge with $0.1 \mathrm{~m}$ gap $(\mathrm{L} / \mathrm{B}=0.26)$ at

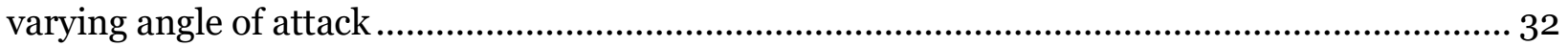

Table 4-5: Velocity field contour plots for a twin deck bridge with $0.1 \mathrm{~m}$ gap $(\mathrm{L} / \mathrm{B}=0.26)$ at

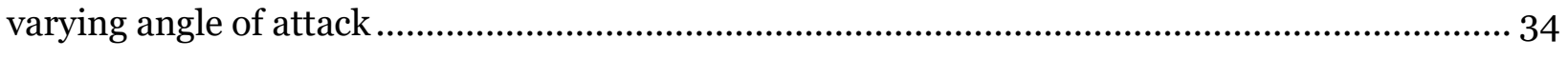

Table 4-6: Extreme values of pressure and velocity for a twin deck ....................................... 36

Table 5-1. Comparison of drag force, lift force and pitch moment for a stationary twin deck and a twin deck suspended on elastic springs ................................................................................. 44

Table 5-2: Velocity vector field on the middle plane in consecutive time steps......................... 44

Table 5-3: Velocity streamlines in consecutive time steps ................................................... 46

Table 2-1: Force coefficients and Strouhal number obtained from CFD simulations................. 59

Investigation of Aerodynamic Interference between Twin Deck Bridges $\quad$ Page V 


\section{Introduction}

\subsection{Background}

Construction of a twin bridge can be a cost effective and minimally disruptive way to increase capacity when an existing bridge is not near the end of its service life. With ever growing vehicular traffic, the demand approaches the capacity of many existing roads and bridges. Remodeling a structure with an insufficient number of lanes can be a good solution in case of smaller and less busy bridges. Closing down or reducing traffic on crossings of greater importance for the construction period, however, can result in major delays and revenue loss for commerce and transportation as well as increasing the traffic load on alternate routes. Multiple-deck bridges are considered to be the answer to this issue. A parallel deck can be built next to the existing one without reducing the flow. Moreover, it can be decided on the design stage that a new bridge has a twin or multi-deck structure.

Several such structures have already been built throughout the United States, among them: the new NY Bridge Project - the Tappan Zee Hudson River Crossing, SR-182 Columbia River Bridge, the Thaddeus Kosciusko Bridge (I-87) (see Figure 1-1), the Allegheny River Bridge, PA, which carries I-76, and Fred Hartman Bridge, TX (see Figure 1-2). The structures located outside of the US include: Stonecutters Bridge and Ting Kau Bridge in Hong Kong, Zhejiang Xihoumen Bridge, Haihe Bridge in China, and Yi Sun-sin Bridge in Korea.

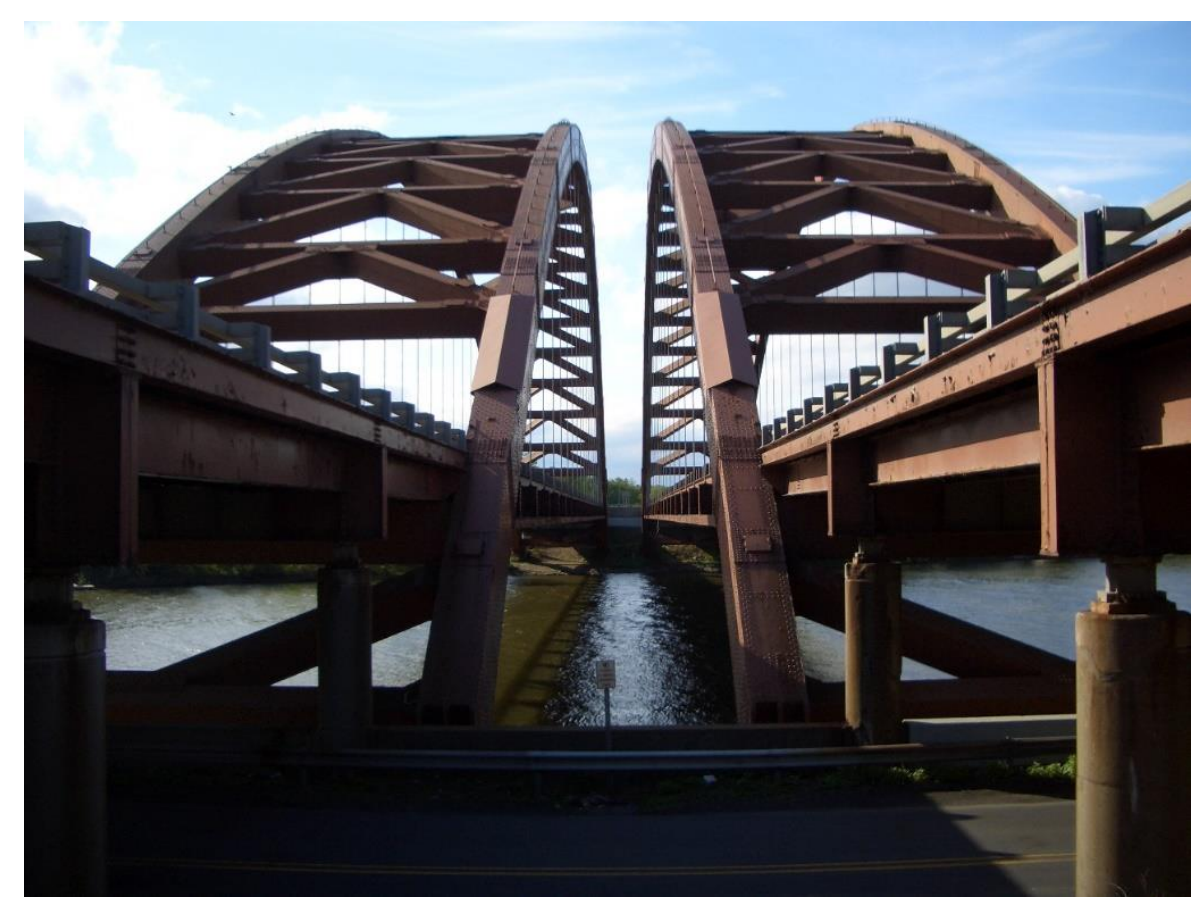

Figure 1-1: Thaddeus Kosciusko Bridge, NY, by Njhepler at English Wikipedia, CC BY 2.5, https://commons.wikimedia.org/w/index.php?curid=12452015 


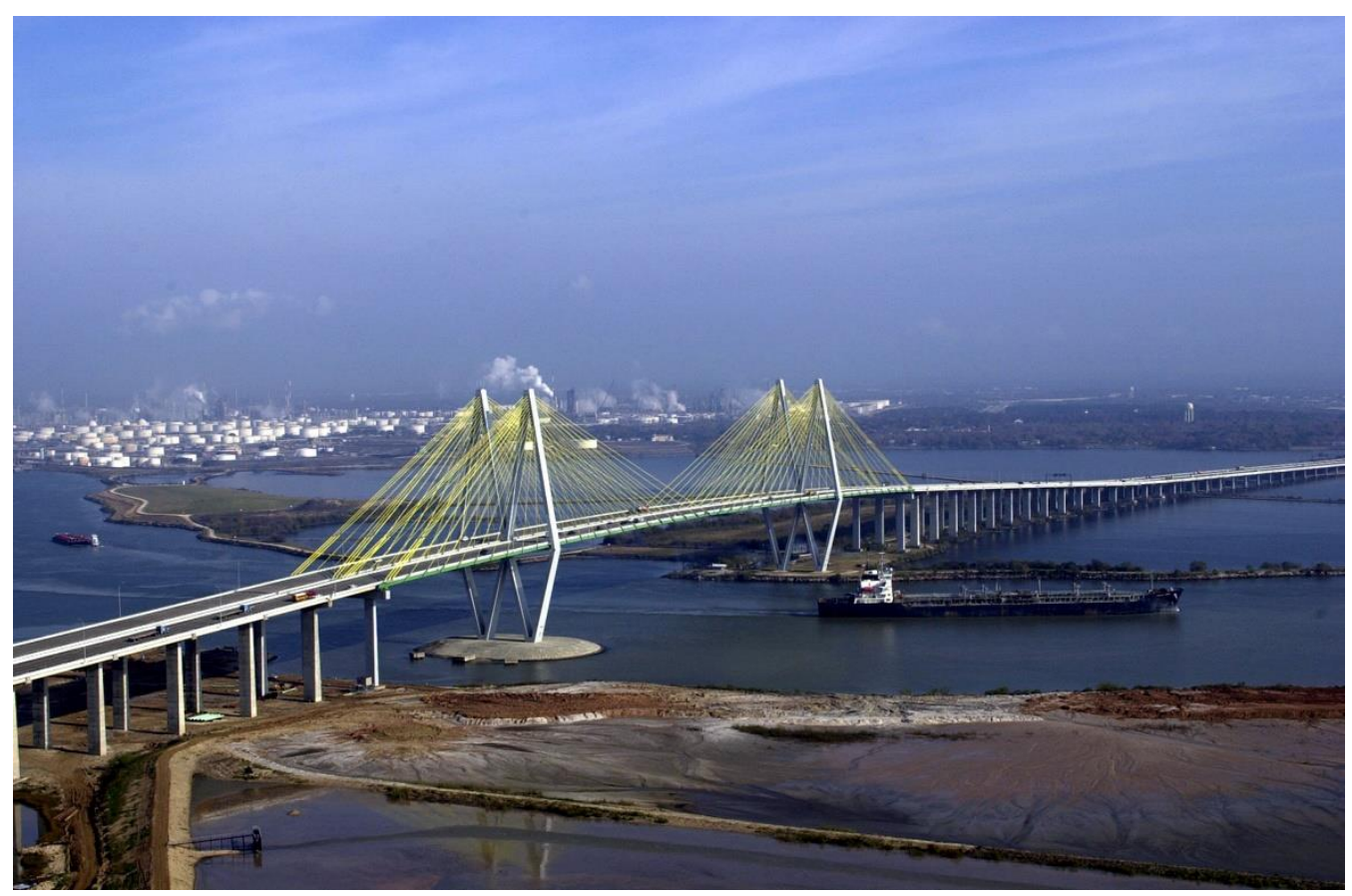

Figure 1-2: Fred Hartman Bridge, TX, by United States Coast Guard, PA2 James Dillard - U.S. Coast Guard Visual Information GalleryU.S. Coast Guard Visual Information Gallery Home, Public Domain, https://commons.wikimedia.org/w/index.php?curid=3499375

The experimental studies mostly cover the topic of aerodynamic interference effect on aerostatic coefficients. A set of tests at different Reynolds number is usually performed and the coefficients obtained for a twin deck are compared to those of a single deck. The main design parameter considered is the central gap width (see: [1], [2], [3], [4]). The results of static tests show that the upstream box is not significantly affected and behaves similarly to a single deck, as shown in [1] and [2]. In contrast, the pressure acting on the downstream box changes due to the characteristics of the slot. The other design parameter is the geometry of the bridge girders (see: [1], [3], [5]). The researchers analyze sets of chosen cross-sections to find the one with the most favorable aerodynamic characteristics and try to optimize an existing shape. The flow pattern, the vortexshedding frequency, and Strouhal number are also of interest, see [3], [4], [6], [7], [8], [9], [10]. The measurements reveal that as the gap ratio increases, the turbulent kinetic energy in the gap region and the fluctuating pressures on the downstream deck increase. Results show that, at certain flow velocities, the vortex induced vibrations of the upstream deck tend to be amplified due to the interference effect between parallel decks, see [8].

The literature review delivers a discussion on, other than gap width, modifications of the crosssection geometry, which would improve the aerodynamic properties. The proposed control measures include: bracings, wind fairings, stabilizing plates, and spoiler gratings fixed on the railing, among others, as presented in papers [5], [8], [11], [12], and [13]. 
CFD modeling compliments the wind tunnel testing and is used in parametric studies, where multiple cases need to be considered, like in [4]. 2D and 3D CFD URANS computations are performed to obtain aerostatic coefficients and to study the vortex shedding effect. CFD also helps to find an optimized cross-section shape and add modifications to the original design, e.g. adding wind barriers and sharpening the wind fairing noses of the two decks, (see [14], [15], [16], [17], [18], [19], [20]).

In this research, one of the existing twin-deck bridges, the Stonecutters Bridge in Hong Kong, is studied and used as a reference in the validation of the computational fluid dynamics analysis approach. Stonecutters Bridge is a cable-stayed bridge with a twin deck. Extensive studies, both experimental and computational, were performed on this bridge design in the past. Researchers concentrated on the investigation of the effects of gap-width and the angle of wind incidence on the aerodynamic characteristics of the bridge. Experimental measurements were presented in [1], [14] and [20]-[24], and the results of computational simulations are shown in [25] and [26], among others. Experimental tests were performed to assess the bridge model response to smooth and turbulent flow. Pressure distributions were studied to investigate the influence of the gap width in [1] and [3]. The analysis showed that the gap width significantly affects the pressure distribution on the decks and therefore their aerodynamic performance. Vortex shedding mechanisms of the bridge deck were also studied. They revealed that the bridge is susceptible to vortex shedding and that increasing the gap width causes the Strouhal number to increase. Dynamic tests of the twin-deck bridge model were conducted [21] to analyze the influence of the central gap. A comparative analysis showed that increasing the gap causes a higher torsional damping ratio, which increases the aerodynamic stability of the bridge. A detailed CFD study of flutter derivatives was performed in [26] at the in-service stage of the Stonecutters Bridge as well as during construction and the results indicate stability of the bridge. The adopted approach is validated by an analysis of aerodynamic behavior of the Stonecutters Bridge. The computational results are compared to experimental measurements found in literature.

\subsection{Proposed analysis methods}

This study was performed on a generic cross-section segment of a twin bridge deck model in an unsteady air flow. The analysis methods cover preliminary simulations, such as selection between Reynolds Averaged Navier-Stokes (RANS) equations with different turbulence models versus Large Eddy Simulations (LES), and a mesh density study. The main purpose of the research is to: characterize static and dynamic responses of the model twin decks by computing steady state aerodynamic forces and pitching moment in RANS simulations as well as computing the time history of aerodynamic forces and pitching moment obtained from LES simulations. The research also seeks to characterize the influence of the upstream deck wake on the downstream deck. Static simulations include a parametric study with various angles of wind incidence, different wind speed values, and a study of gap-to-width ratios. 


\section{CFD model}

\subsection{General information}

The CFD model is set up to represent wind tunnel experiments. The domain geometry consists of three parts: the wind tunnel nozzle, trapezoidal transition part, and the main cube modeling the room. The dimensions are such that the boundary conditions do not have a significant influence on the flow around the bridge model. The inlet is far enough from the bridge model for the flow to be developed in front of it and also the domain is long enough that there is no influence of reverse flow that may occur near the pressure outlet. All outer surfaces of the model, excluding the inlet and outlet surfaces, have a no-slip wall boundary condition applied.

The CFD model with a twin deck test section inside is presented in Figure 2-1. The deck dimensions are as follows: deck width (B) - $0.381 \mathrm{~m}$, deck thickness - $0.022 \mathrm{~m}$, girder height $0.031 \mathrm{~m}$, length $-1.524 \mathrm{~m}$. In the study of twin deck models, four gap widths (L) are considered: $0.05 \mathrm{~m}, 0.1 \mathrm{~m}, 0.2 \mathrm{~m}, 0.3 \mathrm{~m}$, which correspond to the gap to single deck width (L/B) ratio of 0.13 , $0.26,0.52$, and 0.79. An example mesh around the decks is presented in Figure 2-2. The mesh is denser around the decks and the wake area to capture the character of the flow and the interference between the decks. Although the research is focused on the flow around twin-deck bridges, a set of runs is performed with a single deck as well, for comparison purposes.
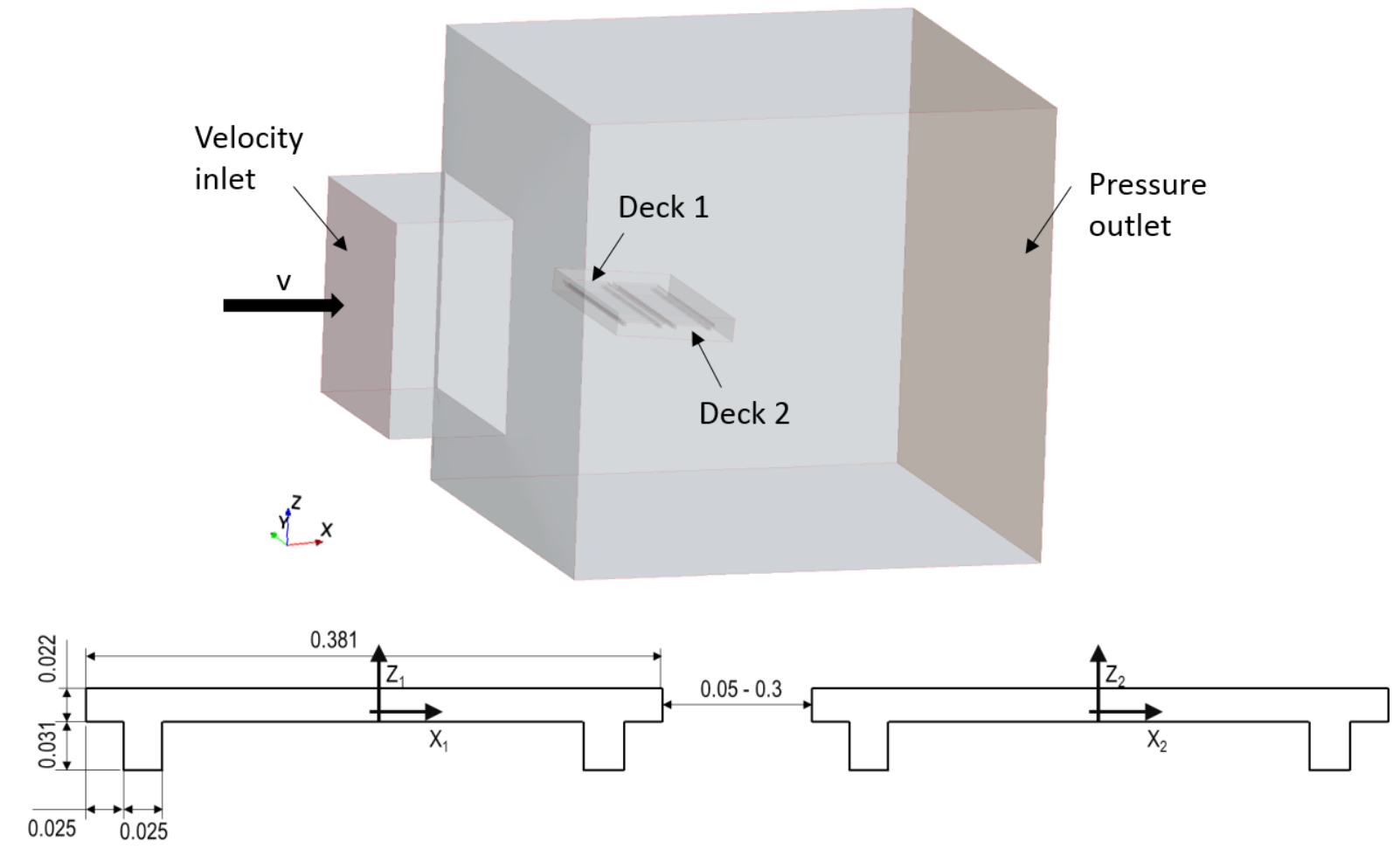

Figure 2-1: The computational domain with twin deck dimensions in meters and locations of local CSYS origins 


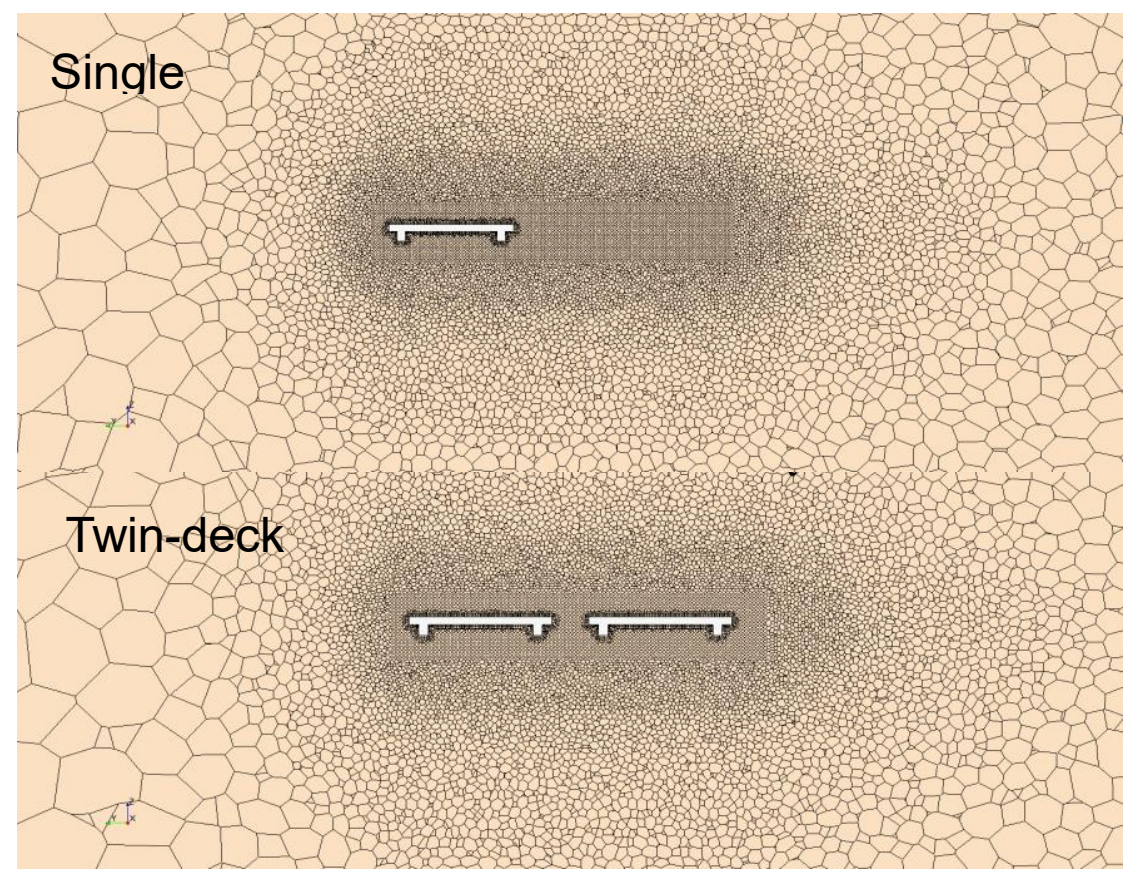

Figure 2-2: An example mesh around single and twin deck cross sections

\subsection{Mesh study}

The influence of mesh density on the results is investigated in the first step of this study. A model of a twin deck bridge with a $0.1 \mathrm{~m}$ gap (gap ratio=0.26) was used. In all cases a prism layer with constant thickness is created around the decks to provide layers of prism cells next to the wall. The 'all Y+ treatment' option is used, which uses a blended wall function to estimate shear stress if $5<\mathrm{Y}+<30$ and the standard wall function for $\mathrm{Y}+>30$. Two prism layer stretching values are considered. The first stretching value is 1.0, with 1, 2, 4, 8 and 16 layers (which gives $\mathrm{Y}+=40,21$, 9.4, 3.7 and 2.1 accordingly). The second stretching value is 1.5, with 8 layers (which gives $\mathrm{Y}+=1.3$ ). The number of volume cells varies from approximately 3 to 4 million cells.

Two types of meshing techniques were used. The polyhedral mesher is suited to complex, multiregion geometries. These cells usually have 12 to 14 faces. The trimmer mesher uses predominantly hexahedral mesh cells with trimmed cells next to surfaces. Table 2-1 shows which meshing techniques were used in the simulations, along with a close-up view of the resulting prism layer around a corner of the deck. 
Table 2-1. Types of meshes used in the simulations

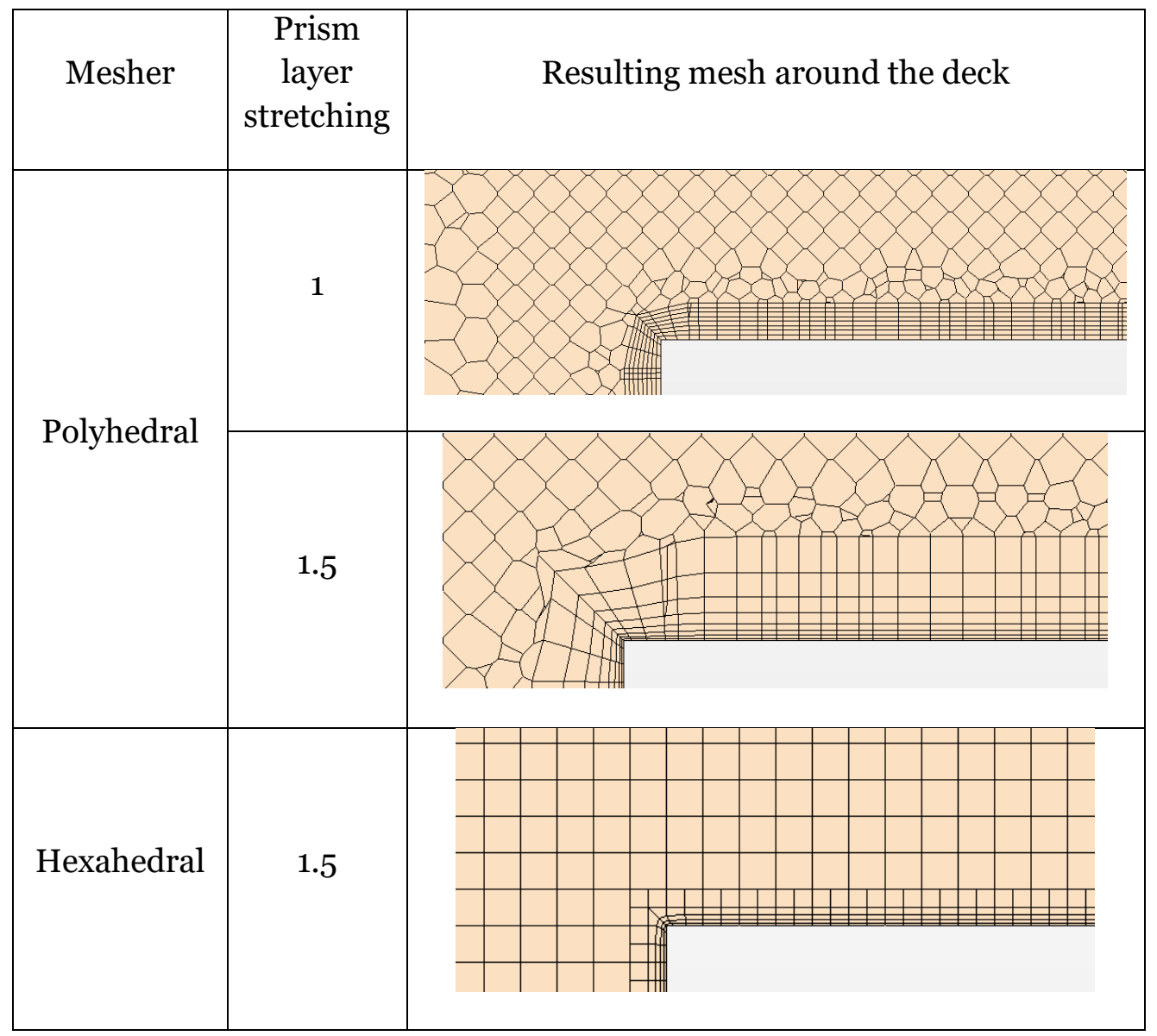

An unsteady RANS (URANS) solver was used in the computations as well as the Large Eddy Simulation model. The simulations were kept running until the flow reached a steady state and force and moment components on the stationary decks converged to a constant value.

Computations using the URANS solver with the k- $\varepsilon$ turbulence model and polyhedral mesh were performed for different thicknesses of the first prism layer. Moreover, for the densest mesh (and thinnest first layer) LES computations were done using both polyhedral and trimmer meshers. Plots of the computed drag and lift force are presented in Figure 2-3 and Figure 2-4. The parametric mesh study shows that the biggest differences in drag and lift forces for both decks occur between the lowest $(\mathrm{Y}+=1)$ and highest values of $\mathrm{Y}+(\mathrm{Y}+=40)$. Two highest values of $\mathrm{Y}+$ give very close values for drag and lift force components, but not for pitch moment on the upstream deck. For the instance of the lowest $\mathrm{Y}_{+}$, most simulations give very similar results except for LES with hexahedral mesh. 


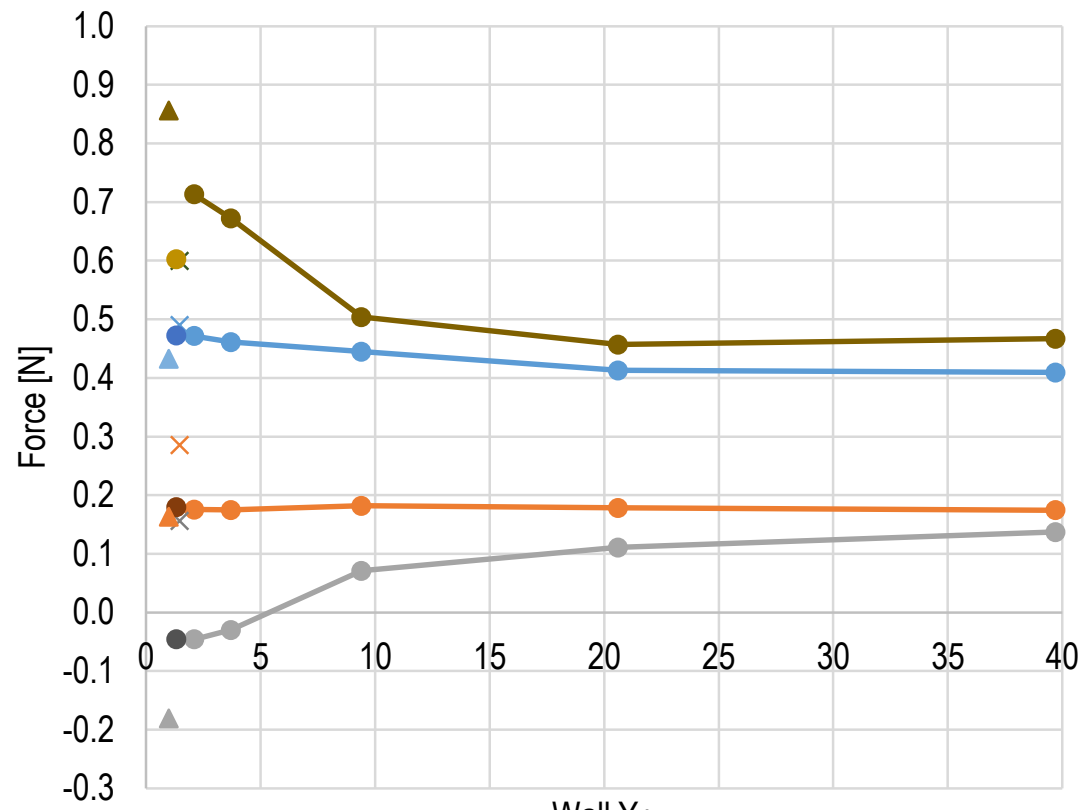

Wall Y+

\begin{tabular}{|c|c|}
\hline$\rightarrow$ Drag force - deck 1 & $\rightarrow$ Lift force- deck 1 \\
\hline- Drag force- deck 2 & $\rightarrow$ Lift force - deck 2 \\
\hline$\times$ Drag force - deck 1 (LES P) & $\times \quad$ Lift force - deck 1 (LES P) \\
\hline$\times$ Drag force - deck 2 (LES P) & $\times \quad$ Lift force - deck 2 (LES P) \\
\hline - Drag force - deck 1 (ratio=1.5) & Lift force - deck 1 (ratio=1.5) \\
\hline - Drag force - deck 2 (ratio=1.5) & - Lift force - deck 2 (ratio=1.5) \\
\hline$\Delta$ Drag force - deck 1 (LES H) & - Lift force - deck 1 (LES H) \\
\hline$\Delta$ Drag force - deck 2 (LES H) & $\Delta \quad$ Lift force - deck 2 (LES H) \\
\hline
\end{tabular}

Figure 2-3: Force component values vs. wall $\mathrm{Y}+(\mathrm{P}-$ polyhedral mesh, $\mathrm{H}$ - hexahedral mesh, ratio - stretch factor for the cell thickness in the prism layer) 


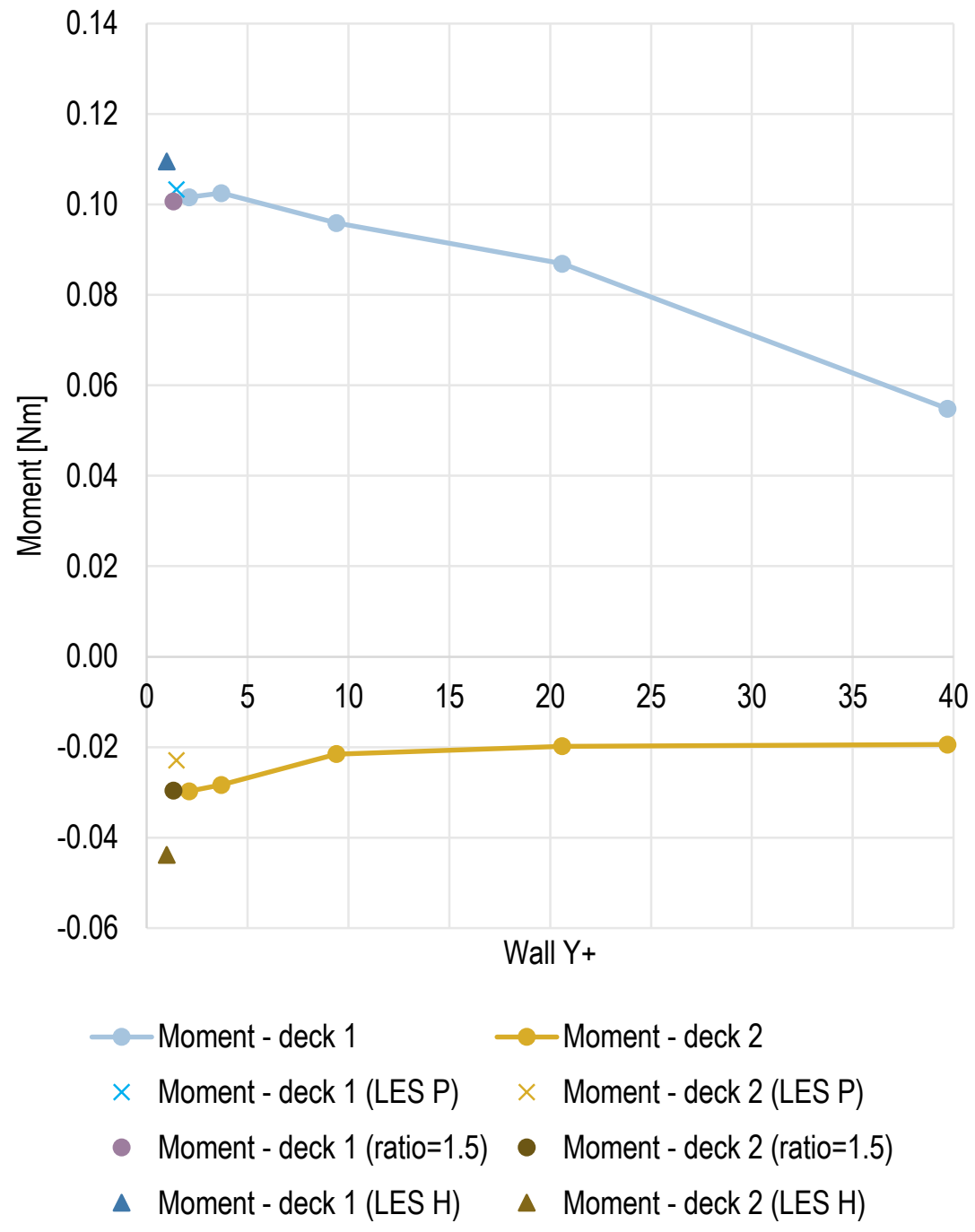

Figure 2-4: Pitch moment component values vs. $\mathrm{Y}+(\mathrm{P}$ - polyhedral mesh, $\mathrm{H}$ - hexahedral mesh, ratio - stretch factor for the cell thickness in the prism layer)

The influences of mesh type and mesh density on the force components are also shown in Figure 2-5 and Figure 2-6 in the form of histograms. The first two columns refer to polyhedral and hexahedral mesh with $\mathrm{Y}+=20$, the next two columns refer to polyhedral and hexahedral mesh with $\mathrm{Y}+=1$, and the last column refers to LES simulations with polyhedral mesh. 
(a)

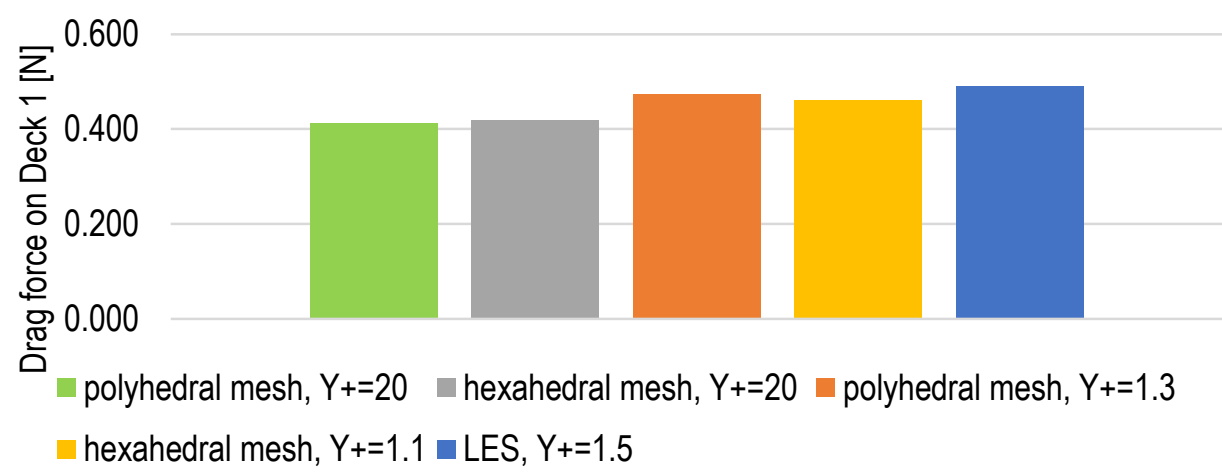

(b)

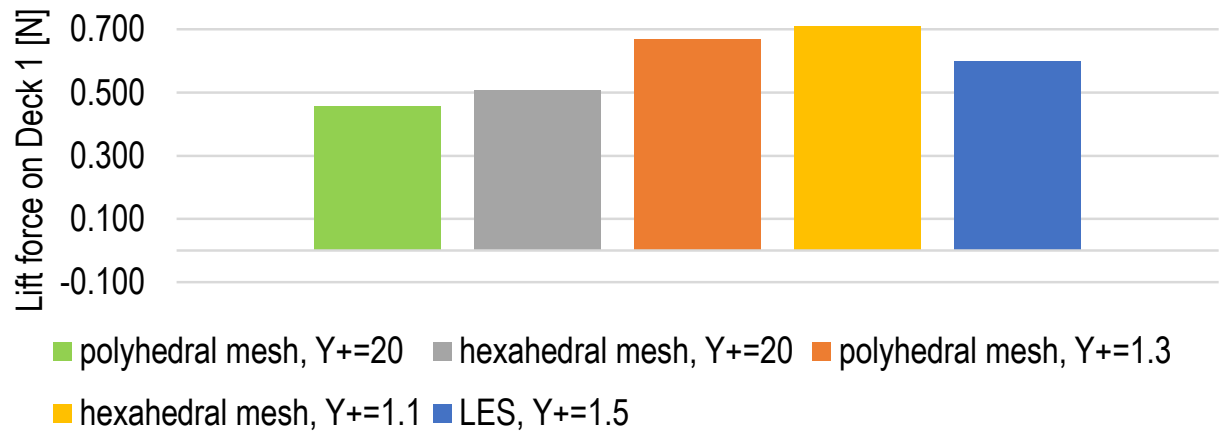

(c)

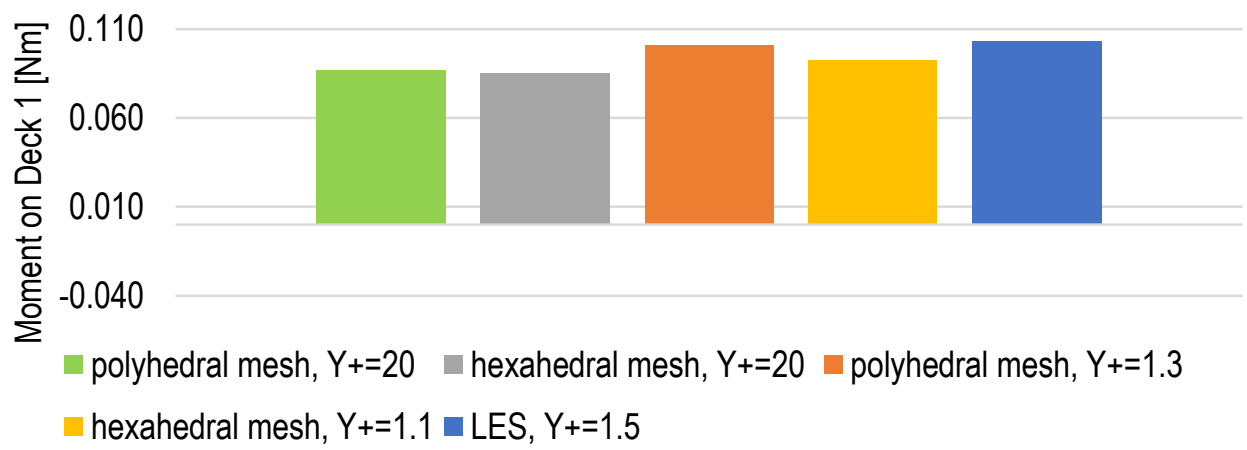

Figure 2-5: Mesh influence on force and moment components values acting on the upstream deck

(a)

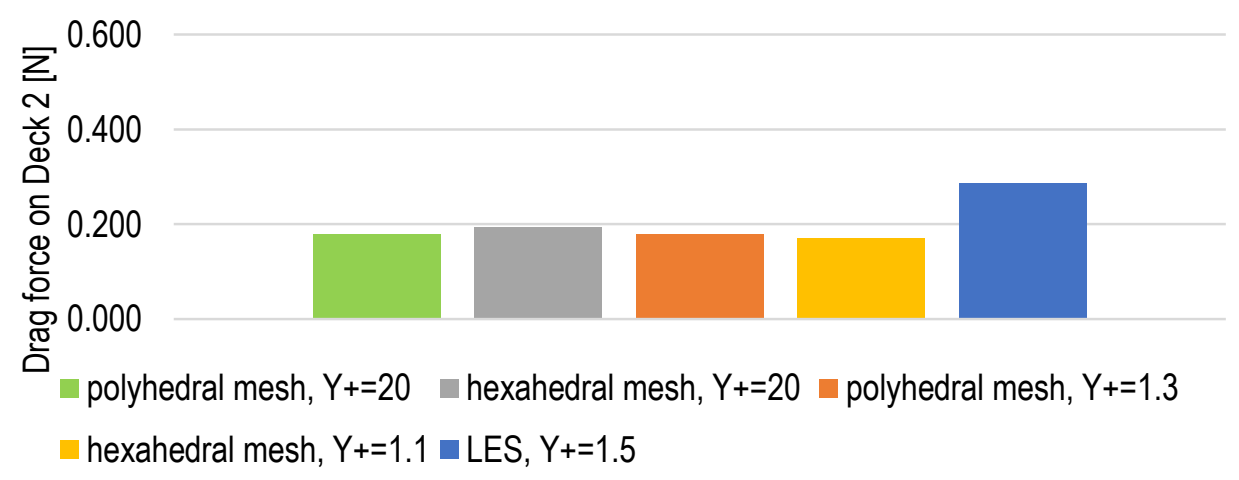

Investigation of Aerodynamic Interference between Twin Deck Bridges 
(b)

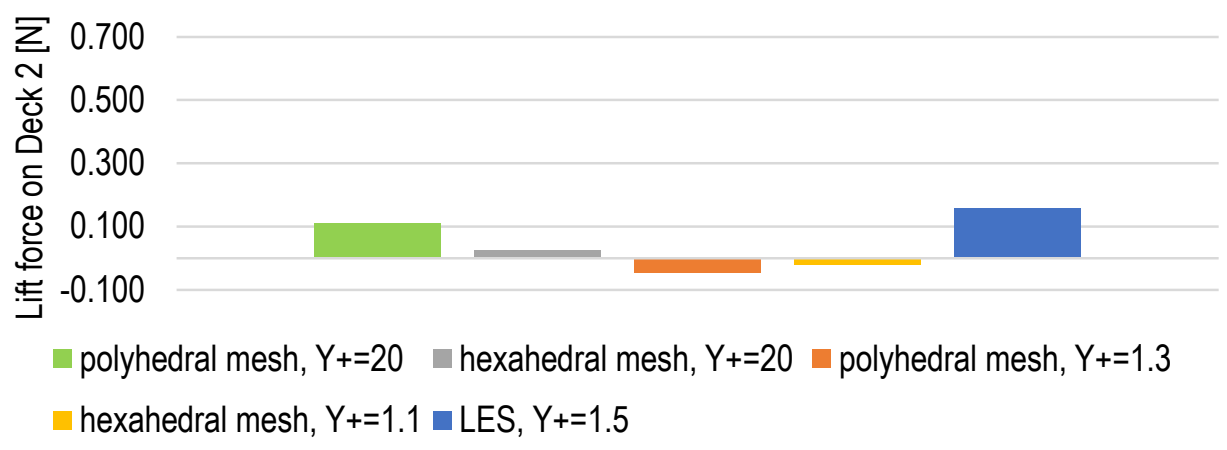

(c)

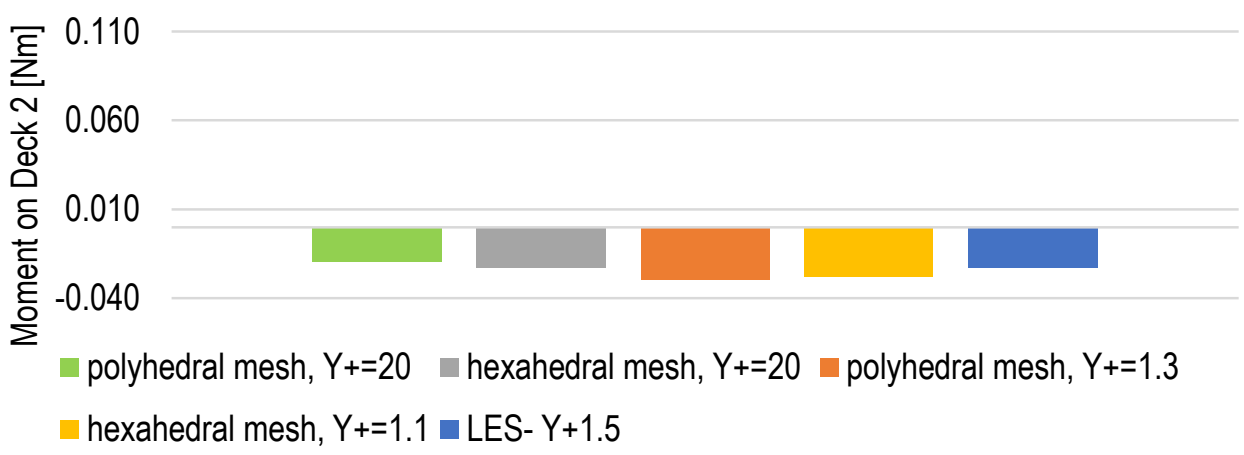

Figure 2-6: Mesh influence on force and moment components values acting on the downstream deck

\section{Analysis of the flow around a single deck bridge}

A set of runs was performed to assess the character of the flow around a single deck. The results presented in this chapter are used as a reference for the twin deck study.

The pressure and velocity contour plots for a single deck in a wind tunnel are shown in Figure 3-1 and Figure 3-2. The zoomed out view shows the pressure distribution relative to the atmospheric reference pressure and the velocity magnitude in a cross section of the domain. The pressure is nearly uniform away from the deck, and the flow separation and wake are visible in the velocity magnitude color plot. The close-up view of the flow around the deck makes it possible to see the distributions around the deck in greater detail. Pressure reaches the highest values on the upstream side - vertical and bottom surfaces where the approach flow stagnates. The lowest (negative in value) pressure is located on the top surface, right at the front. Moreover, multiple separation points can be seen in the velocity contour plot as the deck cross-section is not streamlined. 
(a)

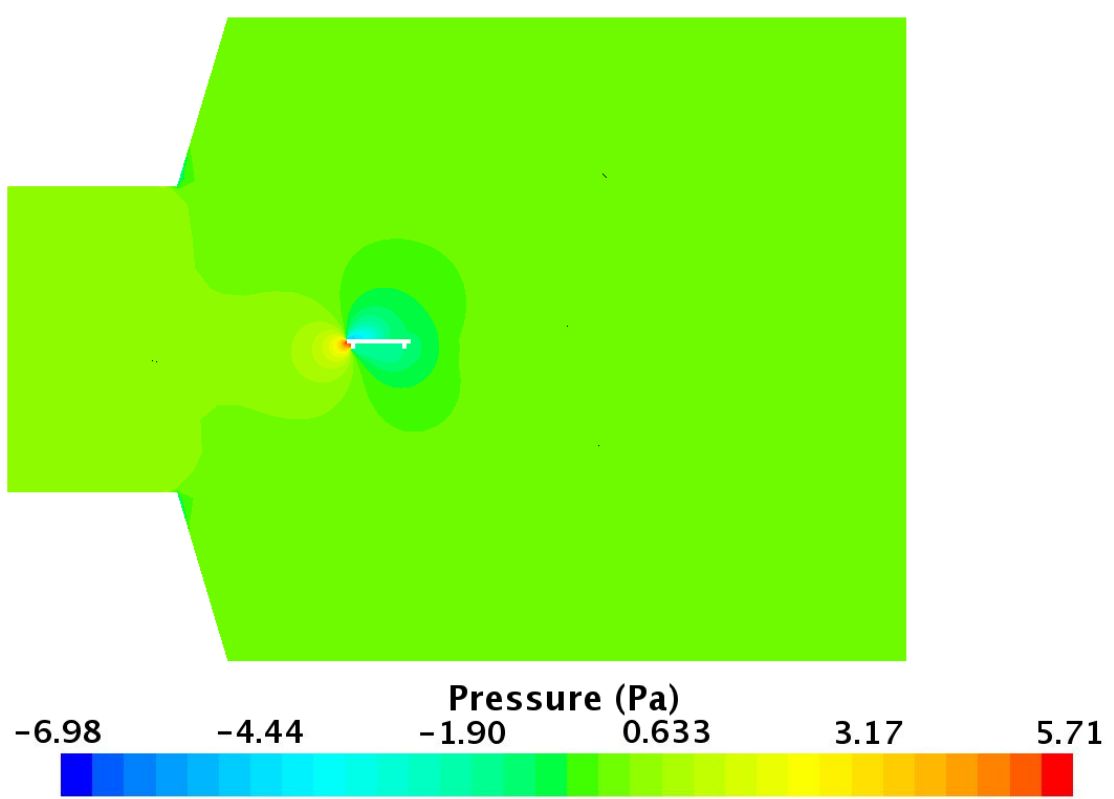

(b)

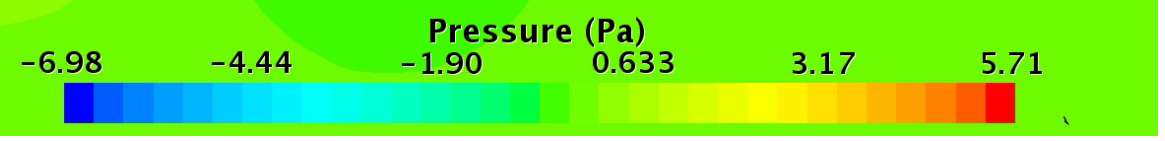

Figure 3-1: Single deck. Pressure plot on the mid section of the domain (a) zoomed out view, (b) the area around the deck 
(a)

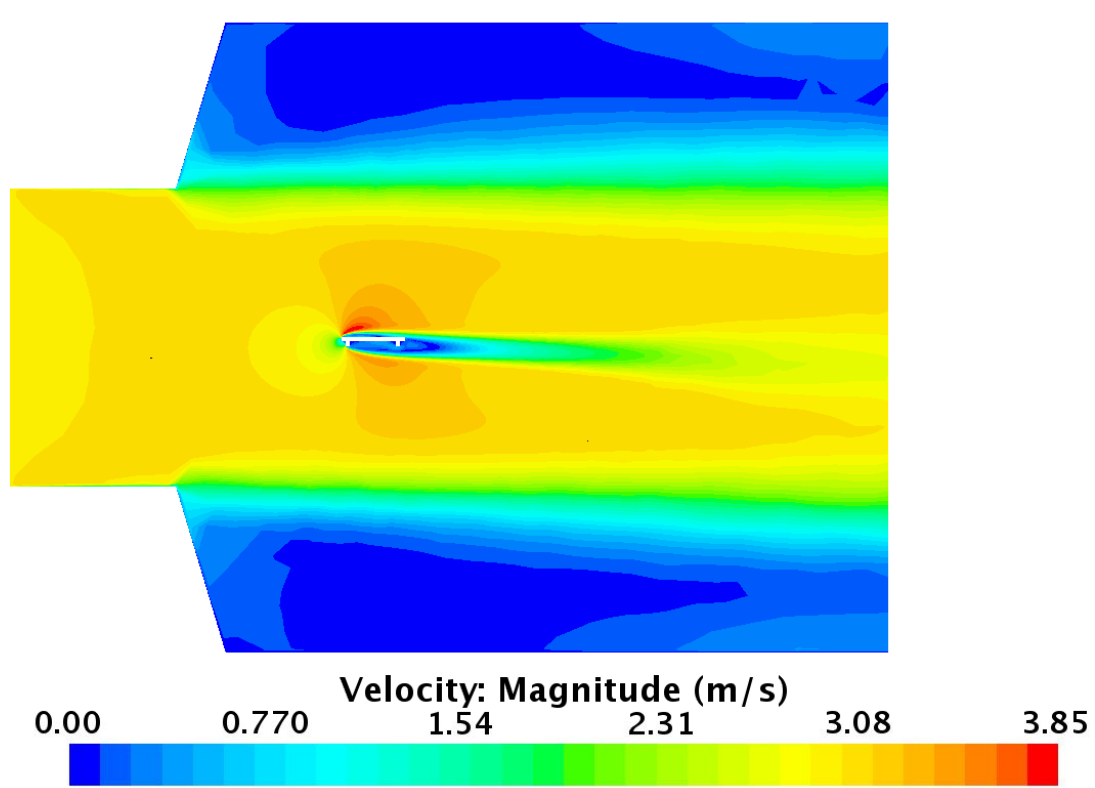

(b)

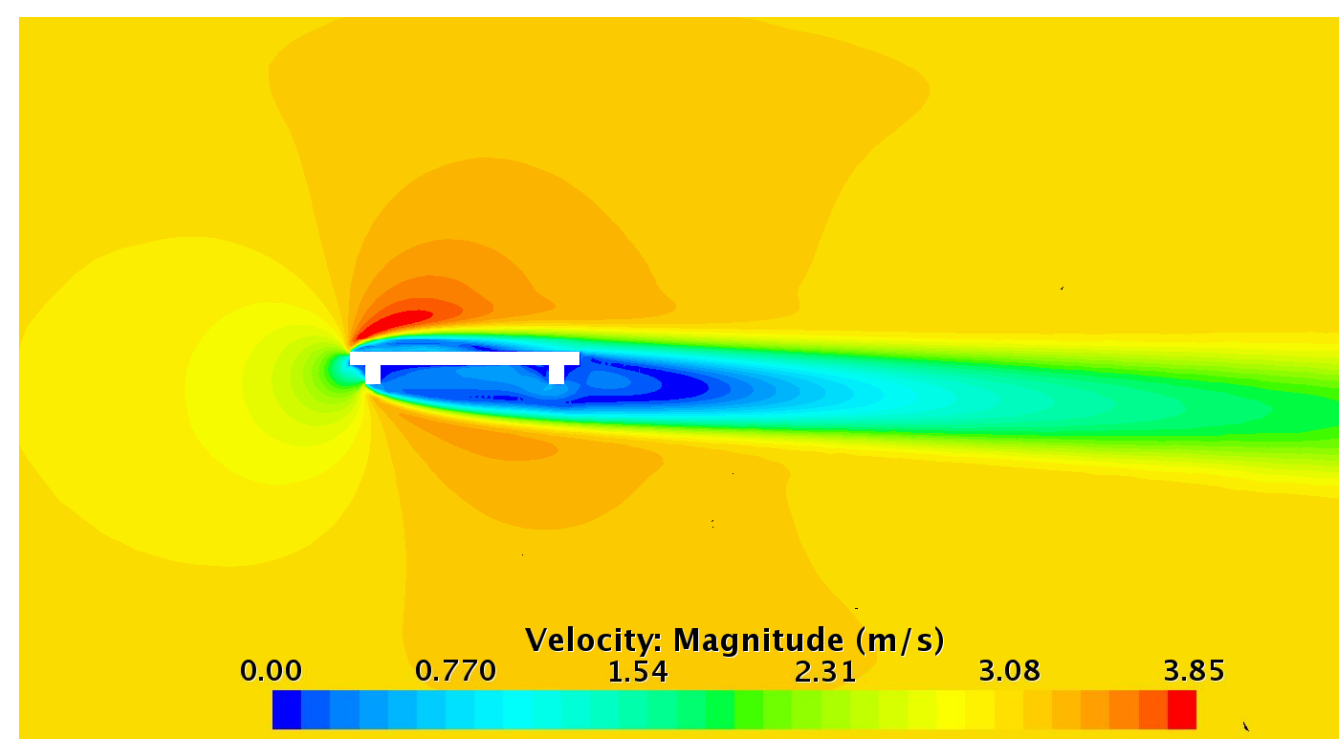

Figure 3-2: Single deck. Horizontal velocity plot on the mid section of the domain (a) general view, (b) the area around the deck

The pressure distribution around the deck was checked for different flow directions. Angles of attack in the range of -10 deg to 10 deg with a 5 deg interval were selected. The resultant pressure fields on the center cross section of the deck are collected in Table 3-1. Table 3-2 summarizes velocity field contour plots. The extreme values of pressure and velocity for these cases are listed in Table 3-3. The minimum pressure on the deck, equal to $-8.37 \mathrm{~Pa}$, is located at the top upstream corner of the deck and occurs when the angle of attack equals $5 \mathrm{deg}$. The highest positive pressure value, 5.7 Pa, is found on the upstream surface of the deck. The velocity field on the central plane changes with the angle of attack. It is smooth in the -5 deg to 5 deg range. For the angles $-10 \mathrm{deg}$ and 10 deg a more turbulent flow forms behind the deck. 
Table 3-1: Pressure field contour plots for a single deck bridge at varying angle of attack

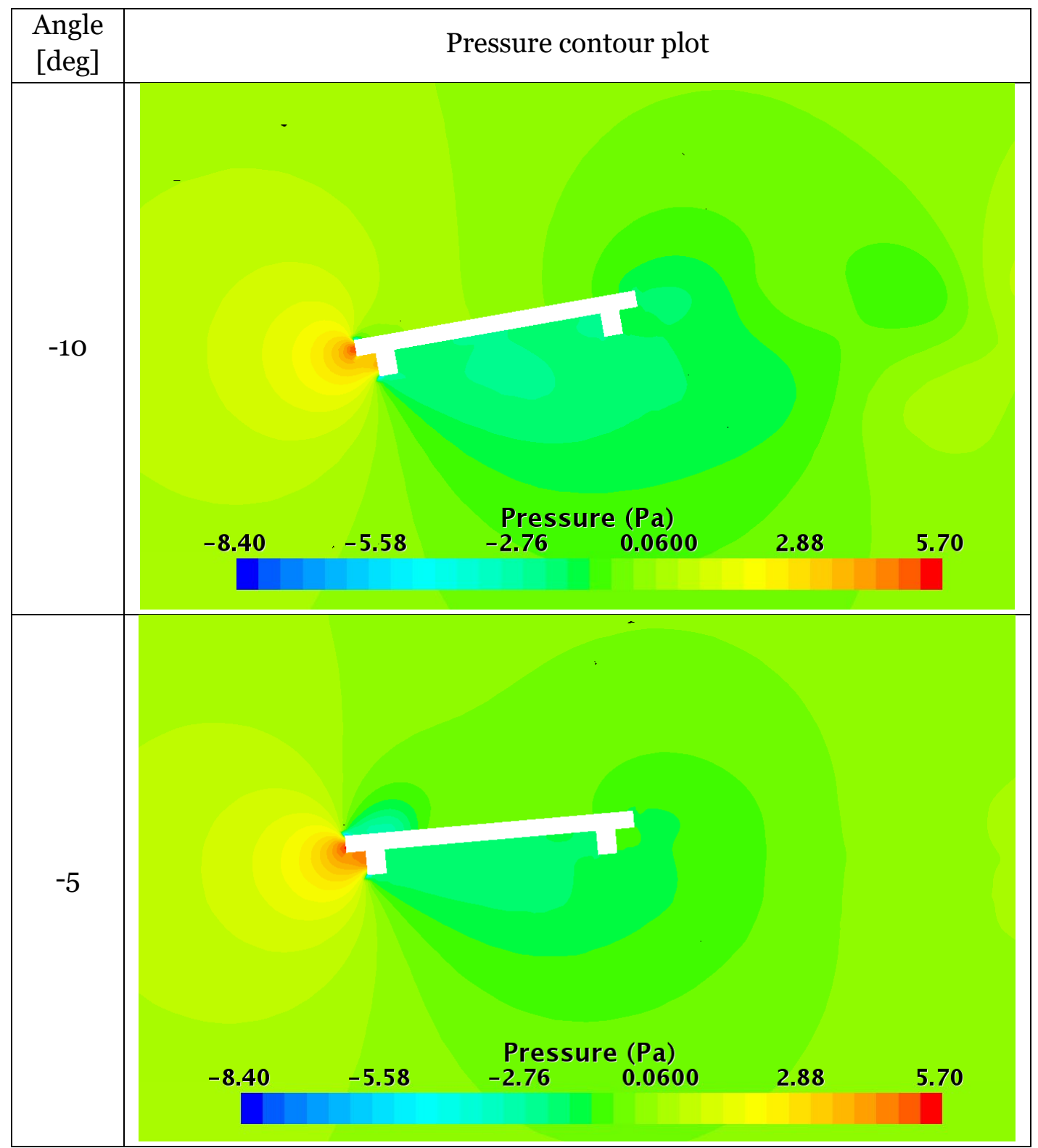




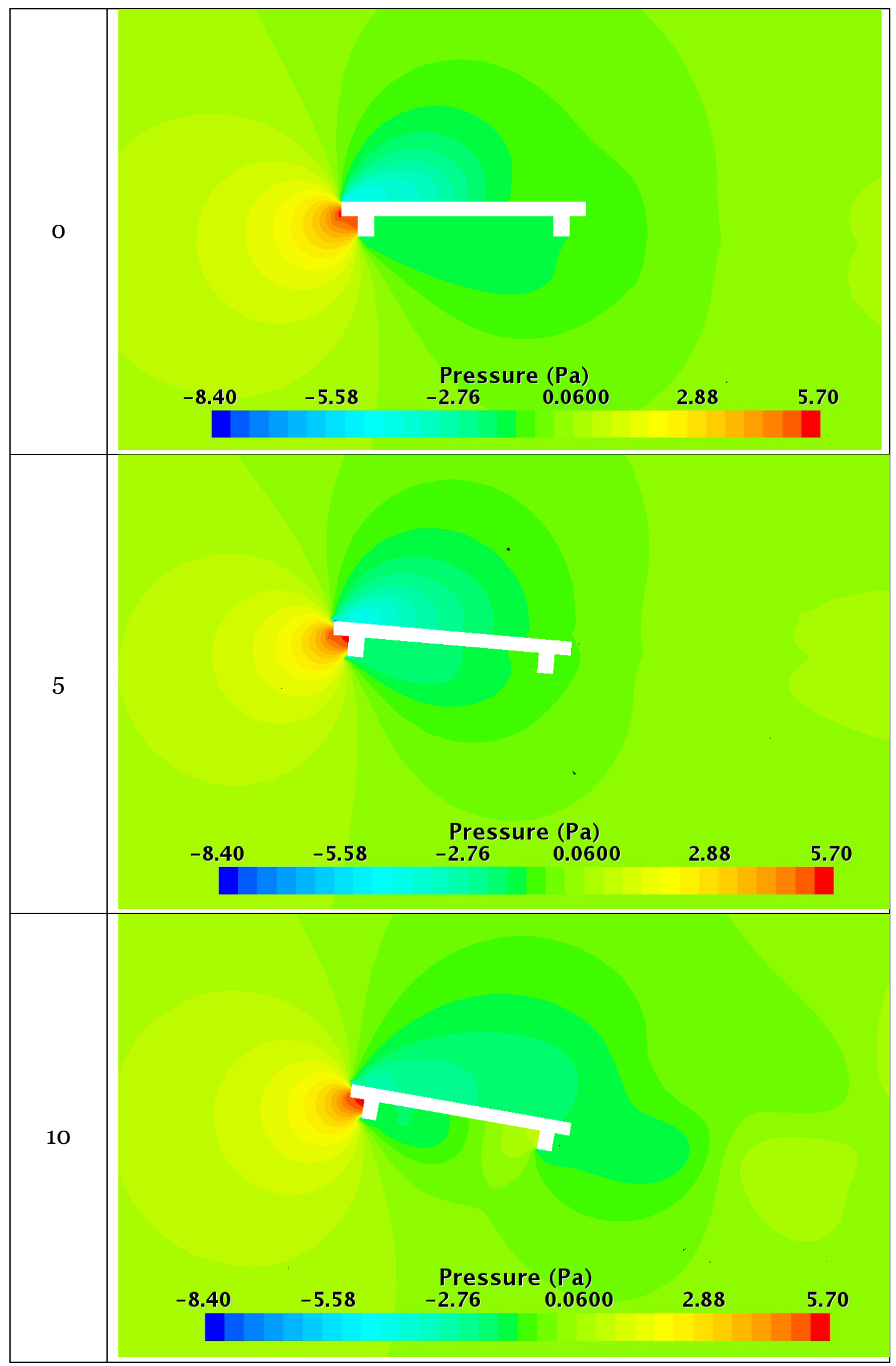

Investigation of Aerodynamic Interference between Twin Deck Bridges

Page | 14 
Table 3-2: Velocity field contour plots for a single deck bridge at varying angle of attack

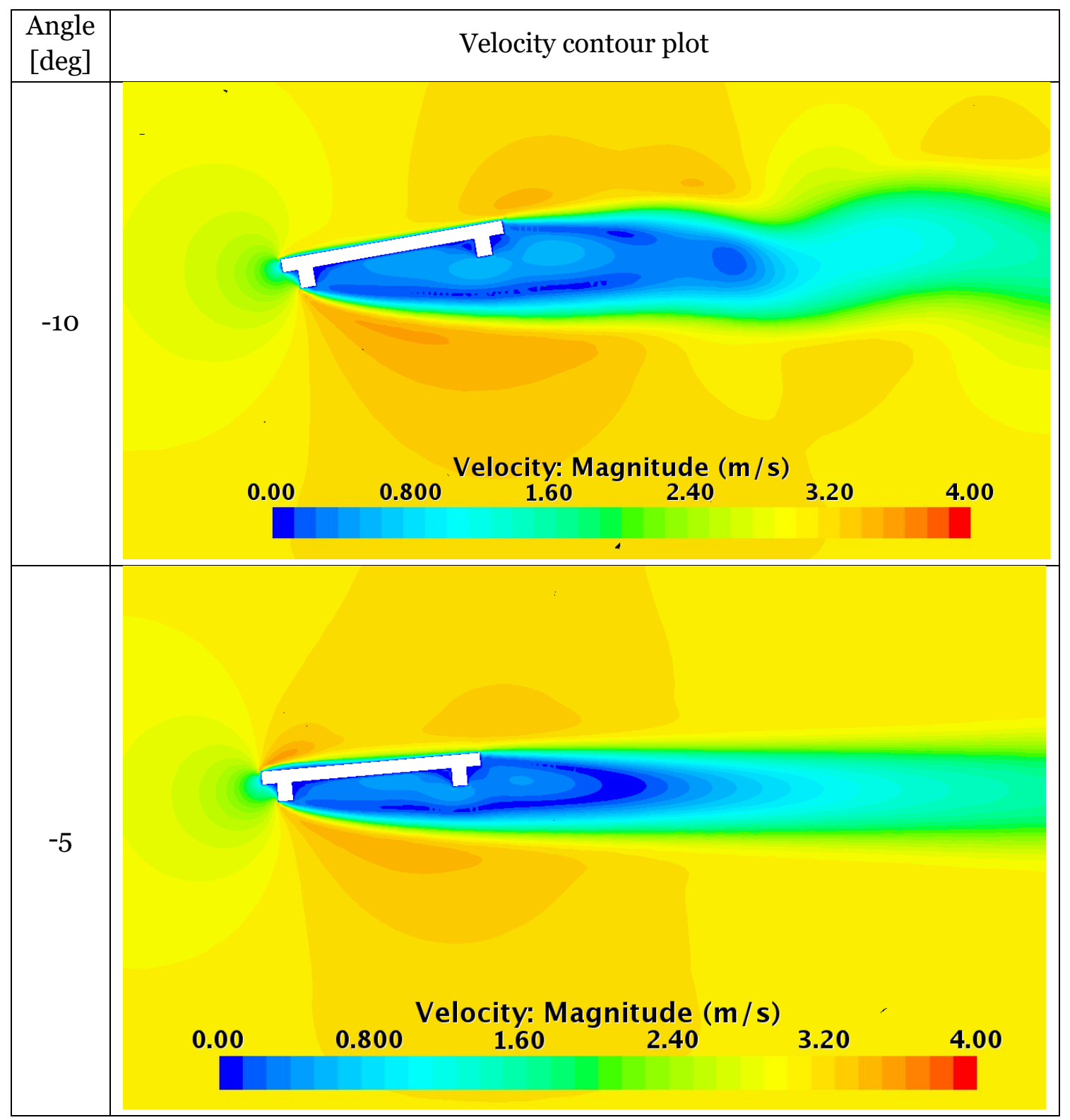




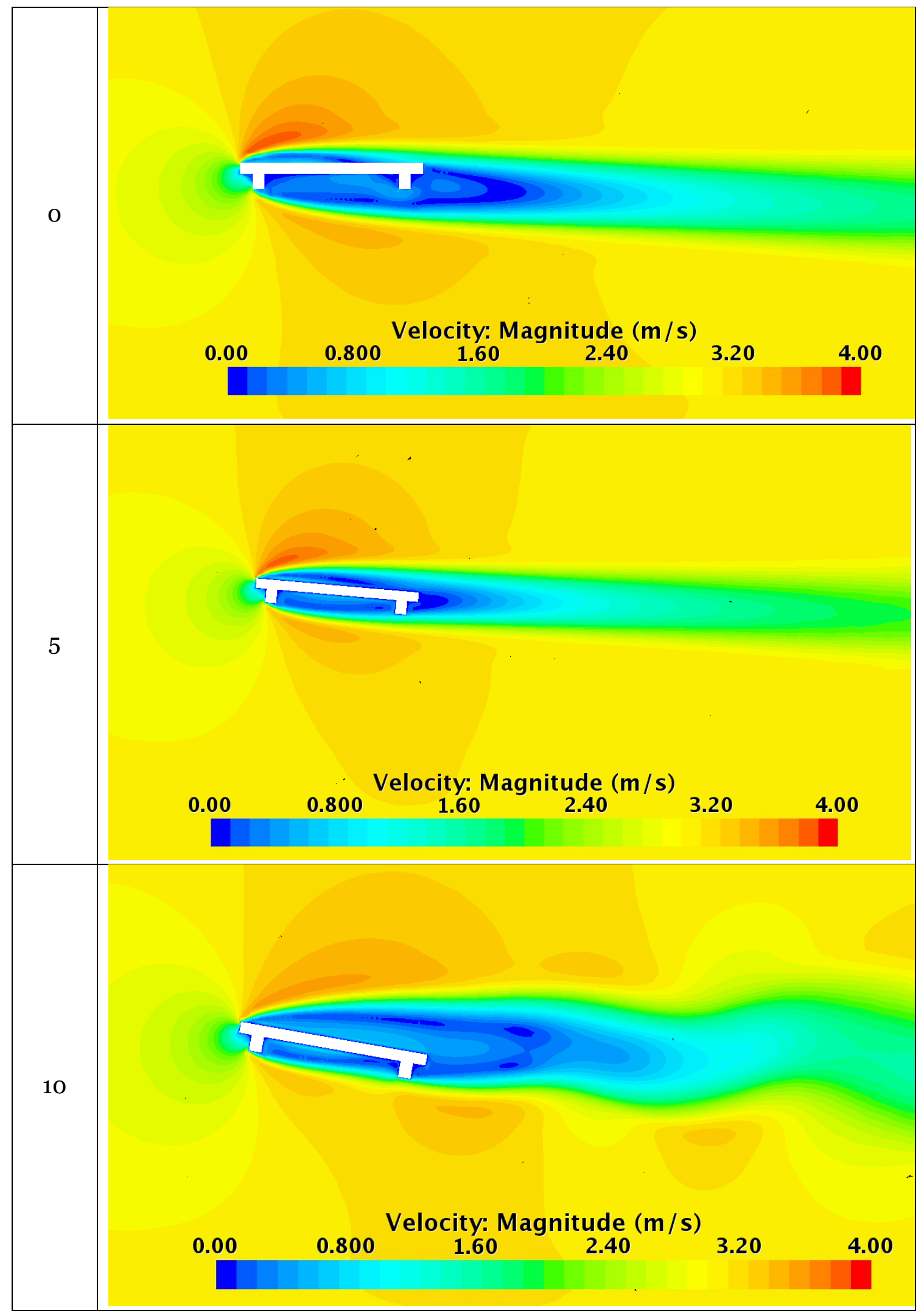

Investigation of Aerodynamic Interference between Twin Deck Bridges

Page | 16 
Table 3-3: Extreme values of pressure and velocity on a single deck

\begin{tabular}{|c|c|c|c|}
\hline Angle [deg] & \multicolumn{2}{|c|}{ Pressure [Pa] } & Velocity [m/s] \\
\hline & $\min$ & $\mathbf{m a x}$ & $\mathbf{m a x}$ \\
\hline-10 & -6.76 & 5.37 & 3.54 \\
\hline-5 & -7.51 & 5.48 & 3.58 \\
\hline 0 & -6.98 & 5.70 & 3.85 \\
\hline 5 & -8.37 & 5.34 & 3.86 \\
\hline 10 & -6.23 & 5.38 & 3.98 \\
\hline
\end{tabular}

\section{Analysis of the flow around a twin deck bridge}

\subsection{Pressure acting on a twin deck bridge}

Figure 4-1 illustrates pressure contour plots around twin deck cross-sections with a $0.1 \mathrm{~m}$ gap (gap ratio=0.26). The figures show a comparison of pressure fields for two models: URANS solver and LES with a low $\mathrm{Y}+$. A polyhedral mesh was used in all cases. The value ranges are kept constant for an easier comparison.

The pressure distribution around the upstream deck is very similar to the one observed around the single deck, described in Chapter 3. The downstream deck is located in its wake and the pressure acting on it is much lower. Figure 4-2 to Figure 4-9 provide detailed information about the pressure values at a set of points chosen on each surface of the model. The results presented for LES computations are time-averaged. The results obtained with the k- $\varepsilon$ model are very similar, regardless the mesh size at most of the points, except for the ones located in the corners. As the pressure gradients are high in these regions it is important to use a denser mesh, so that no information is lost. A comparison of two models with the same Y+ shows that they give a similar pressure distribution in the areas of the deck where the flow is less turbulent and does not have active vortex shedding. There are larger differences between the URANS and LES model results along the top of the upstream deck shown in Figure 4.2 (b) where there are passing vortices shed from the leading edge of the upstream deck. These differences show that the time averaged LES pressure values do not closely match the URANS results in a region of active vortex transport. 
(a)

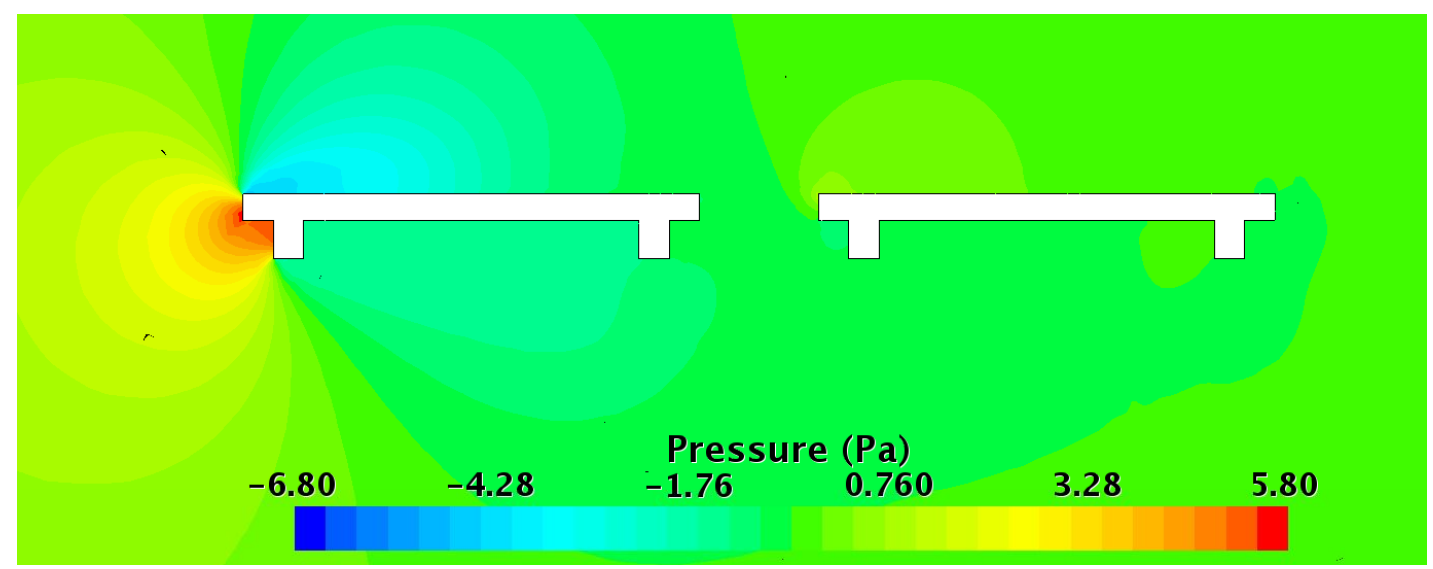

(b)

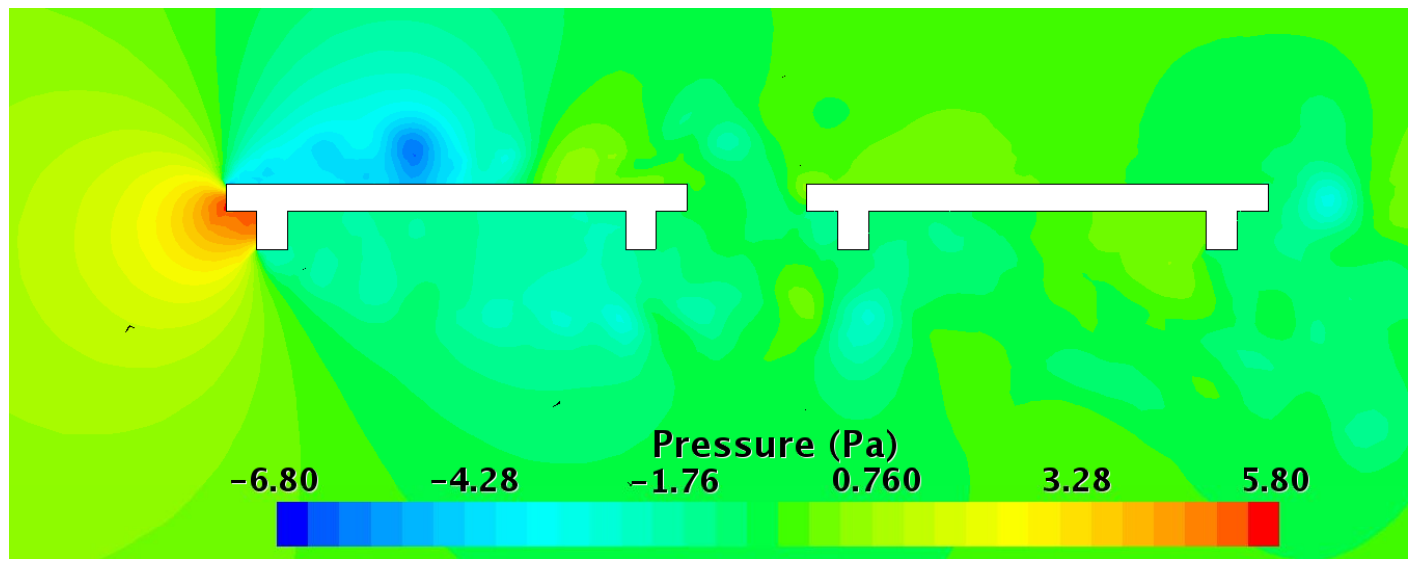

Figure 4-1: Pressure contour plot around the decks, (a) URANS, (b) LES at an instant in time, polyhedral mesh, $\mathrm{Y}+=1$ 
(a)

(b)
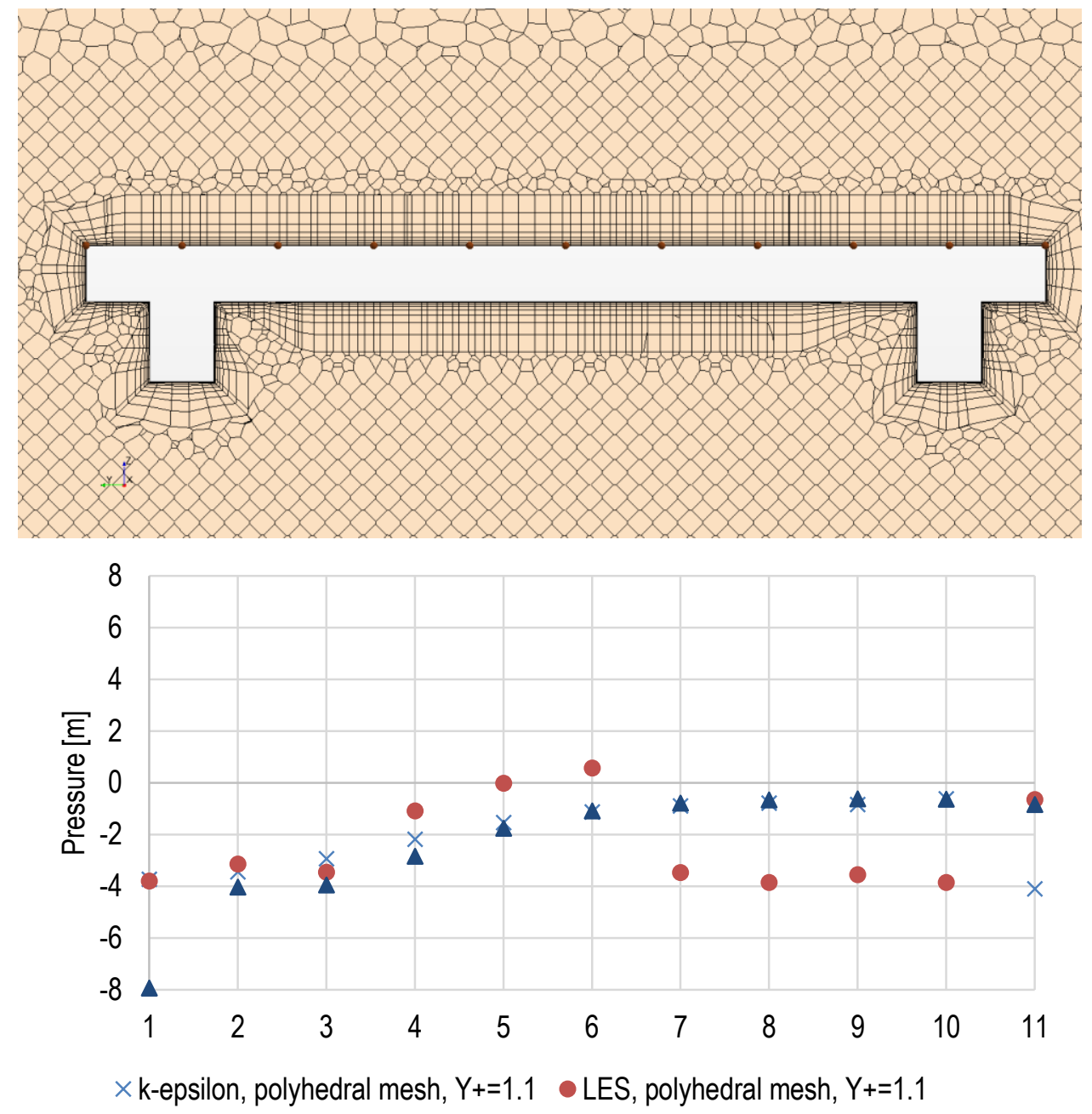

$\Delta$ k-epsilon, polyhedral mesh, $Y+=20.6$

Figure 4-2: Pressure on the symmetry line of the top surface of upstream deck, (a) location of measured points, (b) pressure values 
(a)

(b)
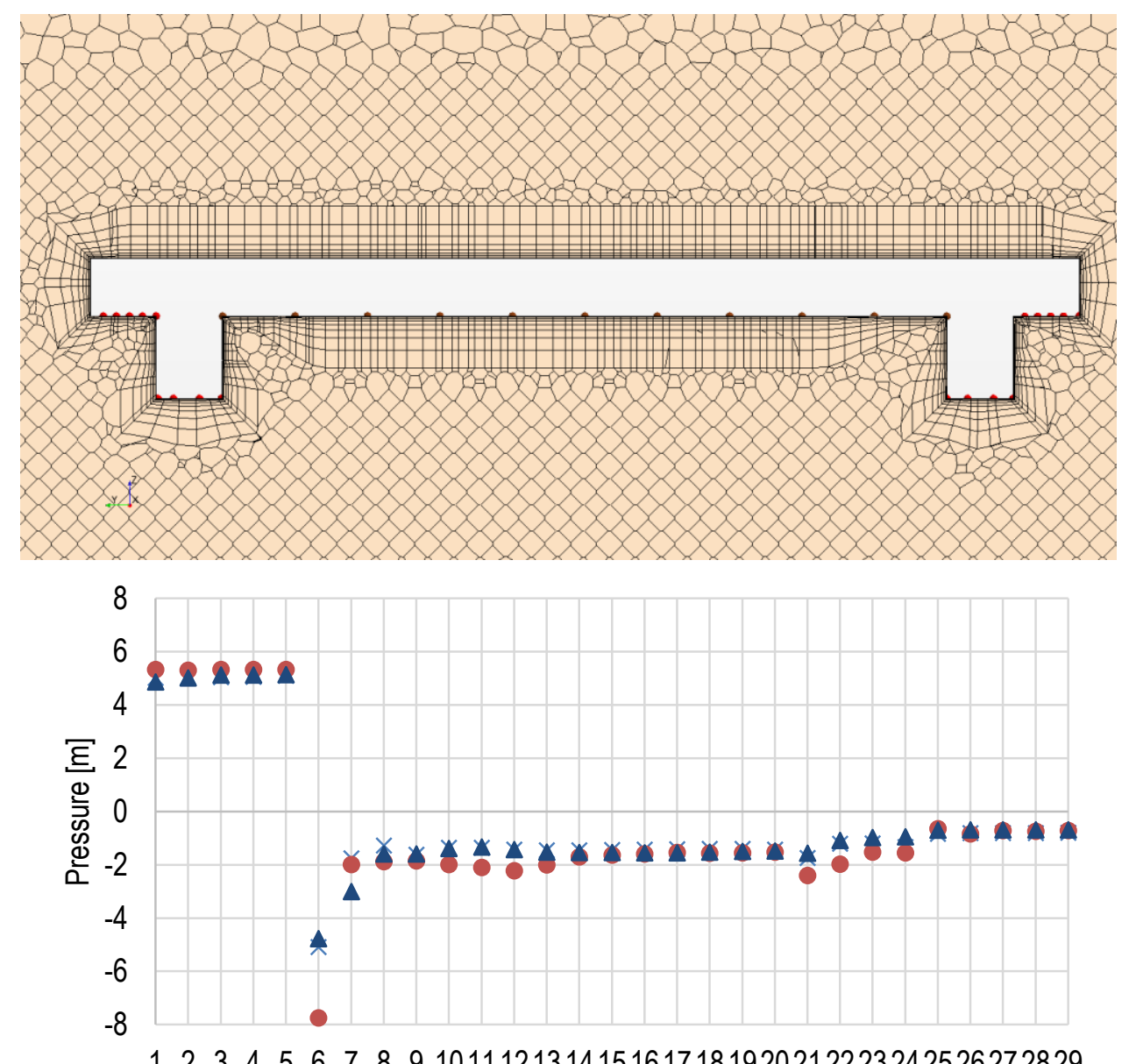

1234567891011121314151617181920212223242526272829

$\times$ k-epsilon, polyhedral mesh, $Y_{+}=1.1 \quad$ LES, polyhedral mesh, $Y_{+}=1.1$

$\Delta$ k-epsilon, polyhedral mesh, $Y+=20.6$

Figure 4-3: Pressure on the symmetry line of the bottom surface of the upstream deck, (a) location of measured points, (b) pressure values 
(a)

(b)
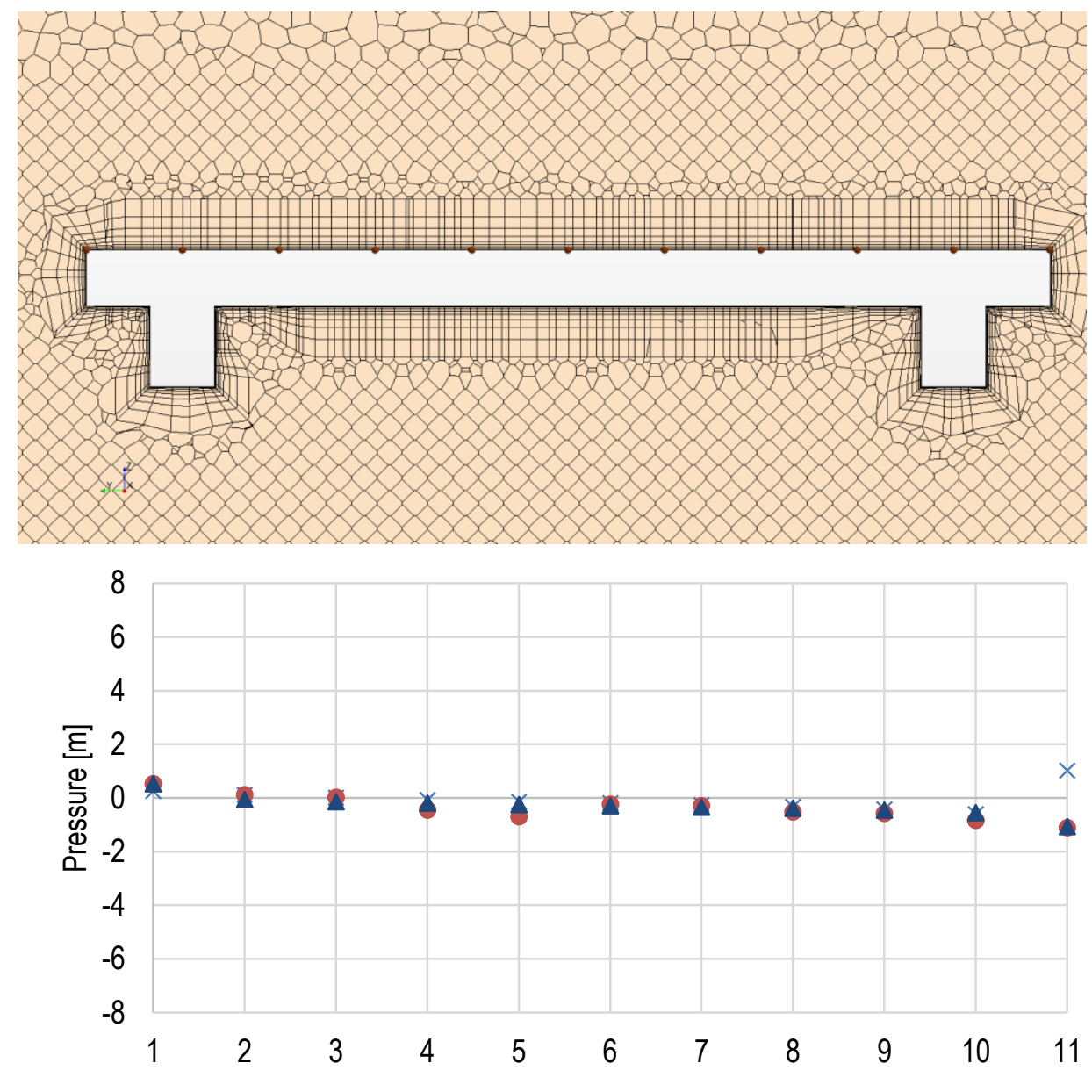

$\times$ k-epsilon, polyhedral mesh, $Y_{+}=1.1 \quad$ LES, polyhedral mesh, $Y_{+}=1.1$

$\Delta$ k-epsilon, polyhedral mesh, $Y+=20.6$

Figure 4-4: Pressure on the symmetry line of the top surface of downstream deck, (a) location of measured points, (b) pressure values 
(a)

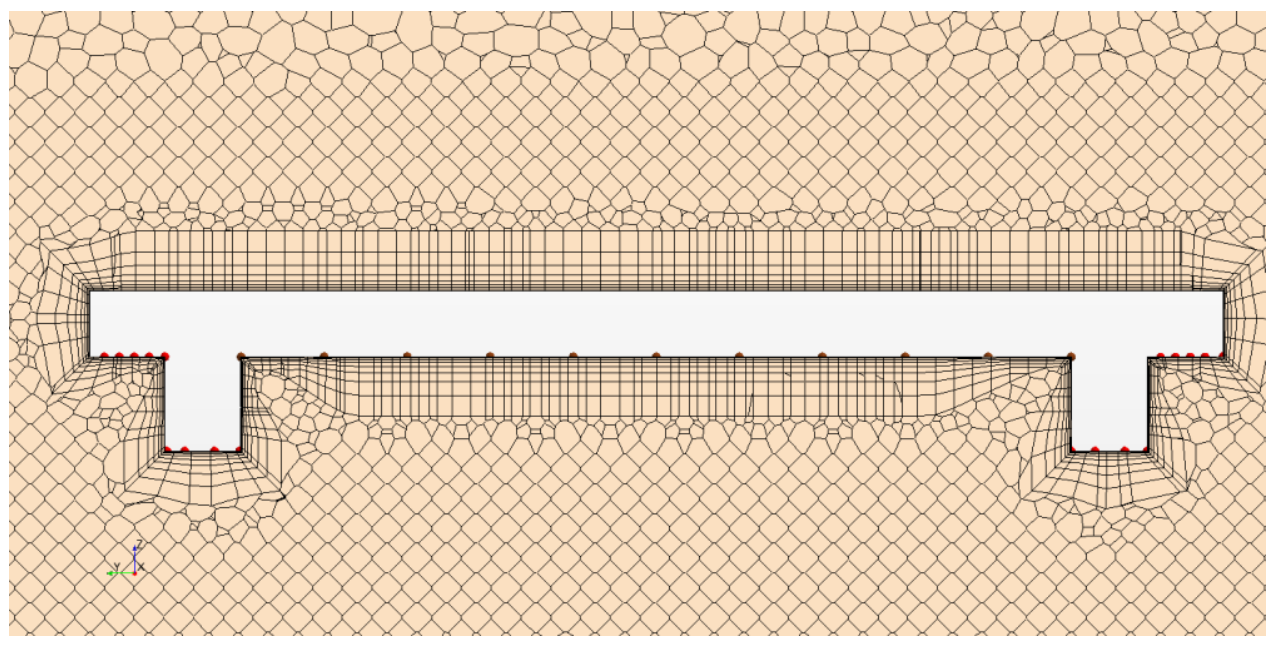

(b)

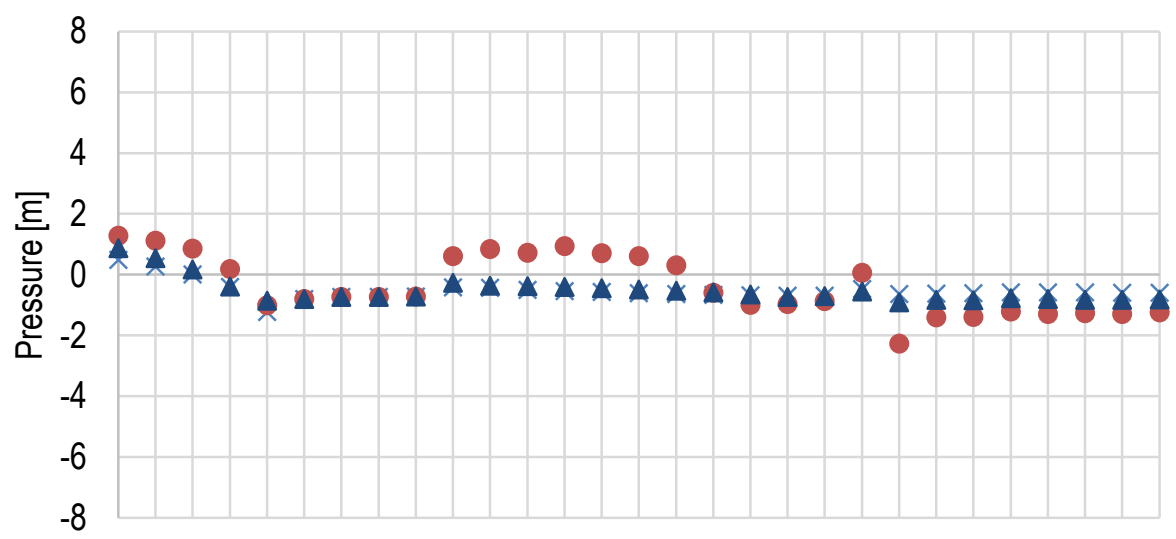

1234567891011121314151617181920212223242526272829

$\times$ k-epsilon, polyhedral mesh, $Y+=1.1 \quad$ LES, polyhedral mesh, $Y+=1.1$

$\Delta$ k-epsilon, polyhedral mesh, $Y+=20.6$

Figure 4-5: Pressure on the symmetry line of the bottom surface of downstream deck, (a) location of measured points, (b) pressure values 
(a)

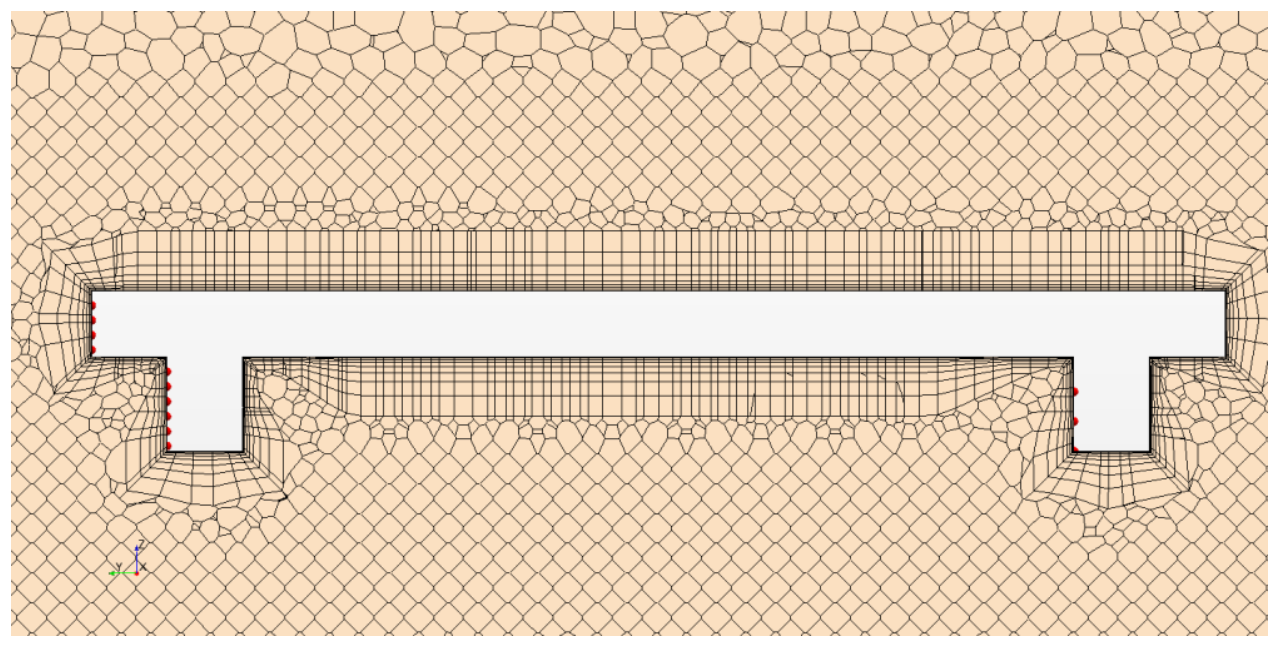

(b)

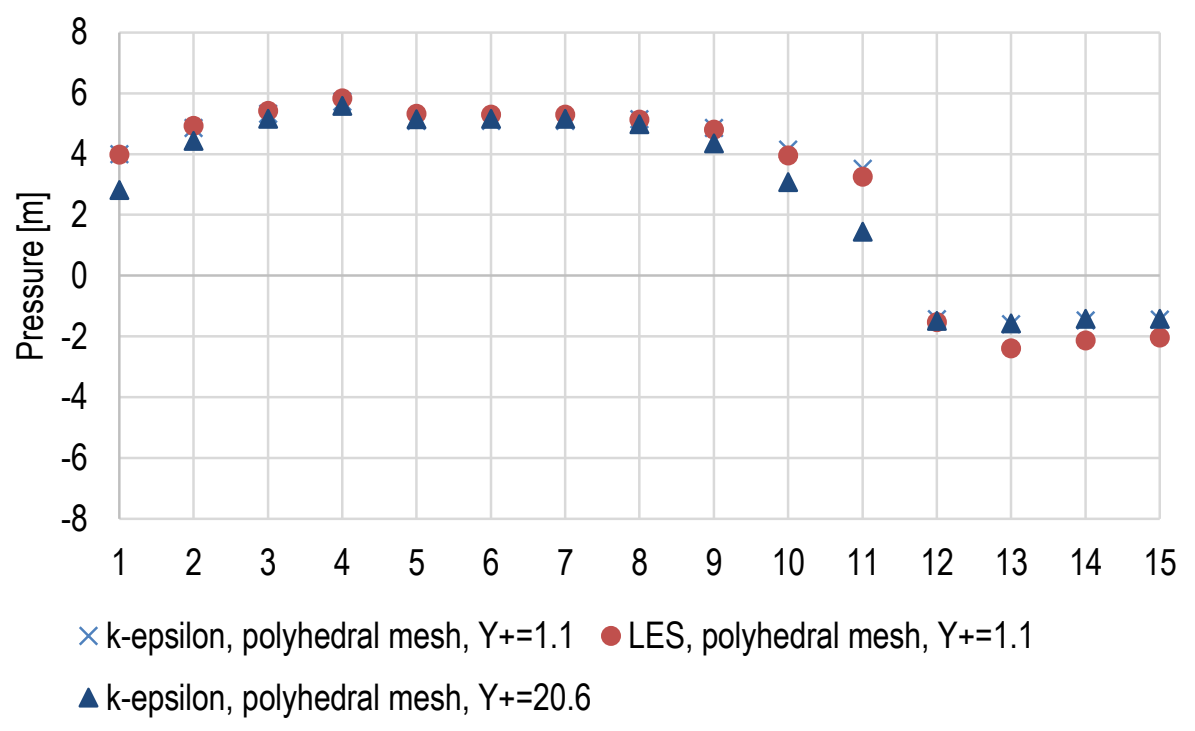

Figure 4-6: Pressure on the symmetry line of the upstream surface of the upstream deck, (a) location of measured points, (b) pressure values 
(a)

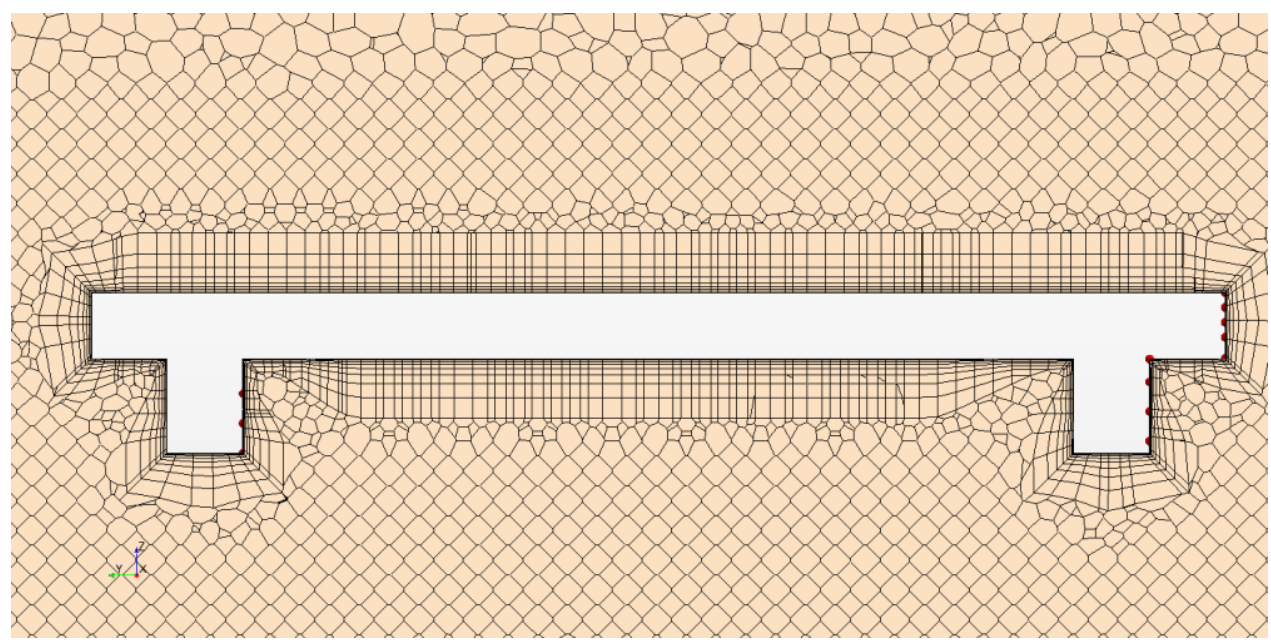

(b)

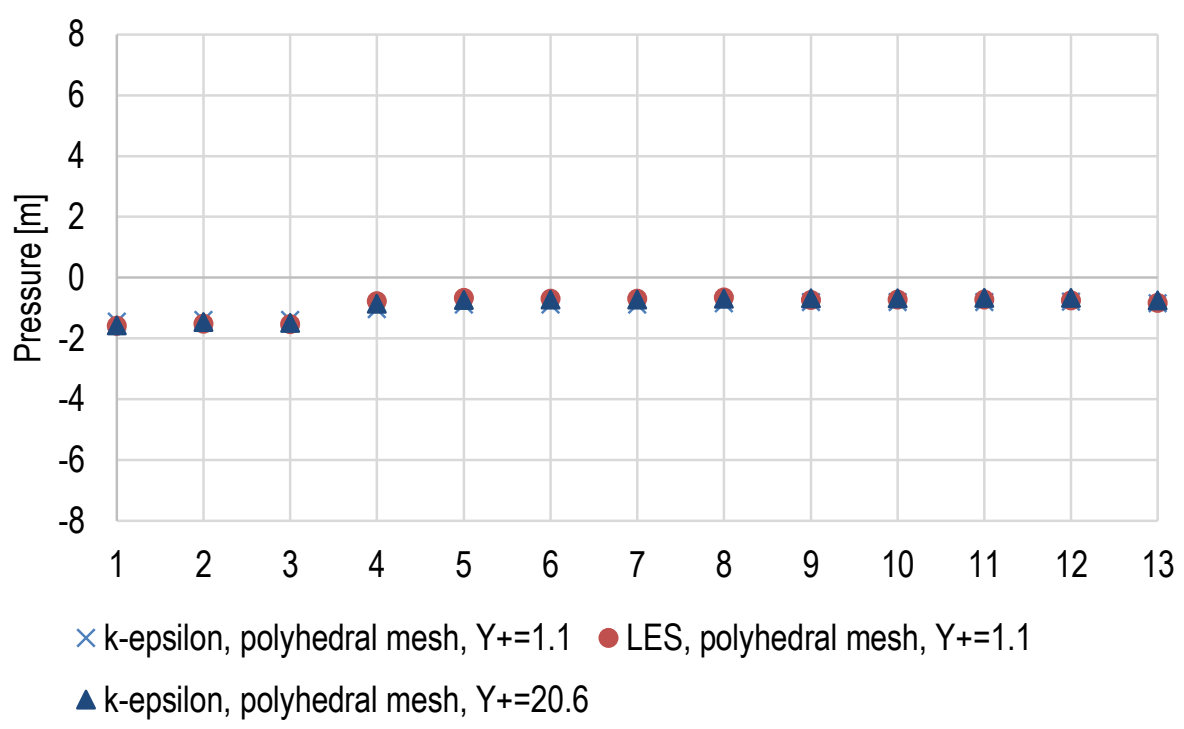

Figure 4-7: Pressure on the symmetry line of the downstream surface of upstream deck, (a) location of measured points, (b) pressure values 
(a)

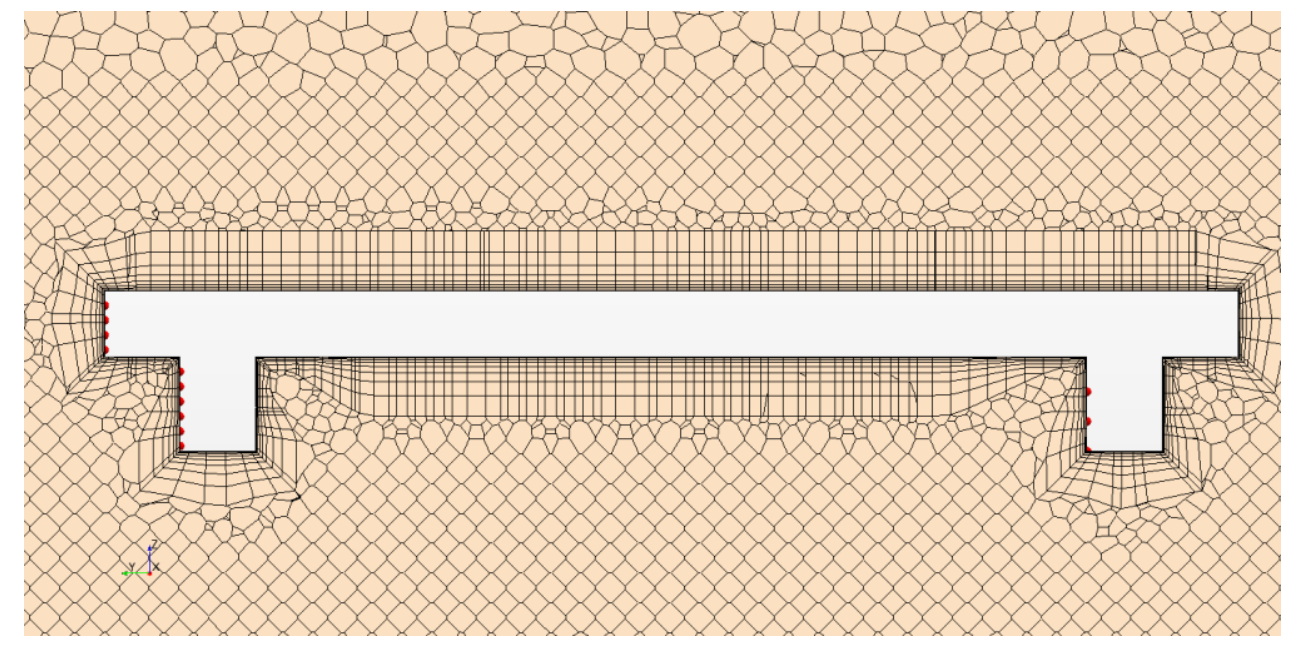

(b)

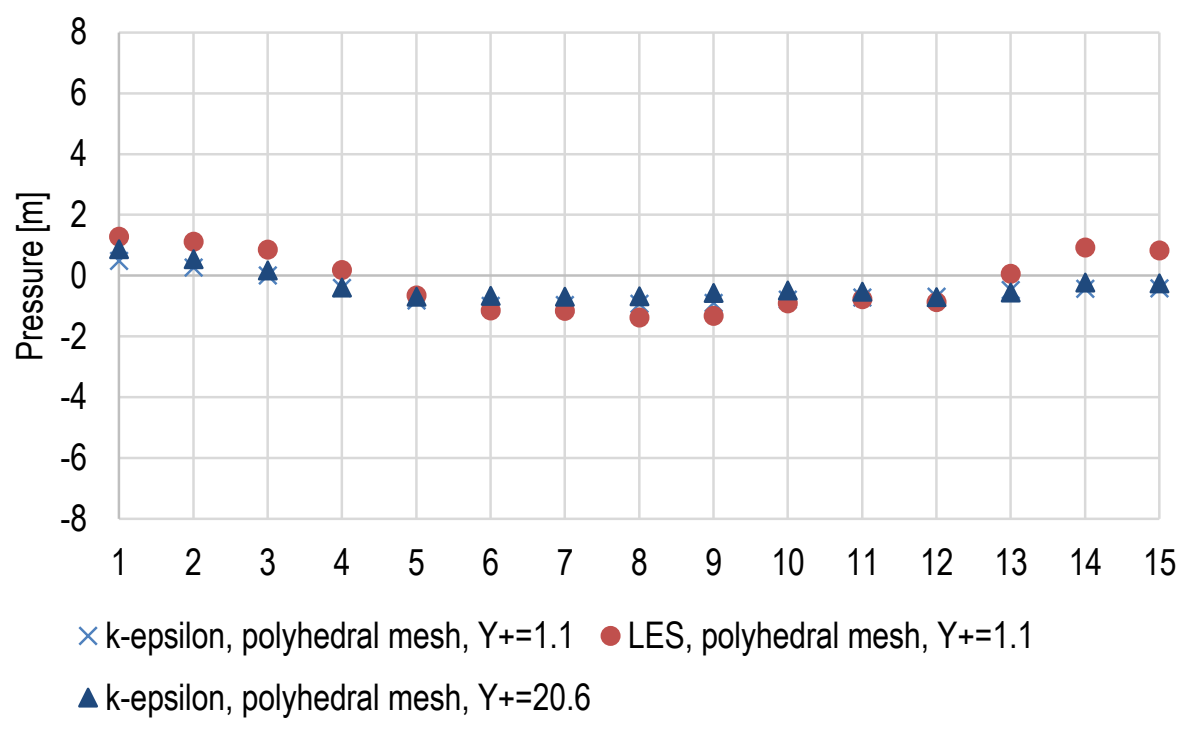

Figure 4-8: Pressure on the symmetry line of the upstream surface of the downstream deck , (a) location of measured points, (b) pressure values 
(a)

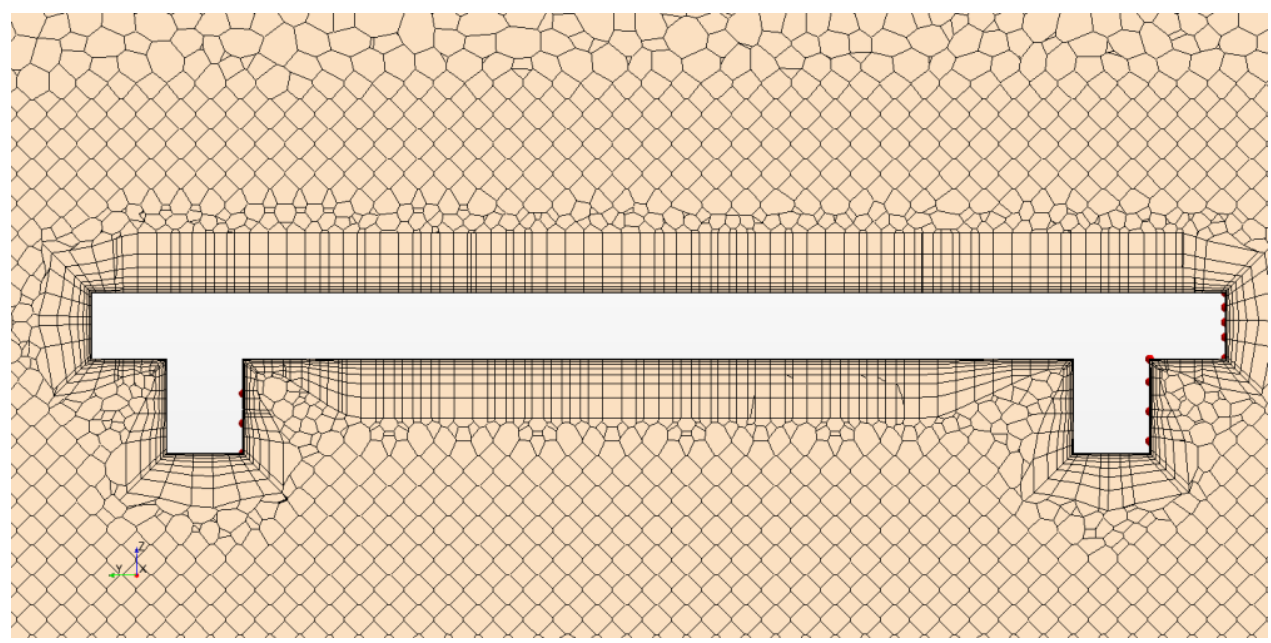

(b)

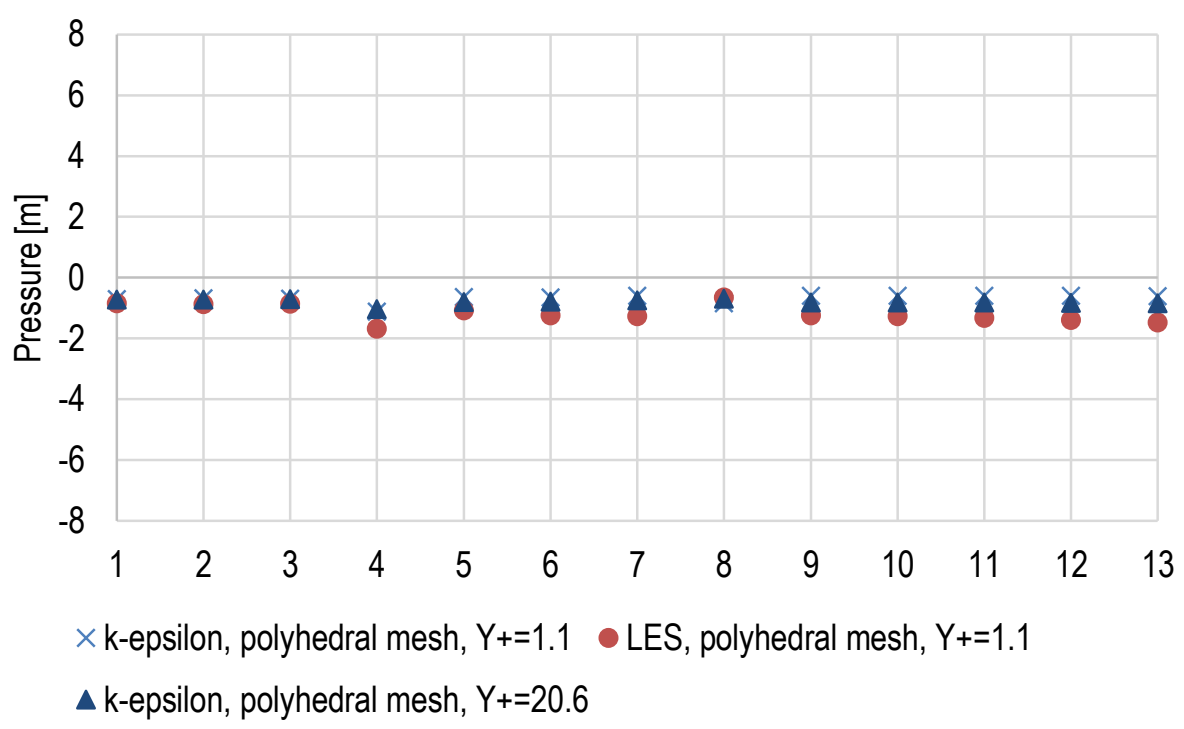

Figure 4-9: Pressure on the symmetry line of the downstream surface of the downstream deck, (a) location of measured points, (b) pressure values 


\subsection{Influence of gap-to-width ratio}

The influence of the horizontal distance between two decks on pressure and velocity fields around them is investigated in this section. The gap-to-width ratios in the model cases are: $0.13,0.26$, 0.52, and 0.79. Table 4-1 summarizes the maximum and the minimum pressure and velocity values acting on the upstream deck with different spacing between decks. The differences in values are very small, less than $4 \%$. Table 4-2 shows field plots of pressure and velocity at various deck spacings.

Table 4-1: Extreme values of pressure and velocity for a twin deck with varying gap

\begin{tabular}{|c|c|c|c|}
\hline & \multicolumn{2}{|c|}{ Pressure [Pa] } & Velocity [m/s] \\
\hline L/B & min & $\max$ & $\max$ \\
\hline 0.13 & -8.37 & 5.38 & 3.81 \\
\hline 0.26 & -8.38 & 5.40 & 3.80 \\
\hline 0.52 & -8.36 & 5.4 & 3.8 \\
\hline 0.79 & -8.39 & 5.40 & 3.81 \\
\hline
\end{tabular}

Table 4-2: Pressure and velocity fields around twin deck with varying gap

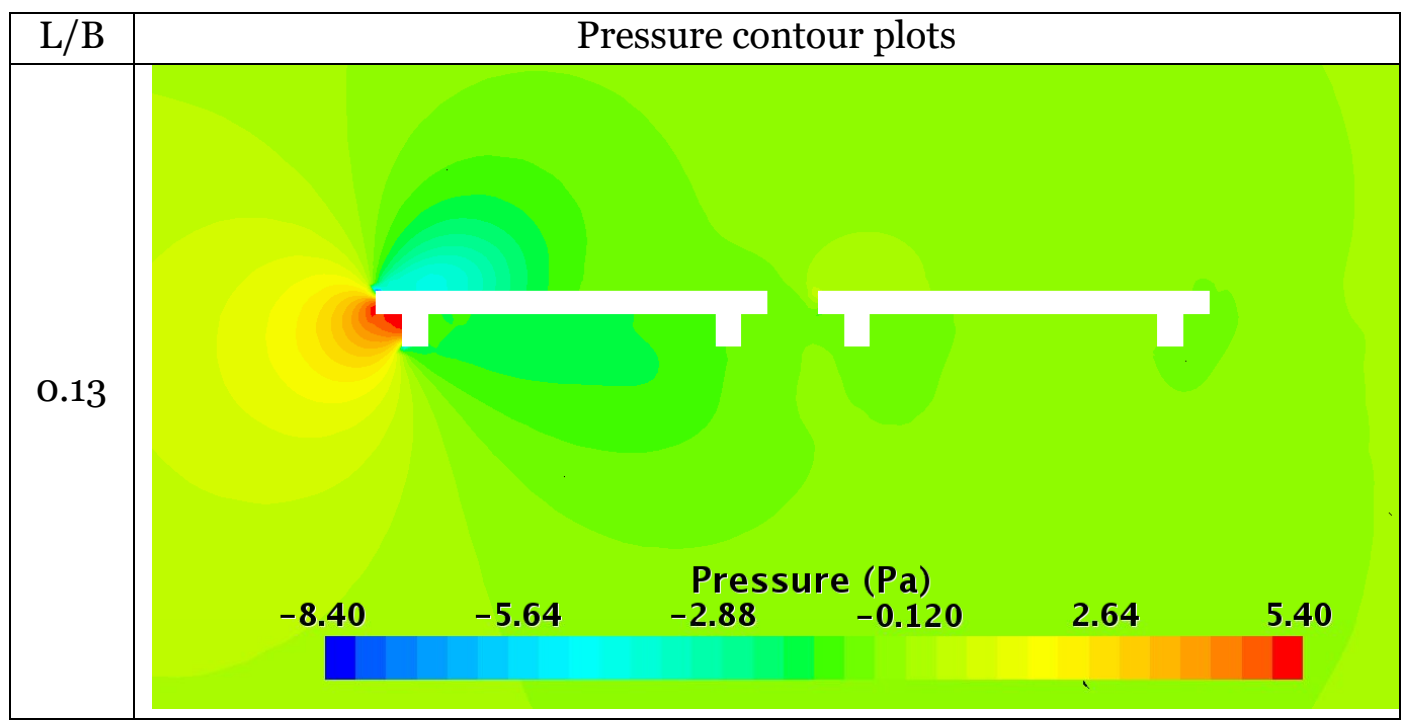




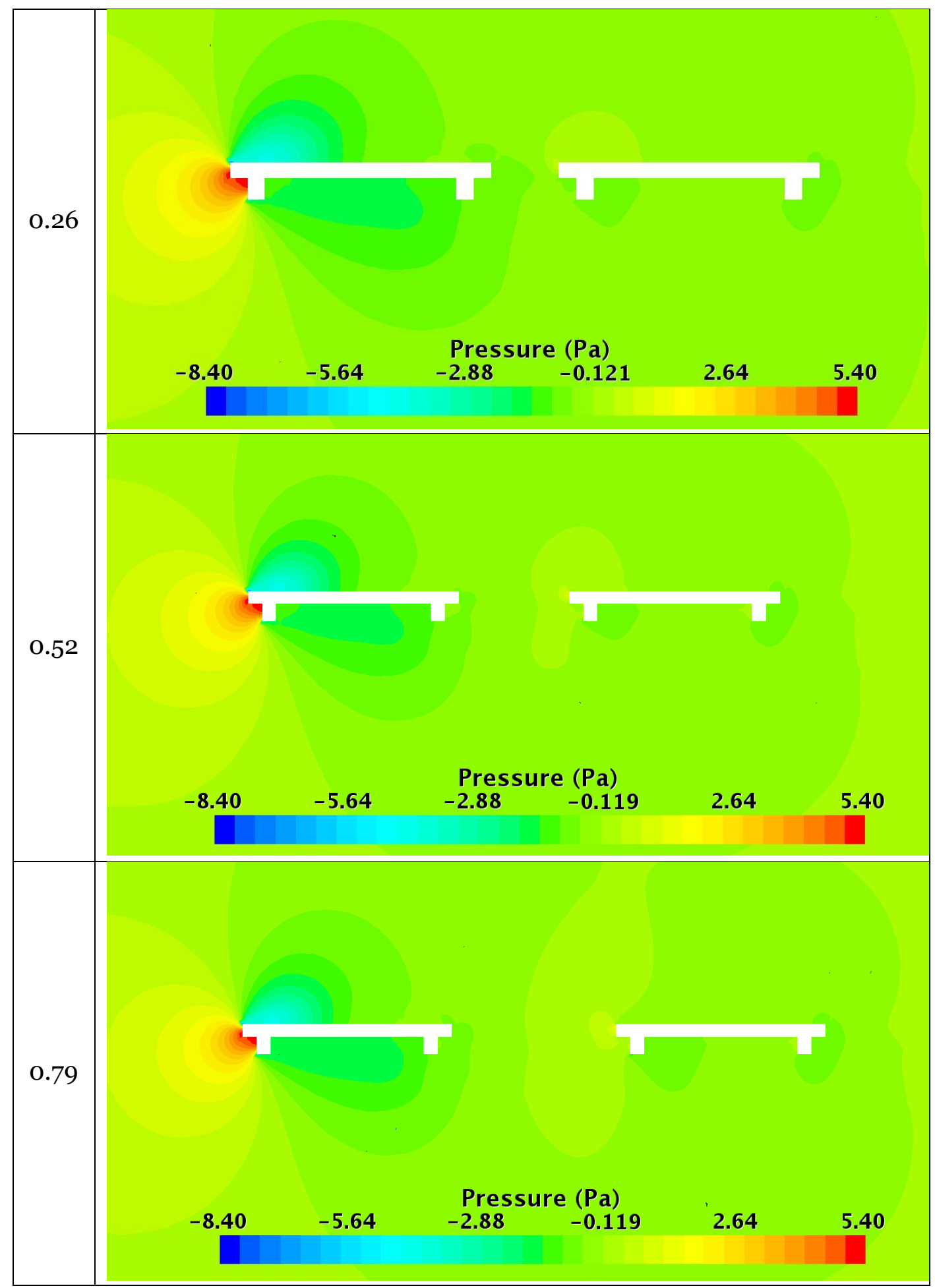

Investigation of Aerodynamic Interference between Twin Deck Bridges

Page $\mid 28$ 
Table 4-3: Velocity field contour plots for a twin deck bridge at varying gap

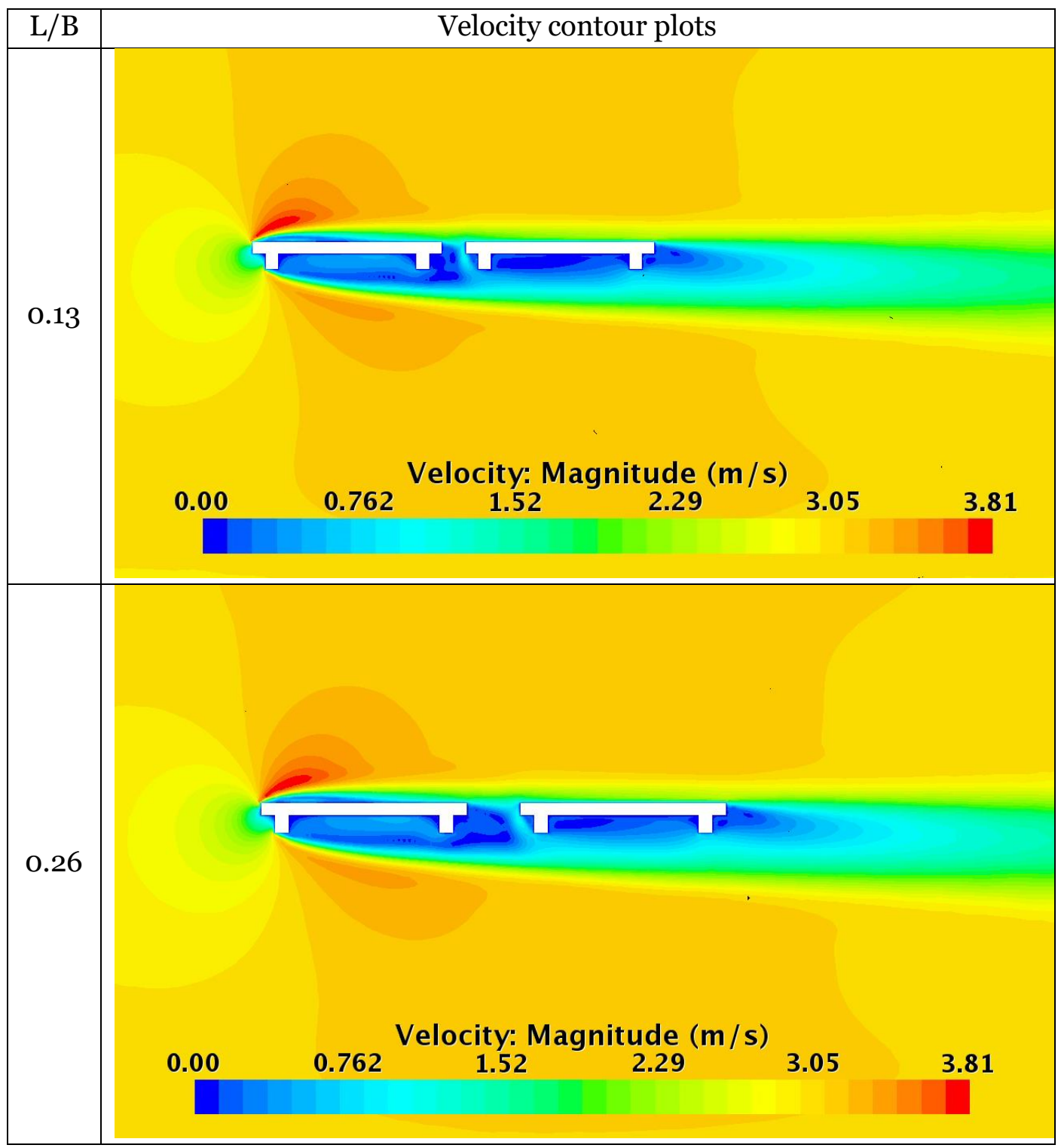




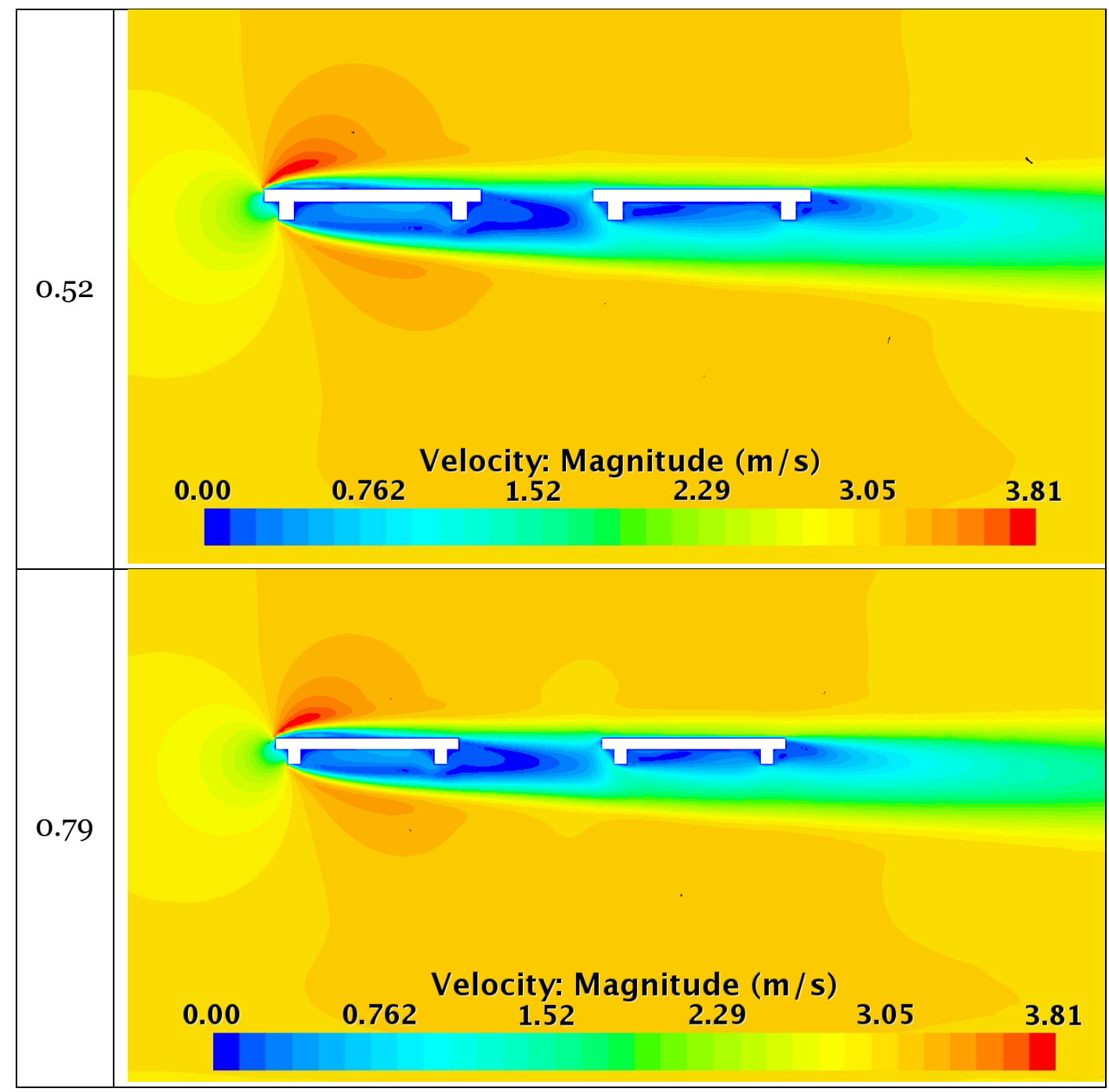

The combined results for drag, lift, and pitch moment acting on a single deck and a twin deck are shown in Figure 4-10, Figure 4-11, and Figure 4-12. A comparison of force components on deck 1 (the upstream deck in the twin deck bridge system) and the single deck model show that there is only a slight difference in values. The deck spacing does not have a big influence on the drag forces acting on deck 1, which is almost constant and is equal to $0.41 \mathrm{~N}$. The drag is lower on the downstream deck, because it is located in the upstream deck wake. This effect diminishes as the gap increases. A change of spacing from $0.05 \mathrm{~m}$ to $0.3 \mathrm{~m}(\mathrm{~L} / \mathrm{B}=0.13$ and 0.79$)$ causes the drag force on deck 2 (downstream deck in the twin deck bridge system) to increase from $0.15 \mathrm{~N}$ to 0.25 $\mathrm{N}$, and the pitch moment changes from -0.016 Nm to -0.032 Nm. In contrast, lift force does not vary significantly. The values of lift force for the upstream deck are 15-20\% higher than for a single deck and they are almost constant (varying between $0.45 \mathrm{~N}$ and $0.48 \mathrm{~N}$ ) regardless the gap. The lift force on the downstream deck increases as the gap gets bigger, from $0.11 \mathrm{~N}$ for the smallest gap to $0.135 \mathrm{~N}$ for $0.2 \mathrm{~m}$ gap $(\mathrm{L} / \mathrm{B}=0.52)$. 


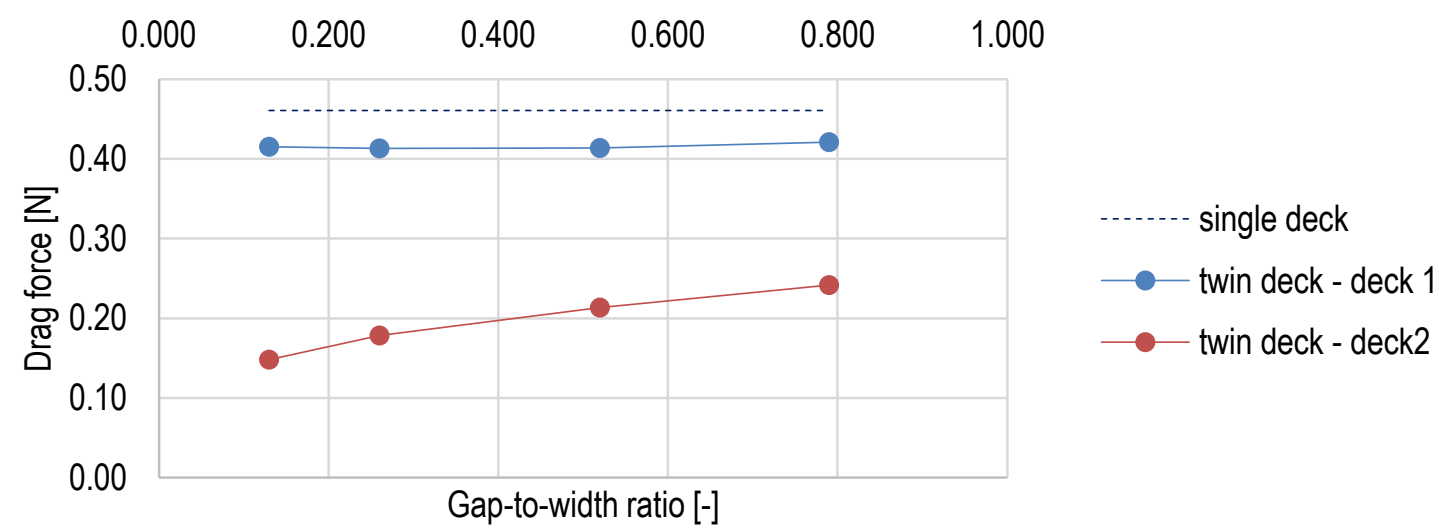

Figure 4-10: Gap-to width ratio influence on the drag force in parallel flow. Deck 1 - upstream deck, deck 2 - downstream deck.

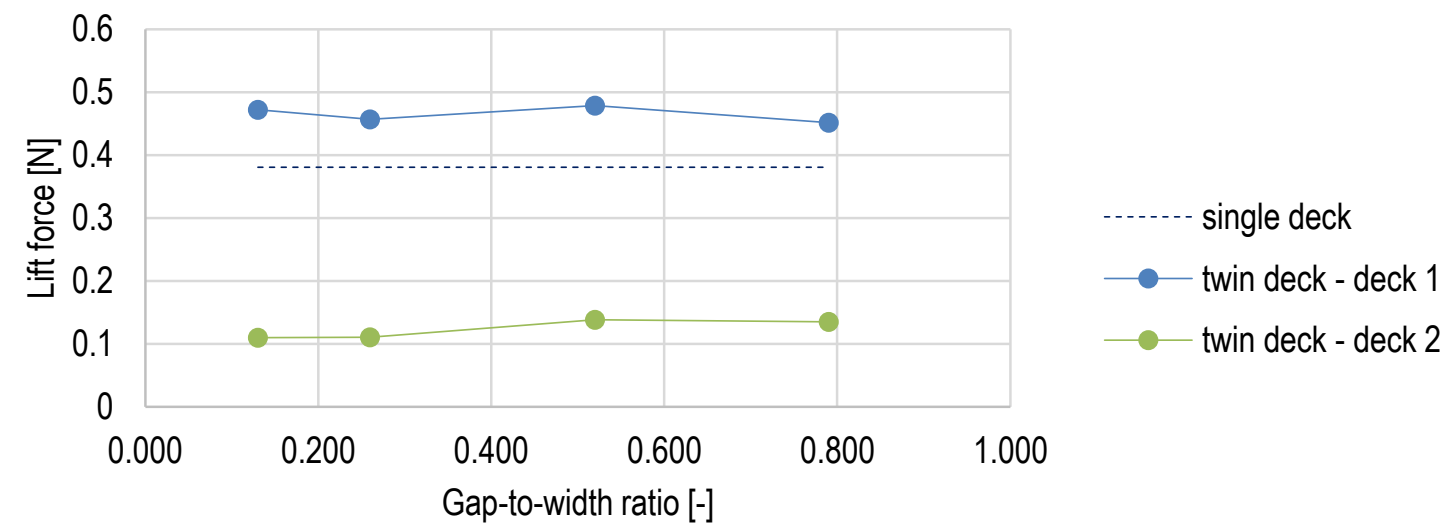

Figure 4-11: Gap-to width ratio influence on the lift force in parallel flow. Deck 1 - upstream deck, deck 2 - downstream deck.

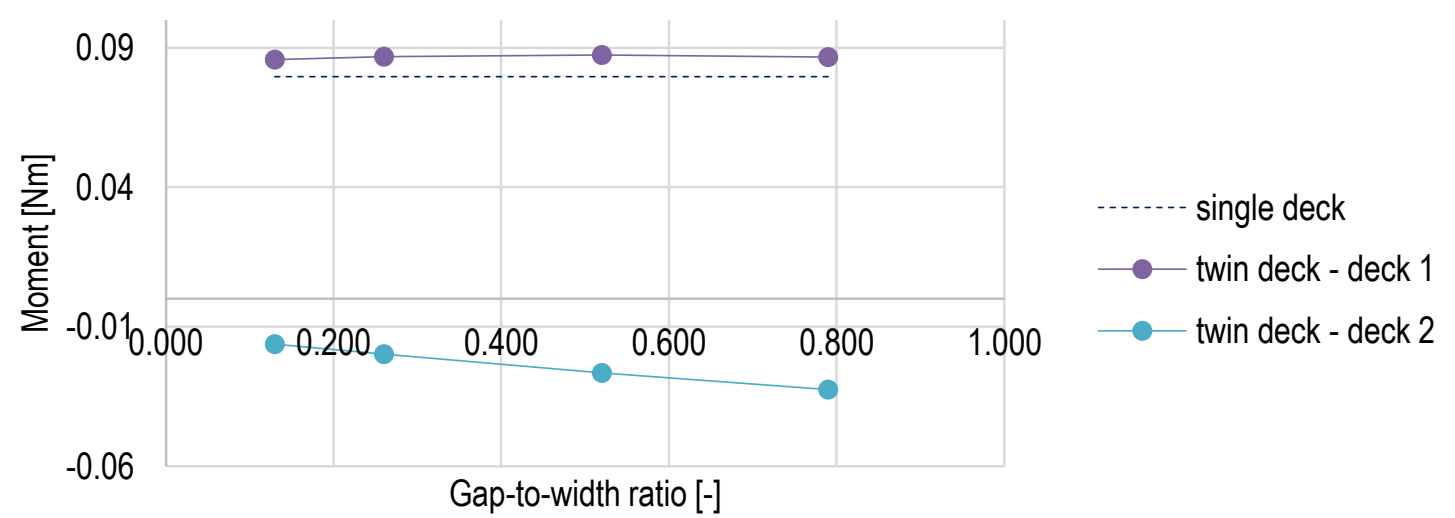

Figure 4-12: Gap-to width ratio influence on the pitch moment in parallel flow. Deck 1 upstream deck, deck 2 - downstream deck. 


\subsection{Influence of varying angle of attack}

A set of five angles of attack were tested, which ranged from -10 degrees to 10 degrees in 5 degree increments. Pressure contour plots for the twin deck model with a $0.1 \mathrm{~m}$ gap $(\mathrm{L} / \mathrm{B}=0.26)$ are collected in Table 4-4 and velocity contour plots for the same setup are presented in Table 4-5.

Table 4-4: Pressure field contour plots for a twin deck bridge with $0.1 \mathrm{~m}$ gap $(\mathrm{L} / \mathrm{B}=0.26)$ at varying angle of attack

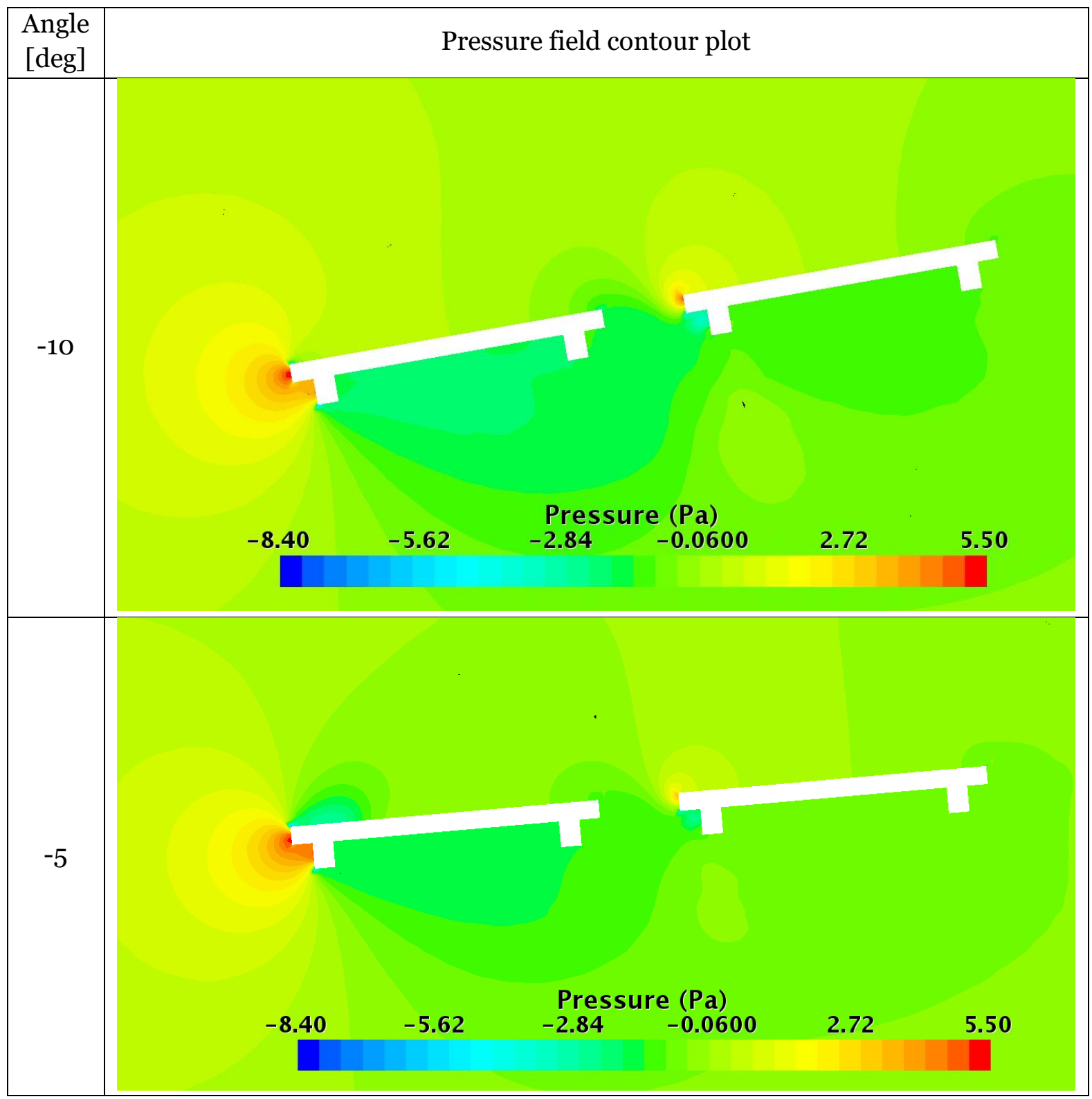




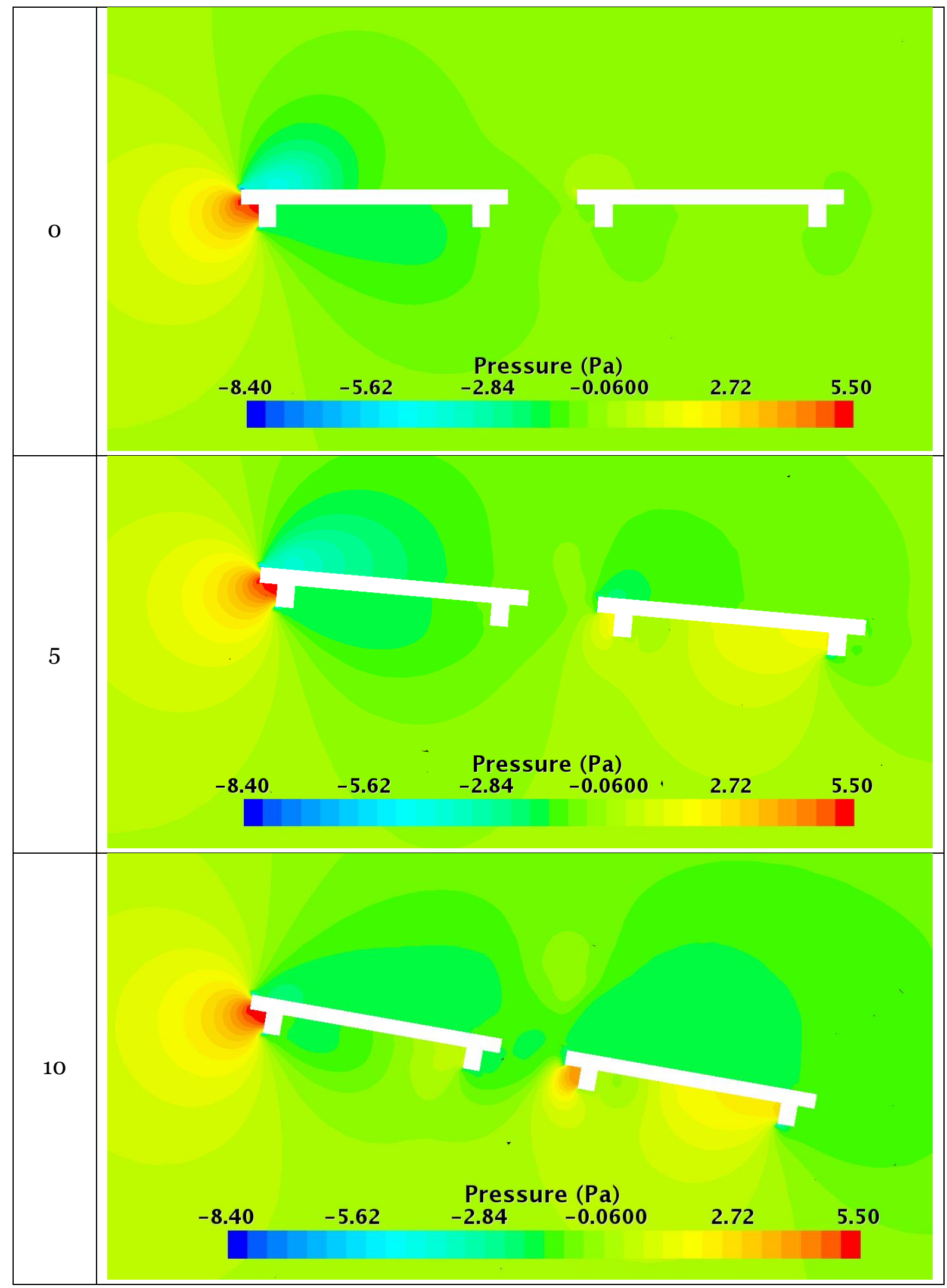

Investigation of Aerodynamic Interference between Twin Deck Bridges

Page $\mid 33$ 
Table 4-5: Velocity field contour plots for a twin deck bridge with $0.1 \mathrm{~m}$ gap $(\mathrm{L} / \mathrm{B}=0.26)$ at varying angle of attack

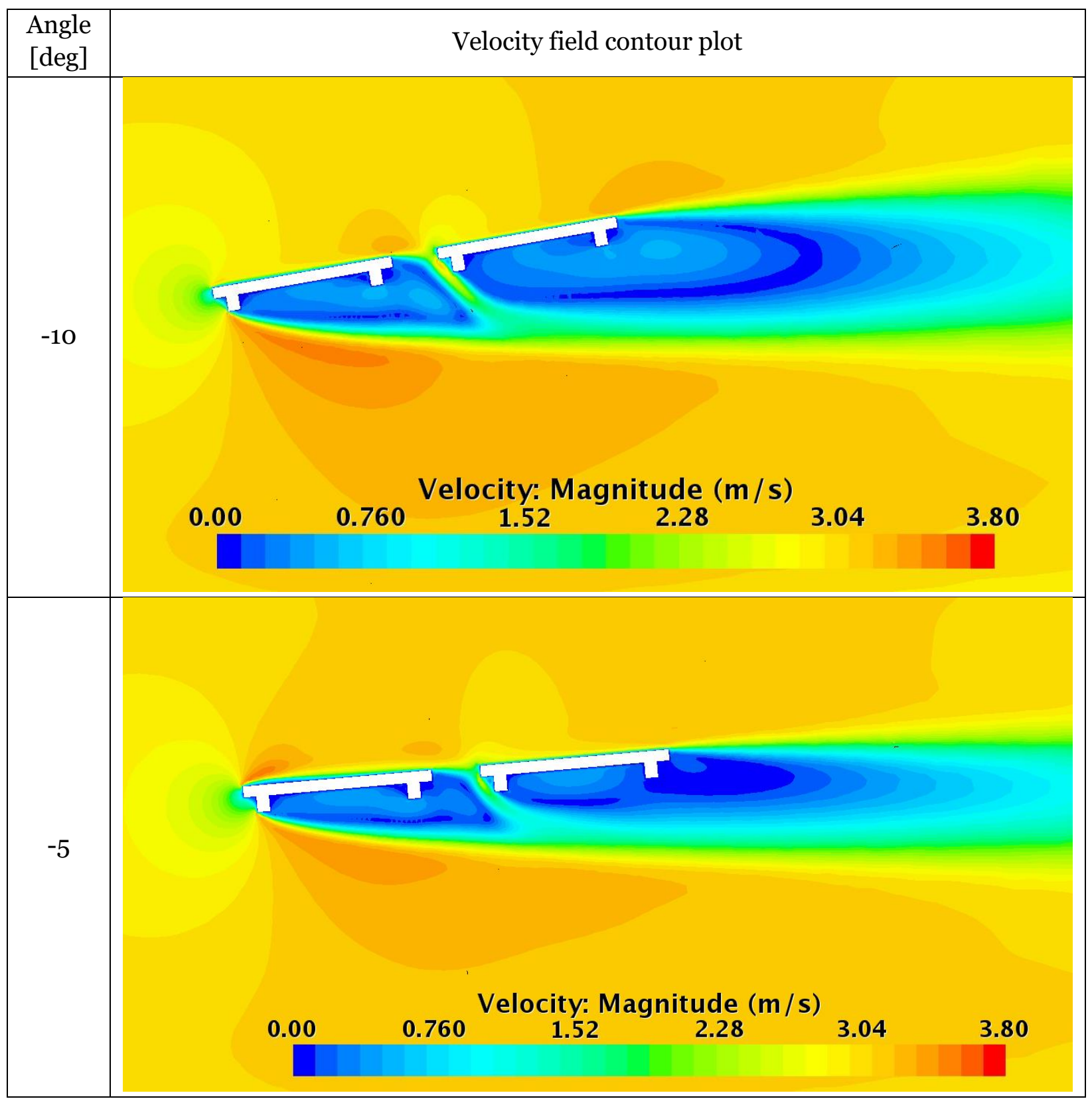




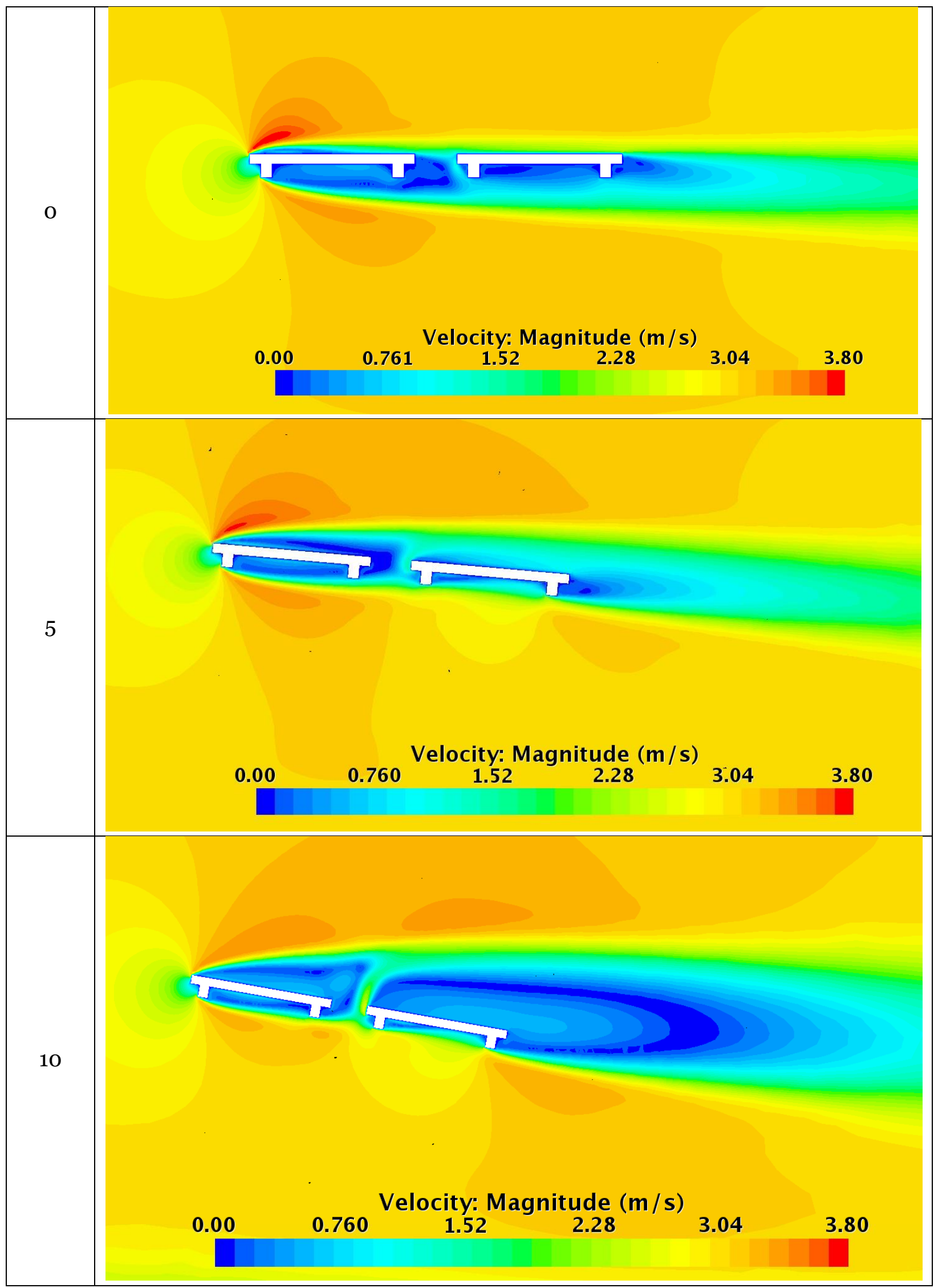

Investigation of Aerodynamic Interference between Twin Deck Bridges

Page $\mid 35$ 
Maximum and minimum values of pressure acting on deck surfaces, with respect to changing flow angle of attack, are presented in Table 4-6. Differences in maximum values are not significant with the maximum equal $5.52 \mathrm{~Pa}$ at $-5 \mathrm{deg}$ angle. The minimum value for a twin deck at parallel flow is close to the single deck case and it increases with the increase of the absolute value of angle of attack. The maximum velocity in the vicinity of the decks does not experience big changes and reaches a maximum of $3.8 \mathrm{~m} / \mathrm{s}$ in parallel flow.

Table 4-6: Extreme values of pressure and velocity for a twin deck

\begin{tabular}{|c|c|c|c|}
\hline Angle [deg] & \multicolumn{2}{|c|}{ Pressure [Pa] } & Velocity [m/s] \\
\hline & $\min$ & $\mathbf{m a x}$ & $\mathbf{m a x}$ \\
\hline-10 & -6.31 & 5.48 & 3.50 \\
\hline-5 & -7.11 & 5.52 & 3.53 \\
\hline 0 & -8.38 & 5.40 & 3.80 \\
\hline 5 & -7.45 & 5.37 & 3.74 \\
\hline 10 & -5.21 & 5.46 & 3.43 \\
\hline
\end{tabular}

The variation of the force and angle of attack is illustrated in Figure 4-13 to Figure 4-15 for a twin deck with a $0.1 \mathrm{~m}$ distance between decks (gap ratio 0.26). Figure 4-13 shows plots of the drag forces acting on the decks. The force stays positive and increases with the increase of the absolute value of the angle, and reaches the maximum of approximately $0.6 \mathrm{~N}$ at 10 degrees. Drag on the upstream deck has values that are close to the single deck model for the selected flow directions. For upstream/downstream bridge deck, the biggest difference can be seen for -10 deg angle, where the force equals $0.35 \mathrm{~N}$ (as compared to $0.6 \mathrm{~N}$ ), whereas for 10 deg the values are the closest to each other (and equal approximately o.6 N). The lift force, displayed in Figure 4-14, changes sign with the angle of attack. This tendency is true for the single deck model as well as for the twin deck model. For negative values of the angle of attack the lift force assumes negative values (the lowest: $-0.61 \mathrm{~N}$ for the downstream deck in a twin deck and -0.736 N for upstream deck in a twin deck), goes through zero for angles in the range from -5 deg to o deg, and reaches positive values for non-negative angles. The highest value of the lift force was obtained for the downstream deck in the twin deck bridge at $10 \mathrm{deg}$ angle. It is almost two times higher for the downstream deck in the twin deck bridge than the upstream deck (it is equal to $1.5 \mathrm{~N}$ and $0.84 \mathrm{~N}$ respectively). The lift force acting on the single deck at 10 deg angle falls in between these values and is equal $1.1 \mathrm{~N}$. The pitch moment at various flow directions is shown in Figure 4-15. The pitch moment for the upstream deck in a twin deck bridge reaches a maximum of $0.09 \mathrm{Nm}$ for parallel flow and decreases as the absolute value of the angle of attack increases. The values of the pitch moment for the downstream deck in a twin deck are negative for most of the angles of attack and equal between -0.02 $\mathrm{Nm}$ to $-0.03 \mathrm{Nm}$. At the angle of attack equal to $5 \mathrm{deg}$, the pitch moment is smaller and equals $0.005 \mathrm{Nm}$. 


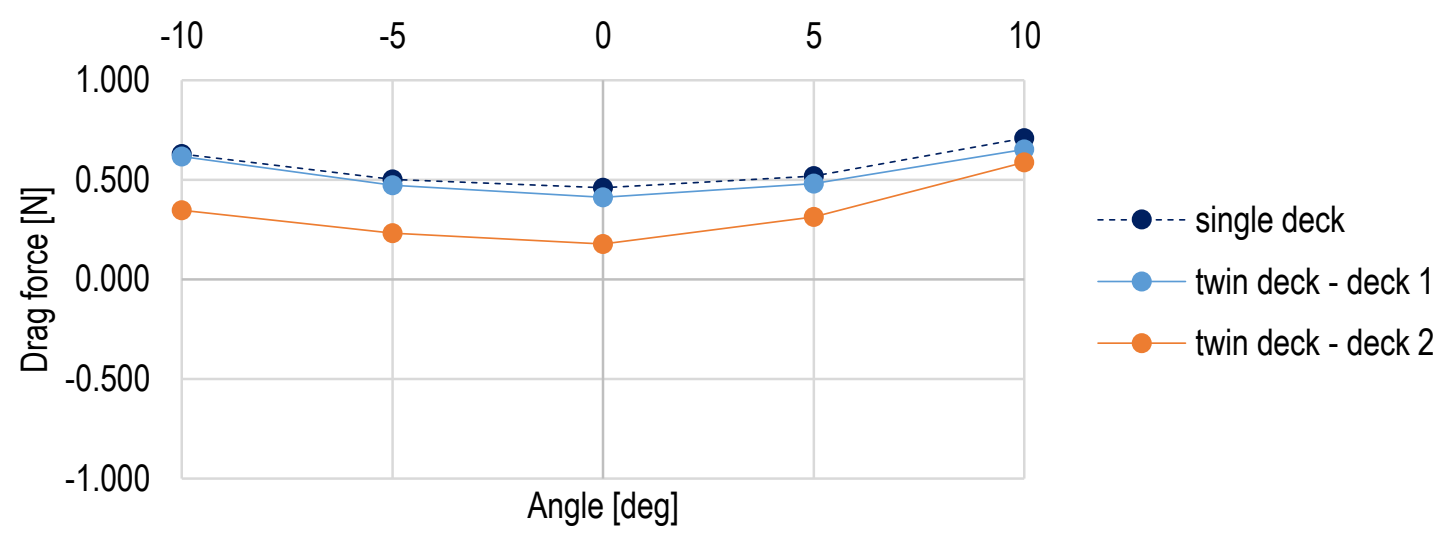

Figure 4-13: Influence of angle of attack on drag force, L/B=0.13. Deck 1 - upstream deck, deck 2 - downstream deck.

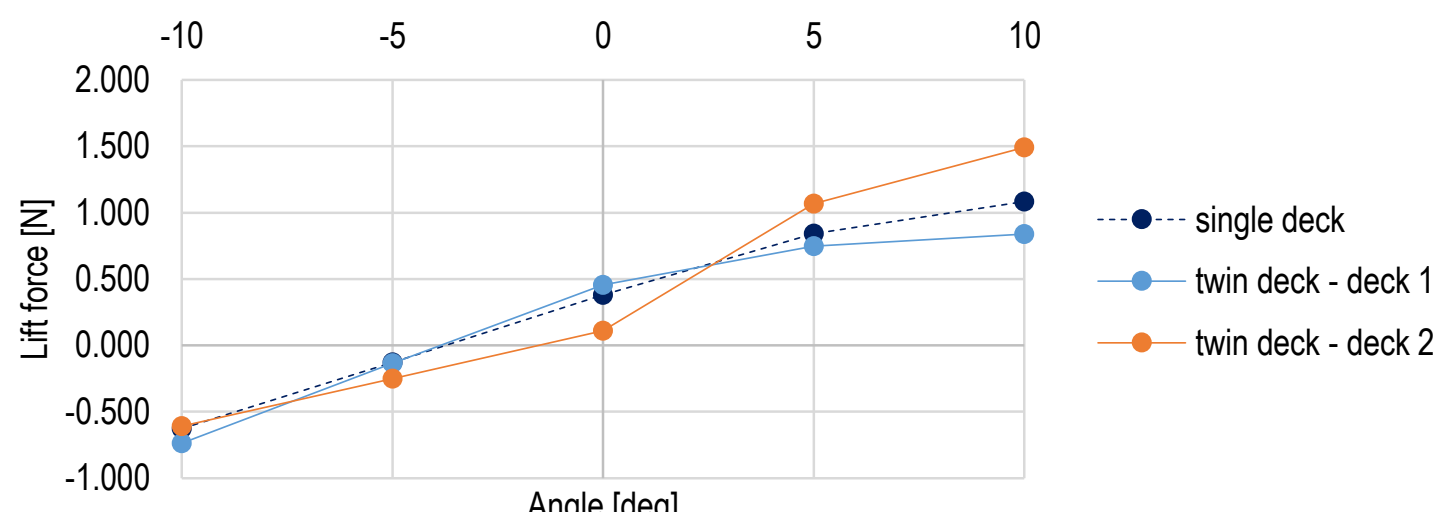

Figure 4-14: Influence of angle of attack on lift force, L/B=0.13. Deck 1 - upstream deck, deck 2 - downstream deck.

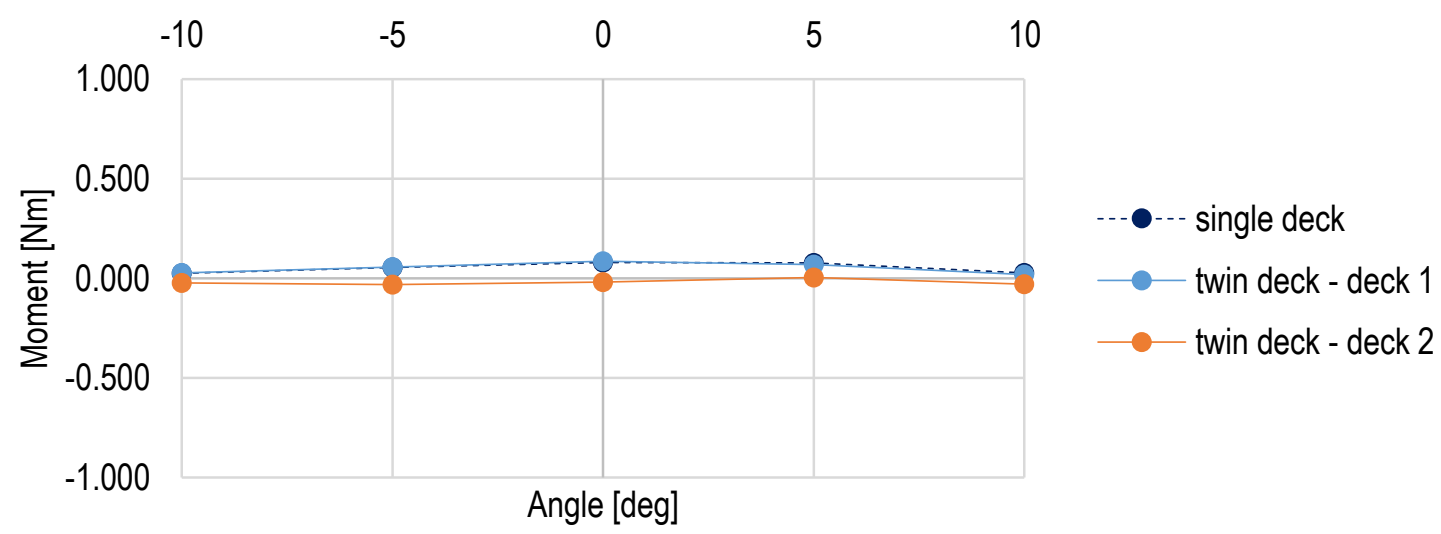

Figure 4-15: Influence of angle of attack on pitch moment, L/B=0.13. Deck 1 - upstream deck, deck 2 - downstream deck. 


\section{Dynamic response}

The aerodynamic response of the decks can be investigated with the use of the Dynamic Fluid Body Interaction (DFBI) solver available in STAR-CCM+. The solver accounts for six degrees of freedom of body motions, uses mesh morphing to maintain a high quality mesh when bodies move in the domain, and handles solving for fluid transport through the deforming mesh. The URANS solver with the k- $\varepsilon$ turbulence model is used to solve for the fluid flow.

The following model configurations are taken into account and compared:

- decks are stationary

- DFBI configuration 1: both decks are suspended on springs,

- DFBI configuration 2: deck 1 is suspended on springs and deck 2 is constrained,

- DFBI configuration 3: deck 1 is constrained and deck 2 is suspended on springs.

The decks are modeled as rigid bodies and each of them is suspended on 8 springs of assumed length $(0.5 \mathrm{~m})$ and stiffness $(1000 \mathrm{~N} / \mathrm{m})$. Mass, center of mass, and all components of moment of inertia of the deck model have to be provided. These values are obtained using the geometry property computation capabilities of the LS-DYNA, LS-PrePost preprocessor [27] with an assumed material density of $1300 \mathrm{~kg} / \mathrm{m}^{3}$. The total mass of the deck equals $20 \mathrm{~kg}$. The center of mass is located at $\mathrm{Z}=-0.015 \mathrm{~m}$, with $\mathrm{Z}=\mathrm{O} \mathrm{m}$ on the top surface of the deck. The moments of inertia are: $I_{x x}=11.6 \mathrm{~kg} \mathrm{~m}^{2}, I_{y y}=0.69 \mathrm{~kg} \mathrm{~m}^{2}$, and $I_{z z}=12.3 \mathrm{~kg} \mathrm{~m}^{2}$, about x, y, and $\mathrm{z}$ axis respectively.

The release time is specified to be $30 \mathrm{sec}$. The period before the release time allows an initial flow solution to be computed including fluid forces on the deck. After steady state flow on stationary decks is achieved, the body suspended on springs is released. The body forces, including fluid forces, can now move the deck. The initial time and the ramp up time (together with the damping forces) should be long enough to reduce sudden non-physical application of the gravitational force to a reasonable level. This time depends on several parameters, like mass of the deck, stiffness of the springs, size of the domain, and cell sizes. It should be selected for each simulation. If the full gravitational force is applied at once, the mesh deformation can be big enough to cause zerovolume cells to appear and the run ends with an error. A ramp up of the force avoids this problem.

The deck suspended on springs is allowed to move only vertically and rotationally along the centerline of the deck. All other rigid body motions are constrained. The stationary deck is positioned $2.46 \mathrm{~cm}$ below the initial location of the moveable deck to take into account the elongation of top springs due to the weight of the deck. When the body is released, i.e. when the springs are activated in the model, it still moves downwards, due to gravity. The displacement oscillates around a constant value with a decreasing amplitude. No structural damping is involved in the simulation; therefore, this change is a result of aerodynamic damping.

Force components were recorded and compared for both decks. The forces acting on stationary decks are established during the first 30 sec of the simulation time. The drag force acting on the upstream deck converges to a value of $0.413 \mathrm{~N}$ and the force acting on the downstream deck converges to $0.178 \mathrm{~N}$. The lift forces for the upstream and downstream decks are $0.455 \mathrm{~N}$ and $0.108 \mathrm{~N}$, accordingly, and pitch moments are equal to $0.086 \mathrm{Nm}$ and -0.02 Nm for the upstream and downstream decks, respectively. 


\subsection{Configuration 1: both decks suspended on springs}

Figure 5-1 illustrates DFBI configuration 1, where both decks are suspended on elastic springs. Their vertical motion and rotation are illustrated in Figure 5-2 for the time after the springs are activated. High initial amplitudes, reaching $4.7 \mathrm{~cm}$, decrease in time due to aerodynamic damping. After $100 \mathrm{sec}$ of simulation time they are equal $1.1 \mathrm{~mm}$. Both decks oscillate around the same value of $-0.0246 \mathrm{~m}$, which is a result of the elongation of the upper springs and compression of the lower springs due to the weight of the model. The amplitude of the motion for the downstream deck is higher than for the upstream deck. A Fast Fourier Transform (FFT) was used to establish the vibration frequencies of both decks. The calculations show that the first frequency of the decks is the same and equals $3.14 \mathrm{~Hz}$, as shown in Figure 5-3.

Rotations of the decks around local center lines are presented in Figure 5-4. Slightly higher amplitude of the rotational angle is reported for the downstream deck, which is 1 degree compared to 0.7 degrees for the upstream deck. Rotational frequency is equal to $3.24 \mathrm{~Hz}$, as shown in Figure 5-5. Plots of forces acting on the decks are shown in Figure 5-6. Table 5-1 combines the force values for the stationary twin deck and a twin deck suspended on elastic springs.

The velocity field is recorded and examined in more detail after the activation of the springs. Table 5-2 shows how the velocity changes around the decks in consecutive time steps. The velocity vector field is plotted on the middle plane. Table 5-3 illustrates velocity streamlines changing in time. The seeds for the streamlines lay on the domain inlet at the same height.

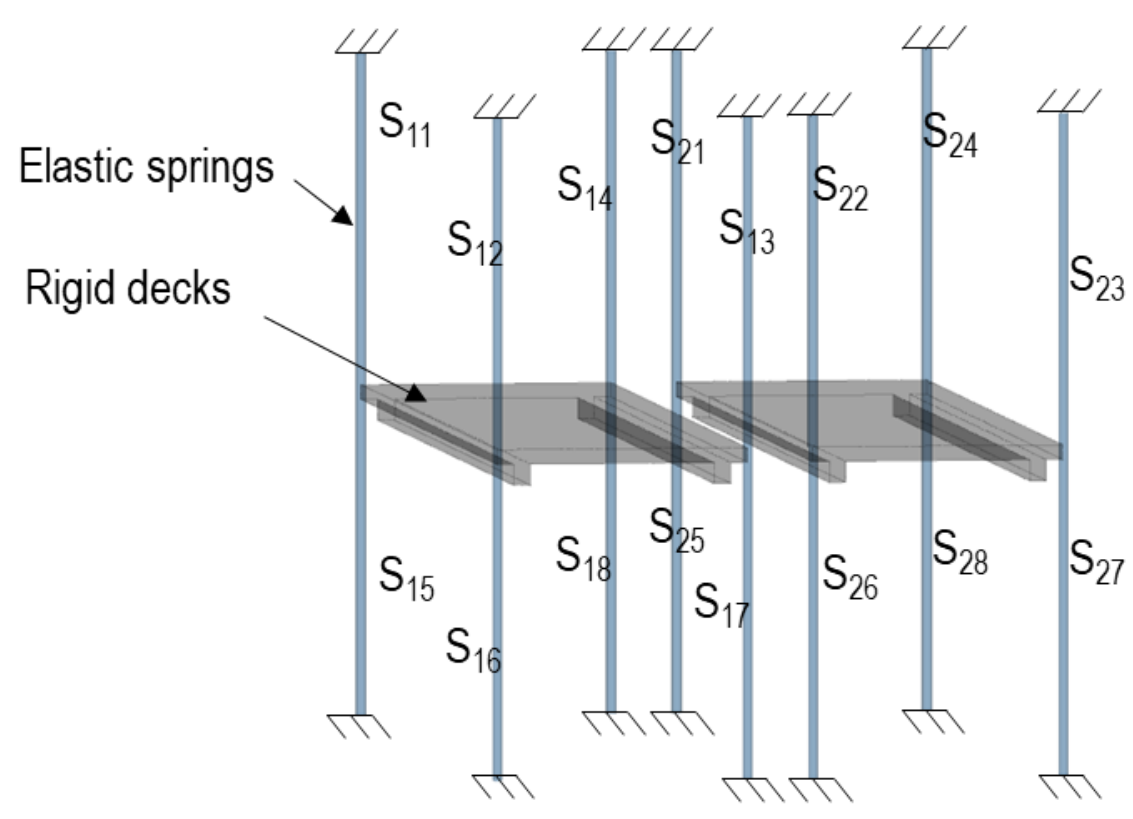

Figure 5-1: Twin deck model on elastic springs 
(a)

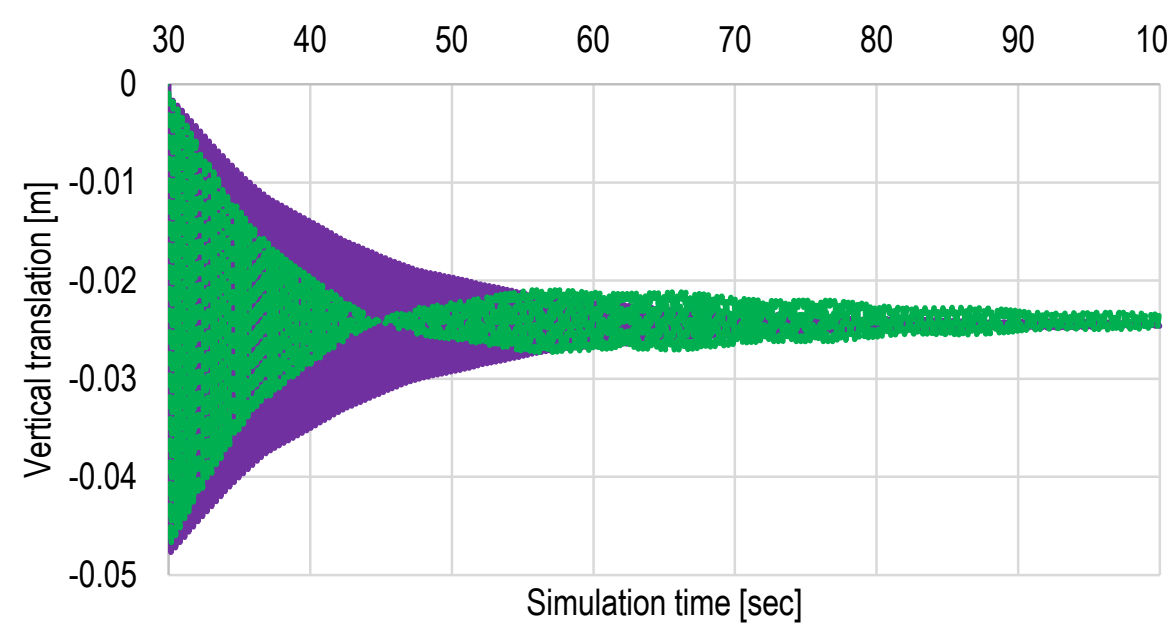

(b)

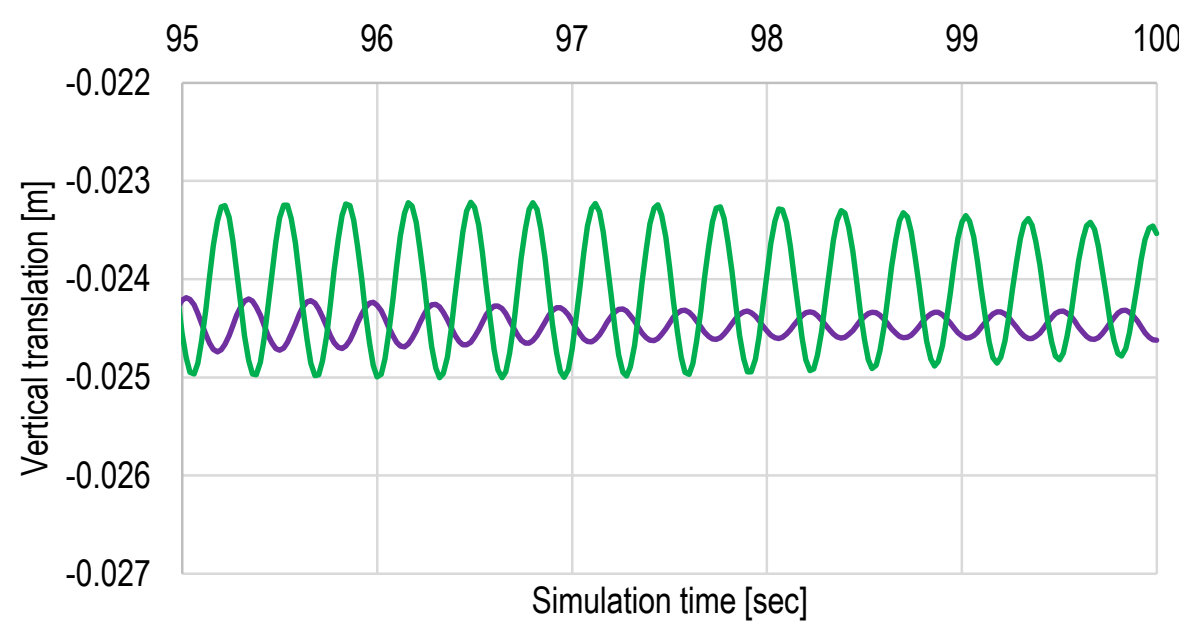

-Deck $1 \longrightarrow$ Deck 2

Figure 5-2: Vertical translations of the decks in time, (a) during the entire simulation, (b) during last 5 seconds of the simulation time. 


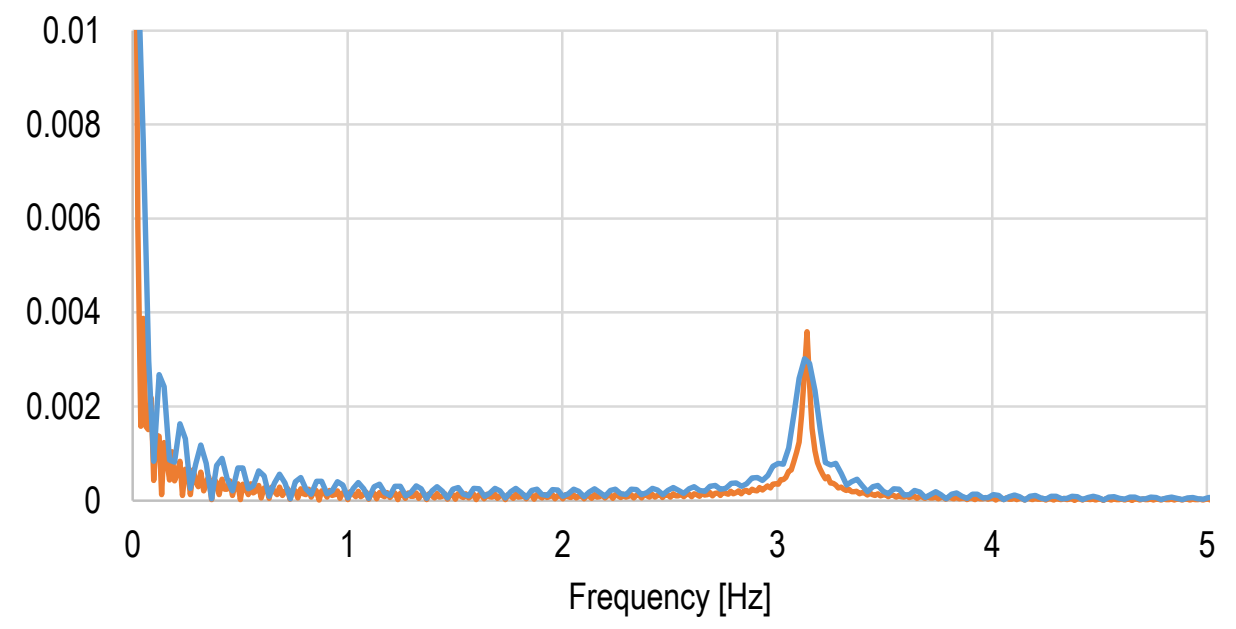

-Deck 1 Deck 2

Figure 5-3: Fast Fourier Transform of the vertical vibrations of the decks

(a)

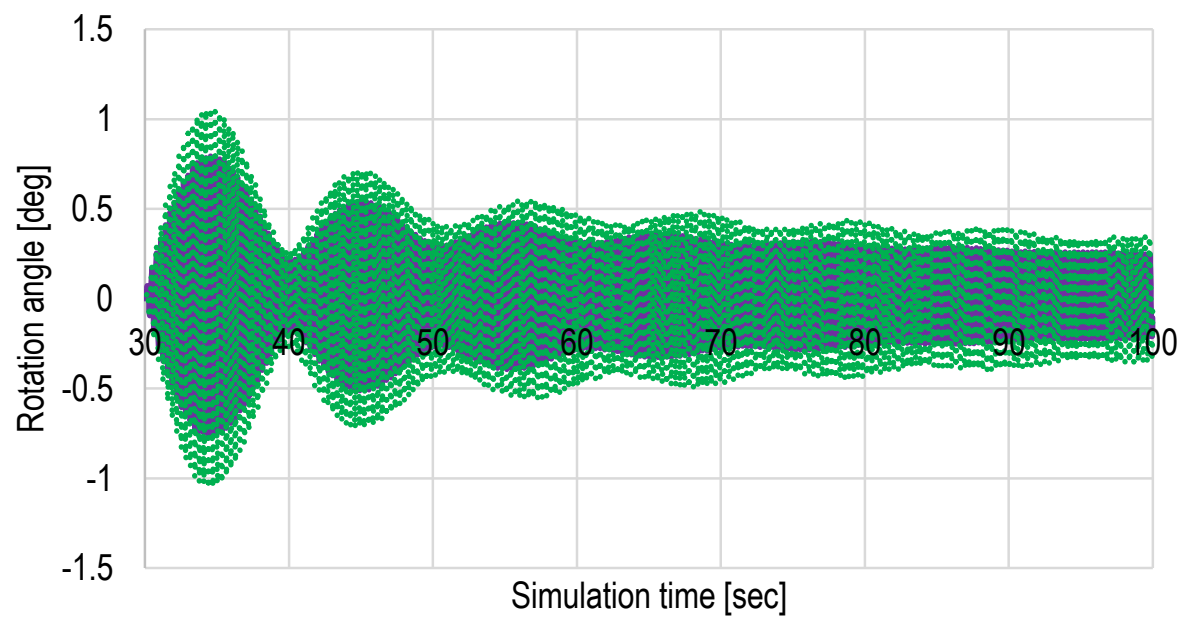

Deck 1

- Deck 2 
(b)

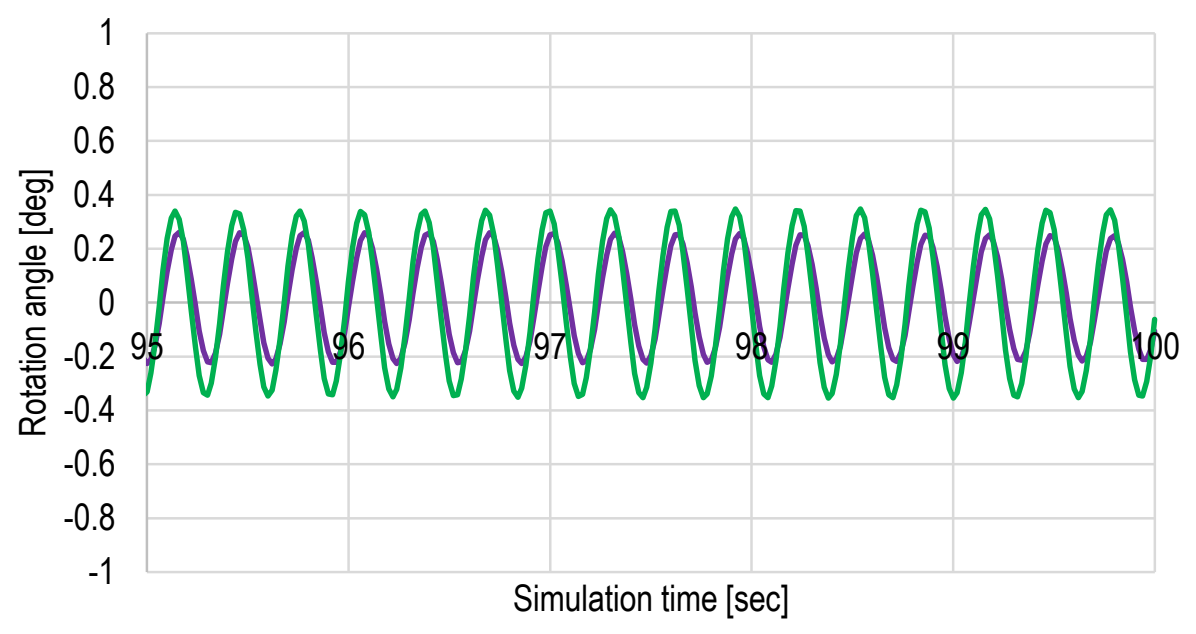

—Deck 1 -Deck 2

Figure 5-4: Rotations of the decks in time, (a) during the entire simulation, (b) during last 5 seconds of the simulation time

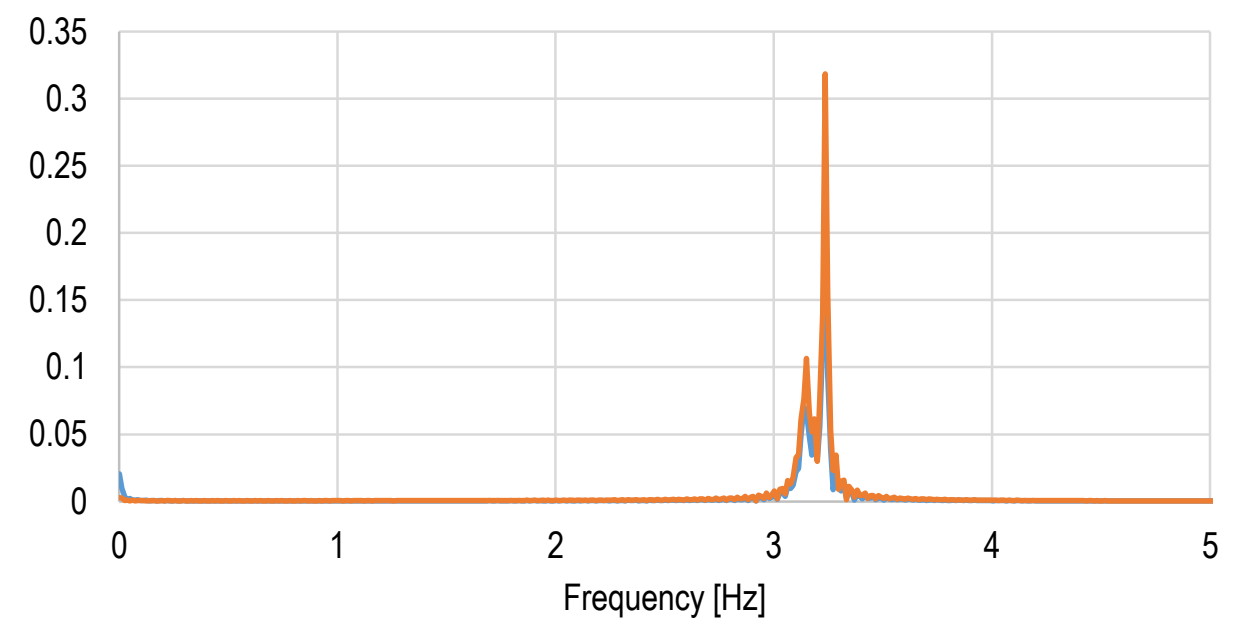

—Deck 1 -Deck 2

Figure 5-5: Fast Fourier Transform of the rotational vibrations of the decks 
(a)

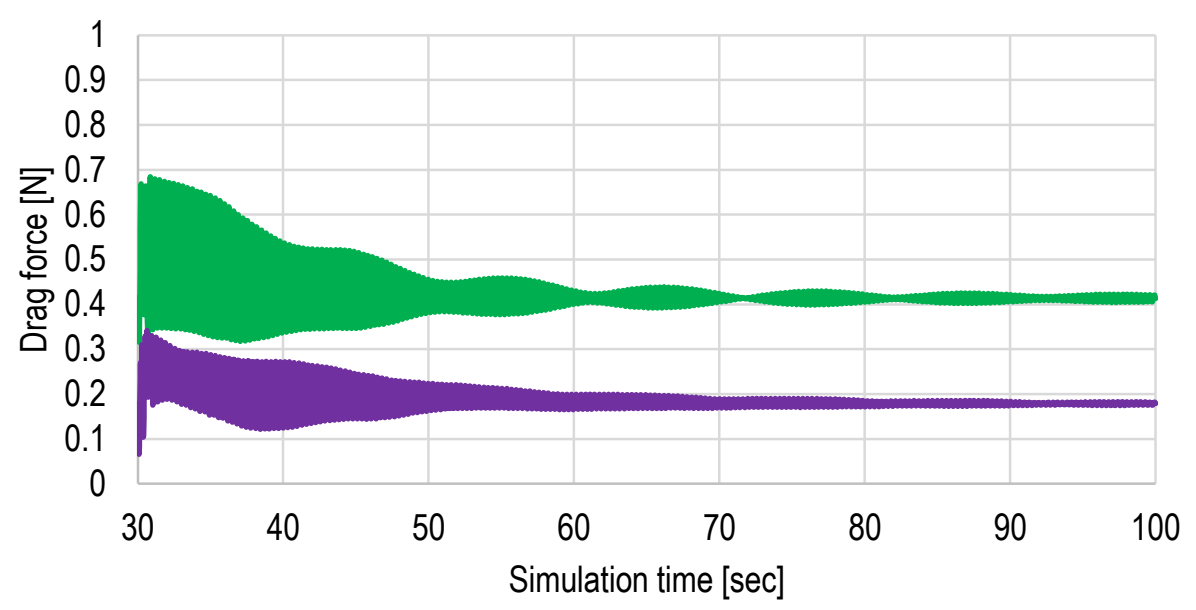

—Deck 1 -Deck 2

(b)

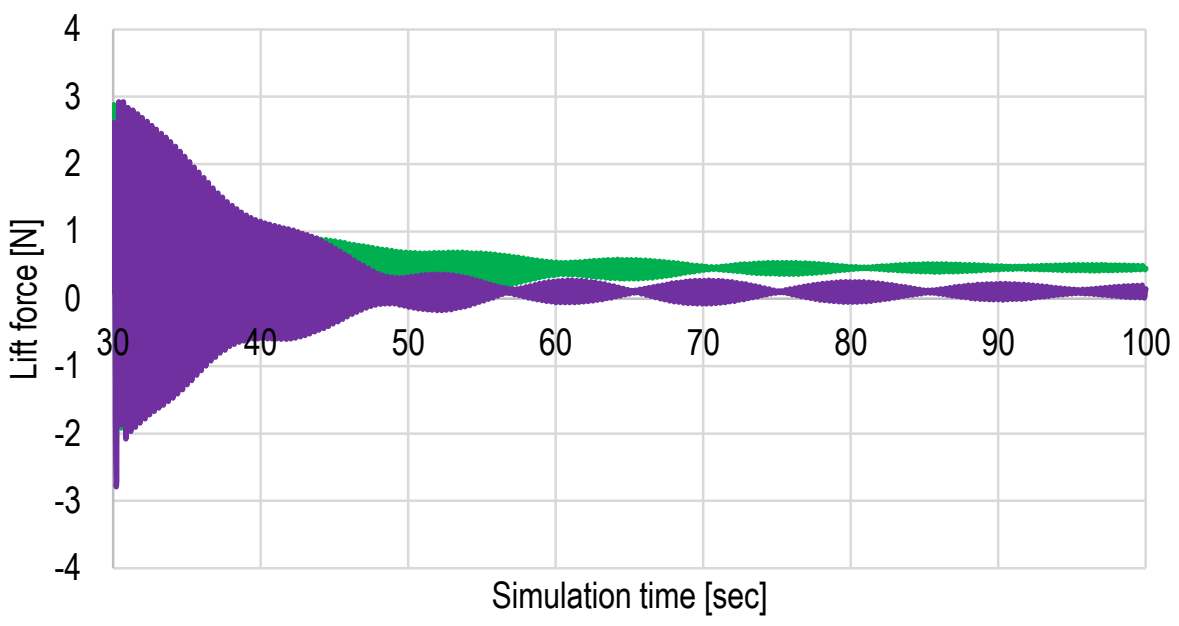

—Deck 1 -Deck 2

(c)

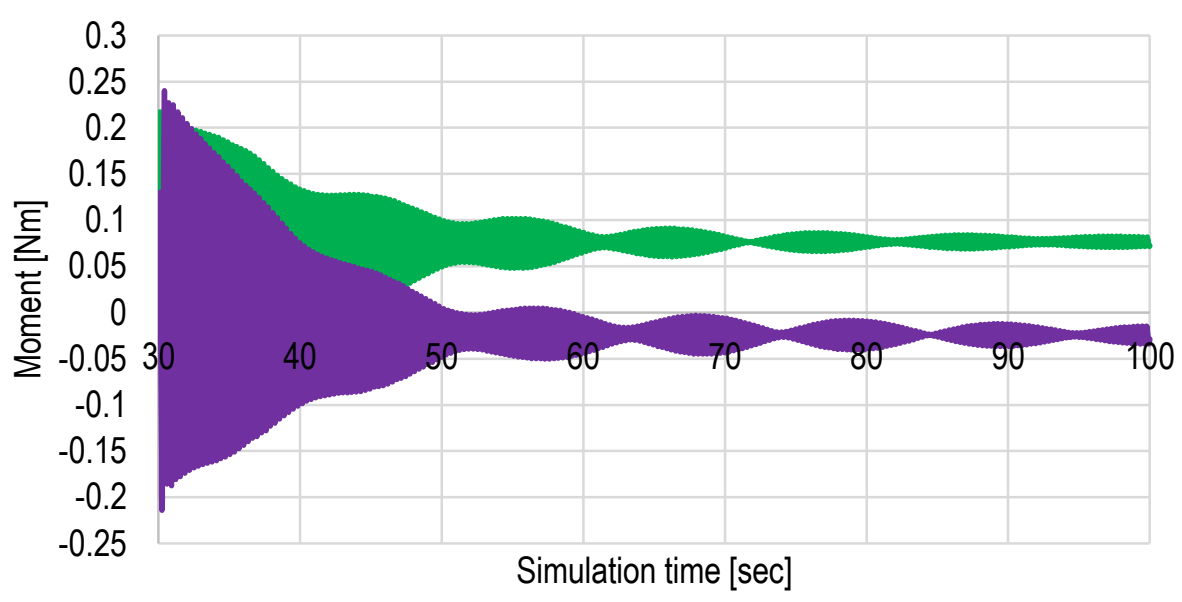

—Deck 1 -Deck 2

Figure 5-6: Forces acting on the decks in configuration 1, (a) drag, (b) lift and (c) moment 
Table 5-1. Comparison of drag force, lift force and pitch moment for a stationary twin deck and a twin deck suspended on elastic springs

\begin{tabular}{|c|c|c|c|c|c|c|}
\hline & \multicolumn{2}{|c|}{ Drag force $[\mathrm{N}]$} & \multicolumn{2}{c|}{ Lift force $[\mathrm{N}]$} & \multicolumn{2}{c|}{ Pitch moment [Nm] } \\
\hline & dynamic & stationary & dynamic & stationary & dynamic & stationary \\
\hline $\begin{array}{c}\text { upstream } \\
\text { deck }\end{array}$ & 0.414 & 0.413 & 0.462 & 0.455 & 0.077 & 0.086 \\
\hline $\begin{array}{c}\text { downstream } \\
\text { deck }\end{array}$ & 0.179 & 0.178 & 0.107 & 0.108 & -0.024 & -0.02 \\
\hline
\end{tabular}

Table 5-2: Velocity vector field on the middle plane in consecutive time steps

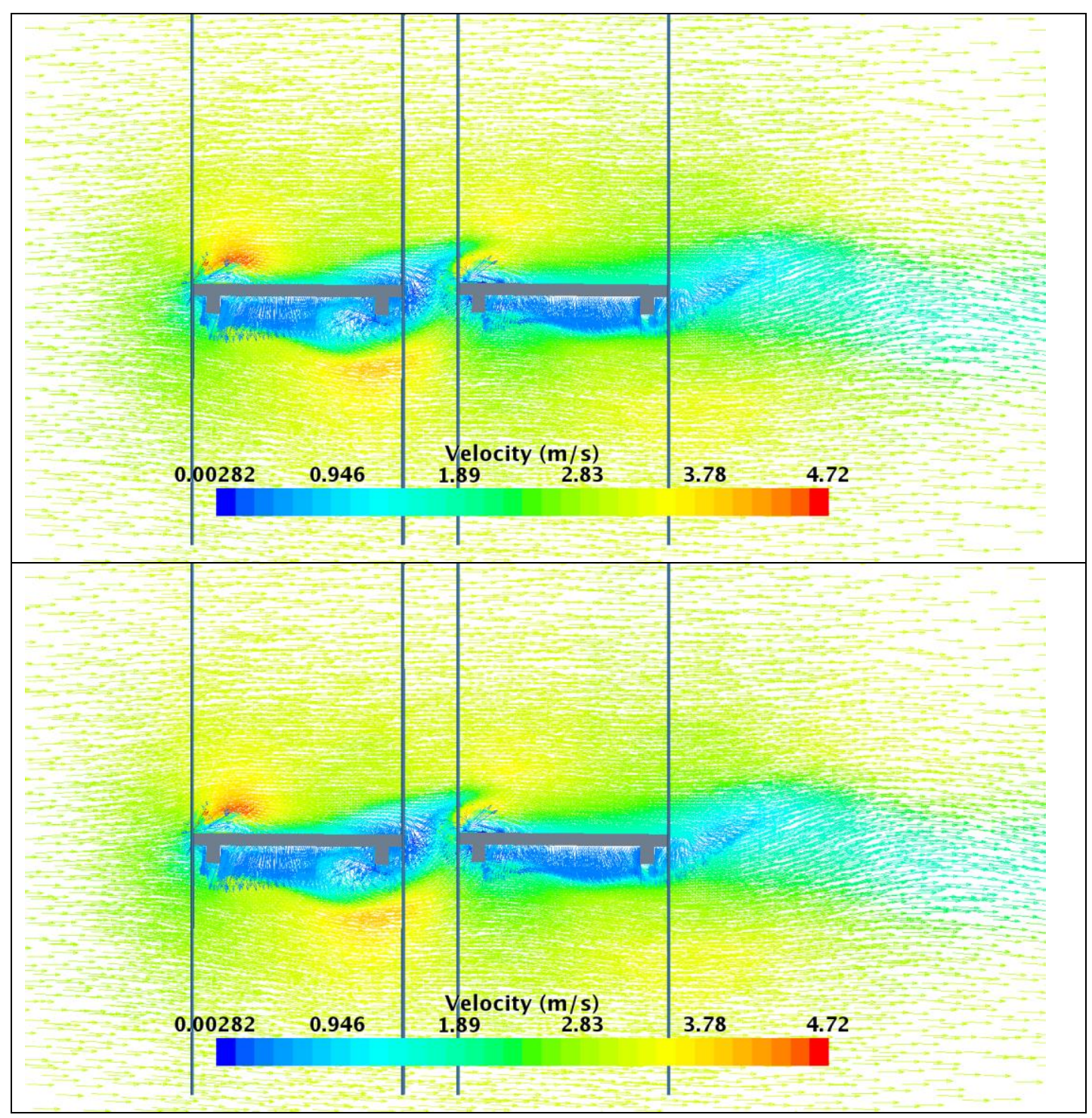




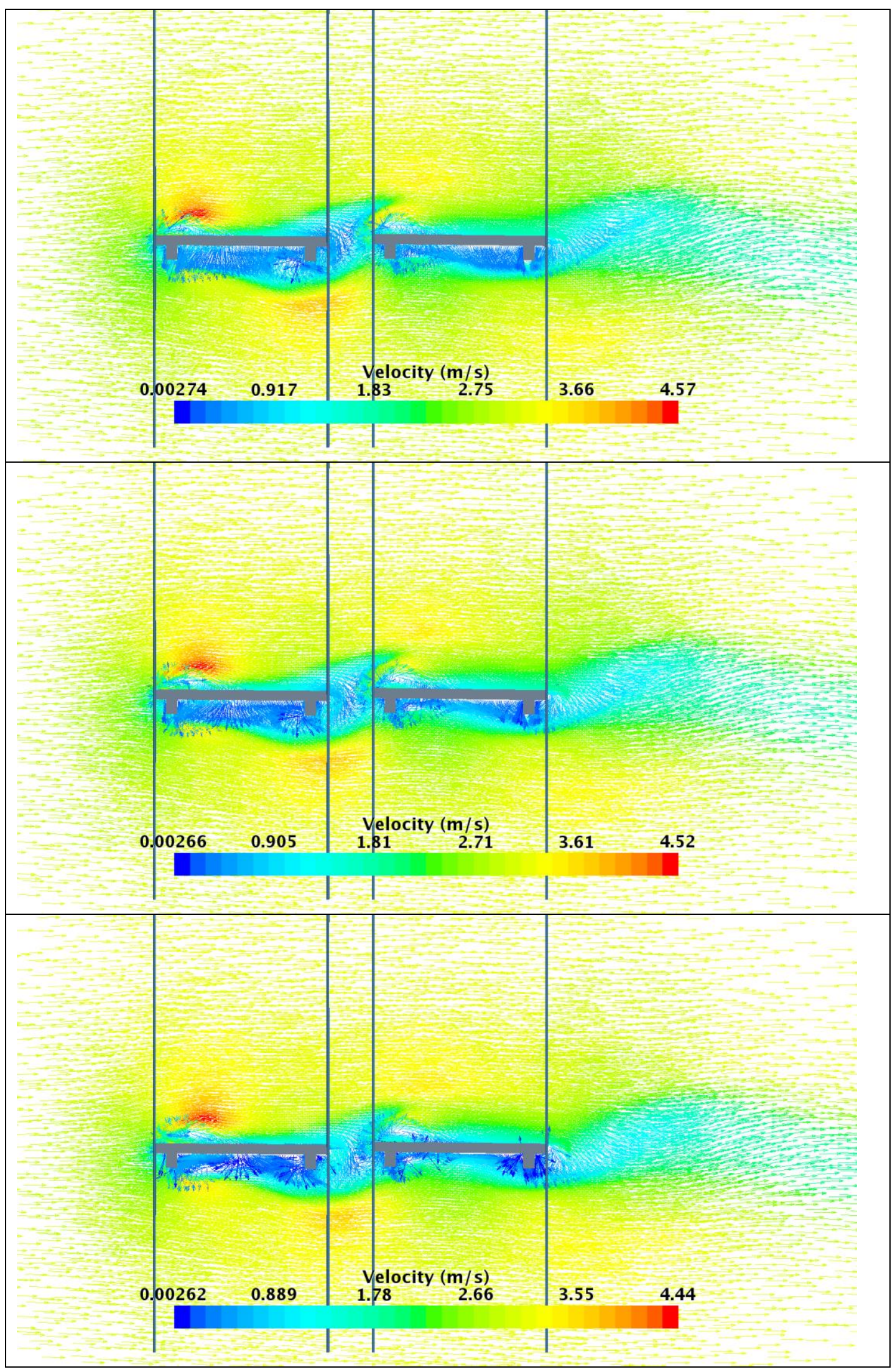

Investigation of Aerodynamic Interference between Twin Deck Bridges

Page $\mid 45$ 


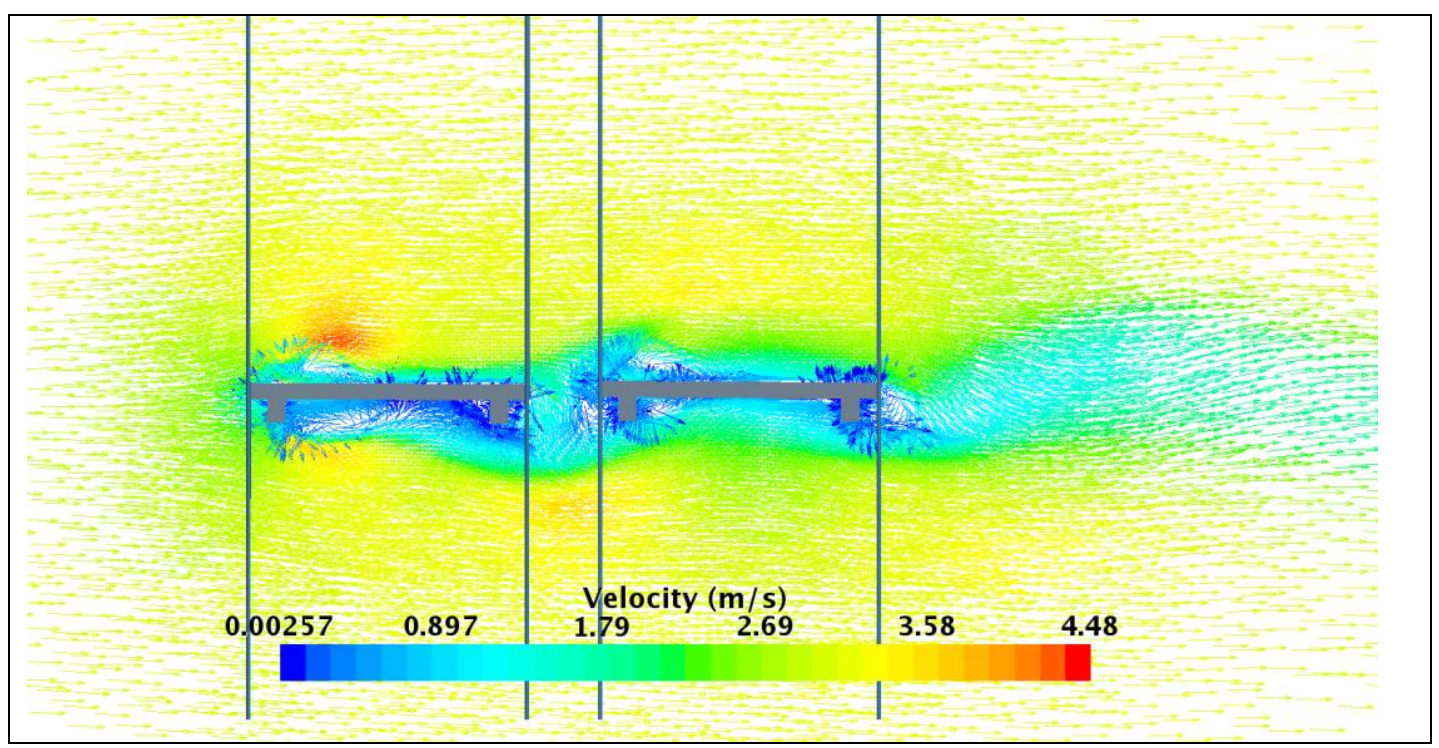

Table 5-3: Velocity streamlines in consecutive time steps

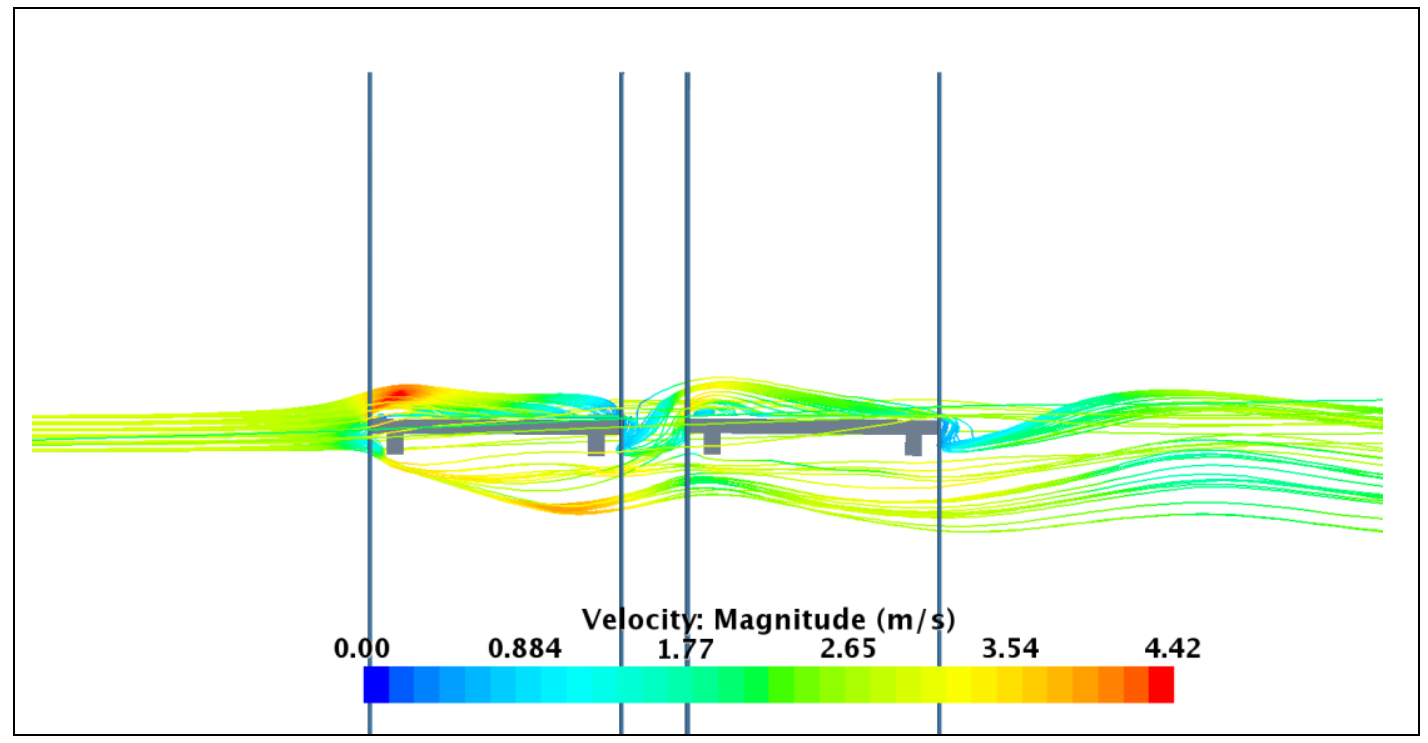




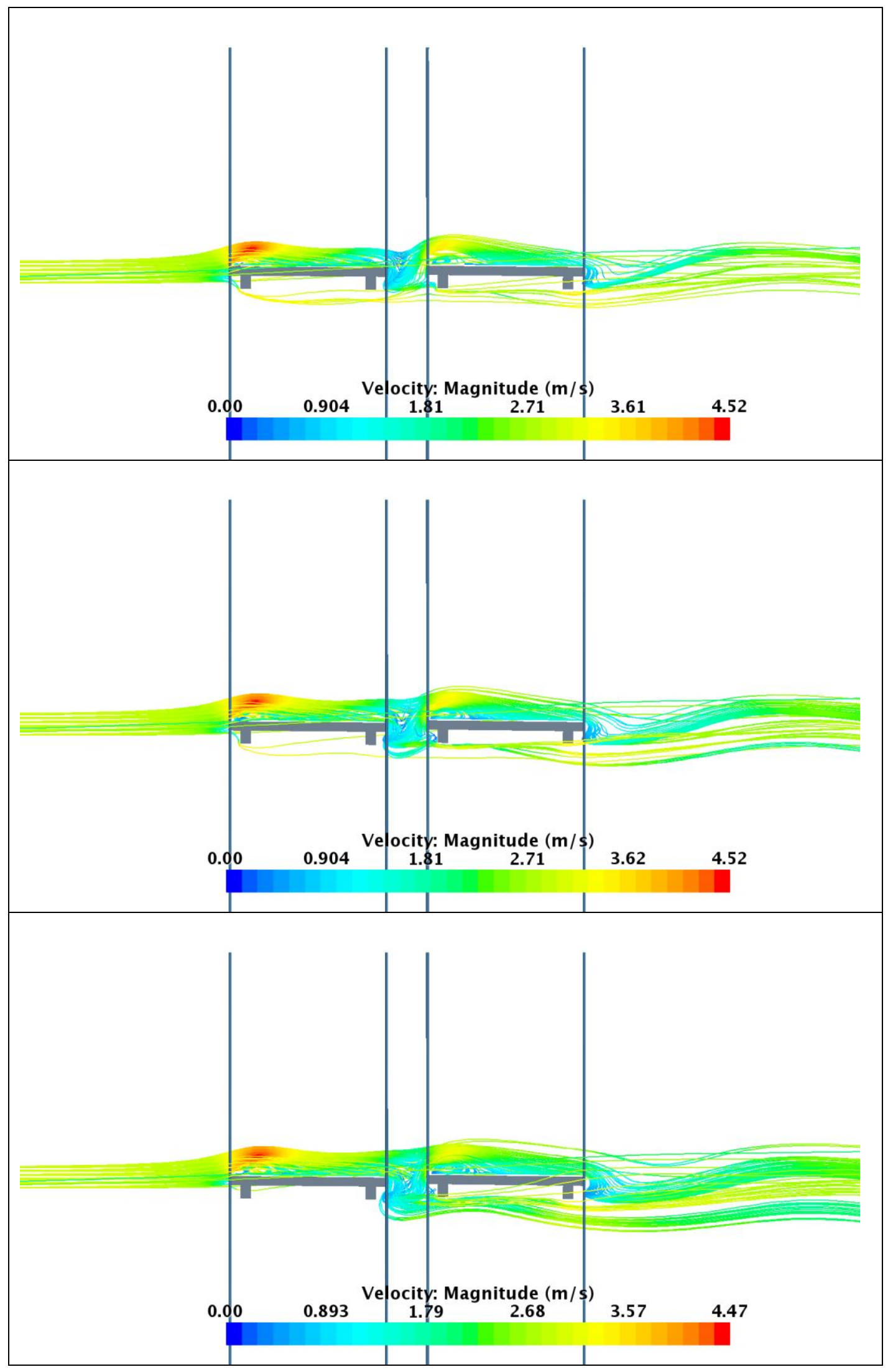

Investigation of Aerodynamic Interference between Twin Deck Bridges

Page $\mid 47$ 


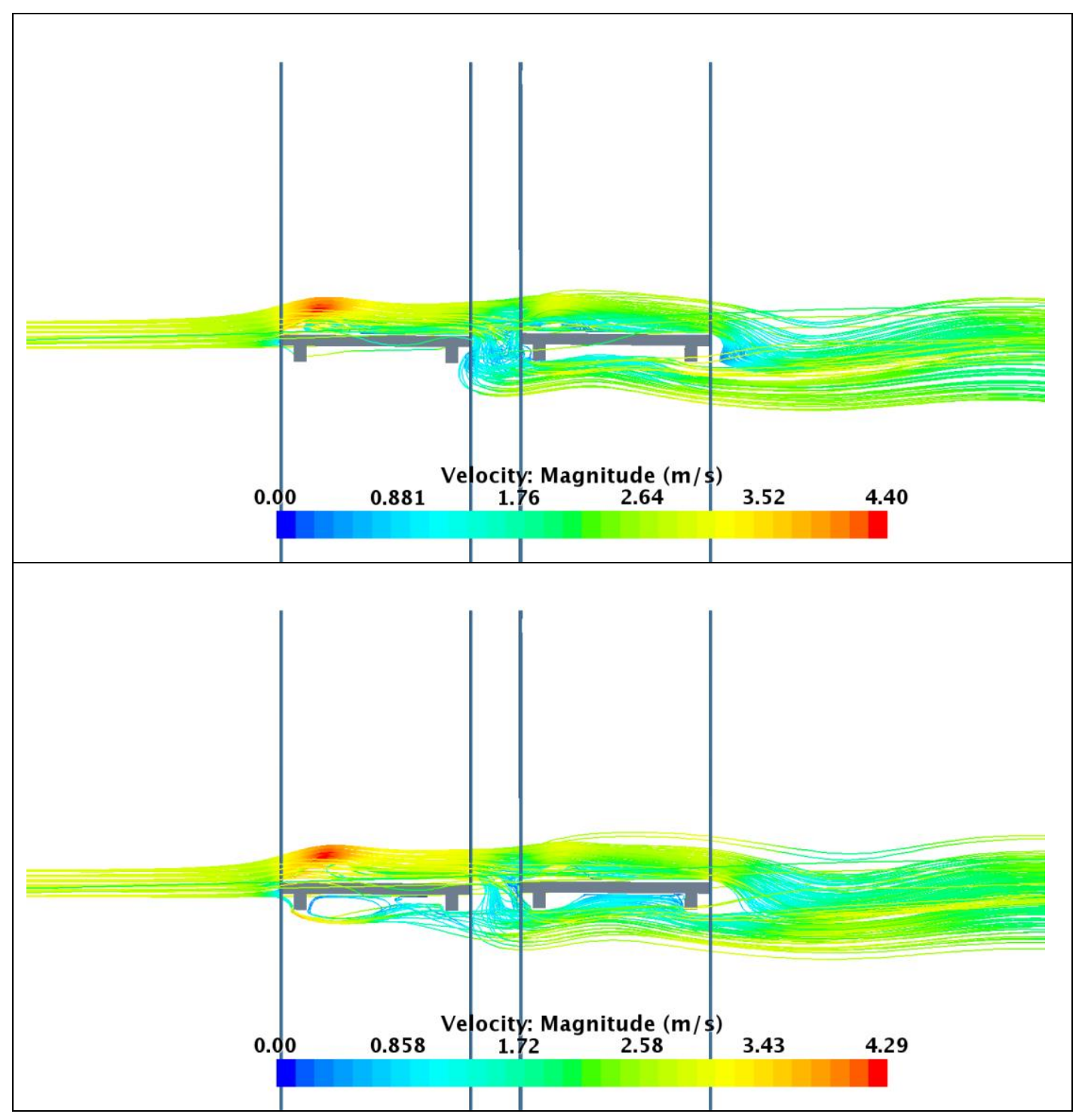

\subsection{Configuration 2: the upstream deck is suspended on springs, the downstream deck is constrained}

In this configuration only the upstream deck is suspended on elastic springs. At the beginning of the simulation it is positioned $0.0246 \mathrm{~m}$ higher than the downstream deck to take into account the elongation of elastic springs. After the springs are activated, it starts to vibrate in the vertical direction around the equilibrium position (which is aligned with the downstream deck position) and rotate along the center line of its cross-section. The initial amplitude of $4.8 \mathrm{~cm}$ quickly decreases to a millimeter and the rotations at the end of the simulation oscillate between -0.238 deg and $0.238 \mathrm{deg}$. Resultant drag forces, lift forces and moments for both decks are presented in Figure 5-7. 
(a)

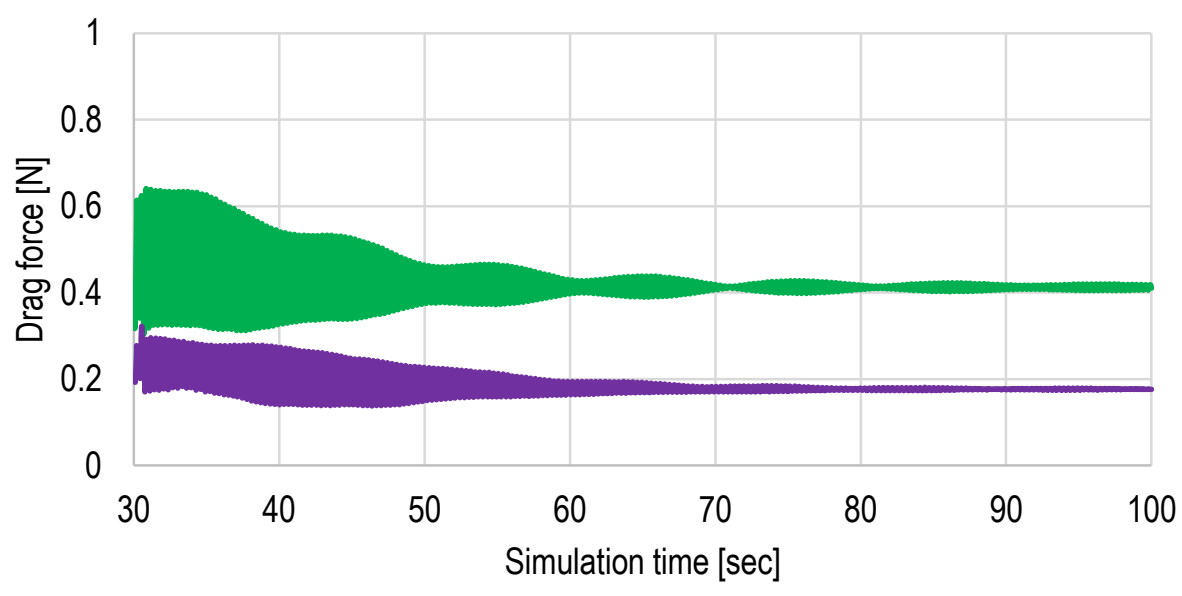

-Deck 1 Deck 2

(b)

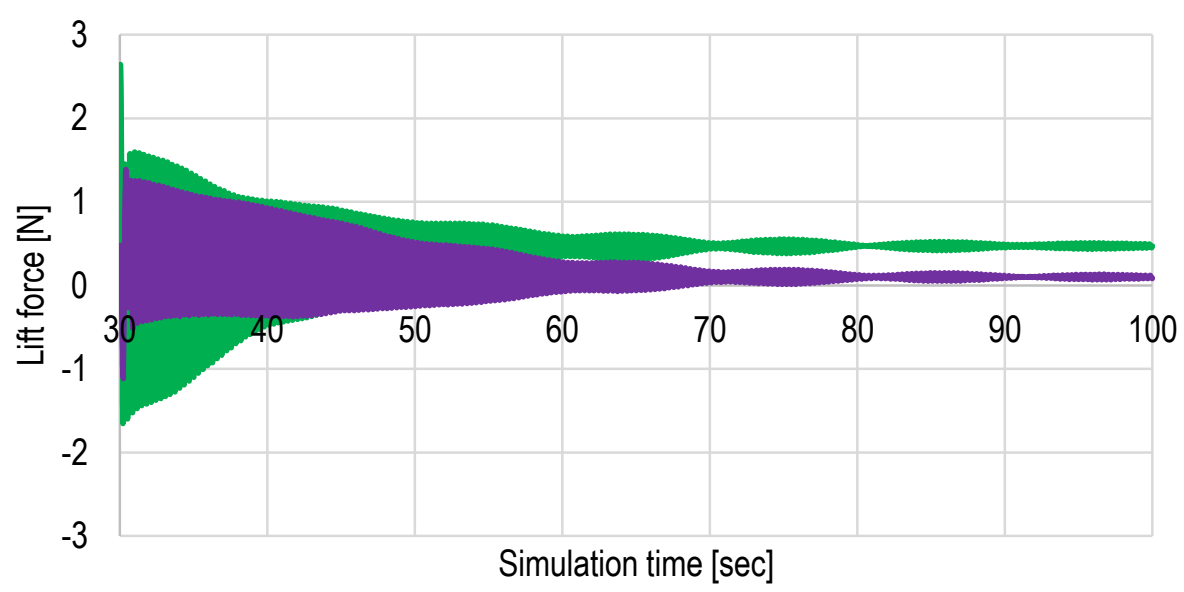

-Deck $1 \longrightarrow$ Deck 2

(c)

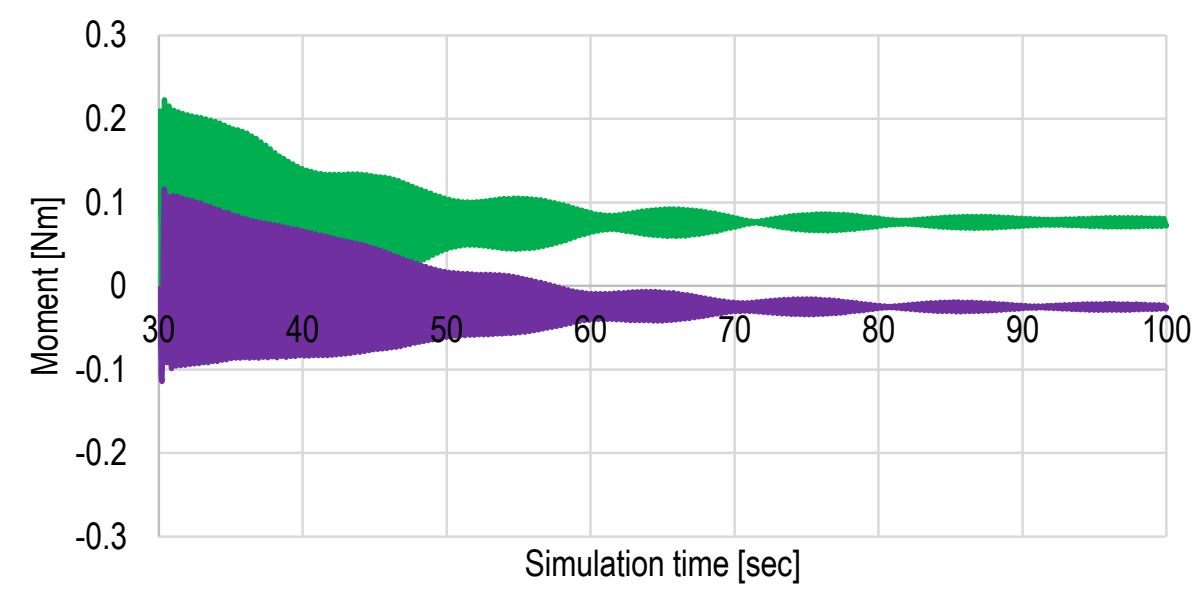

-Deck 1 -Deck 2

Figure 5-7: Forces and moment acting on the decks in configuration 2, (a) drag force, (b) lift force, (c) moment. 


\subsection{Configuration 3: the upstream deck is constrained, the downstream deck is suspended on springs}

The force components are also established for a third configuration, in which the upstream deck is constrained and the downstream deck is attached to springs, allowing for vertical motion and rotation about the deck center axis. The initial vertical position of the downstream deck is 0.0246 $\mathrm{m}$ higher than the upstream deck. After the springs are activated, it starts to vibrate in the vertical direction around the equilibrium position (which is aligned with the upstream deck position). Figure 5-8 summarizes the force and moment history during the simulation.

(a)

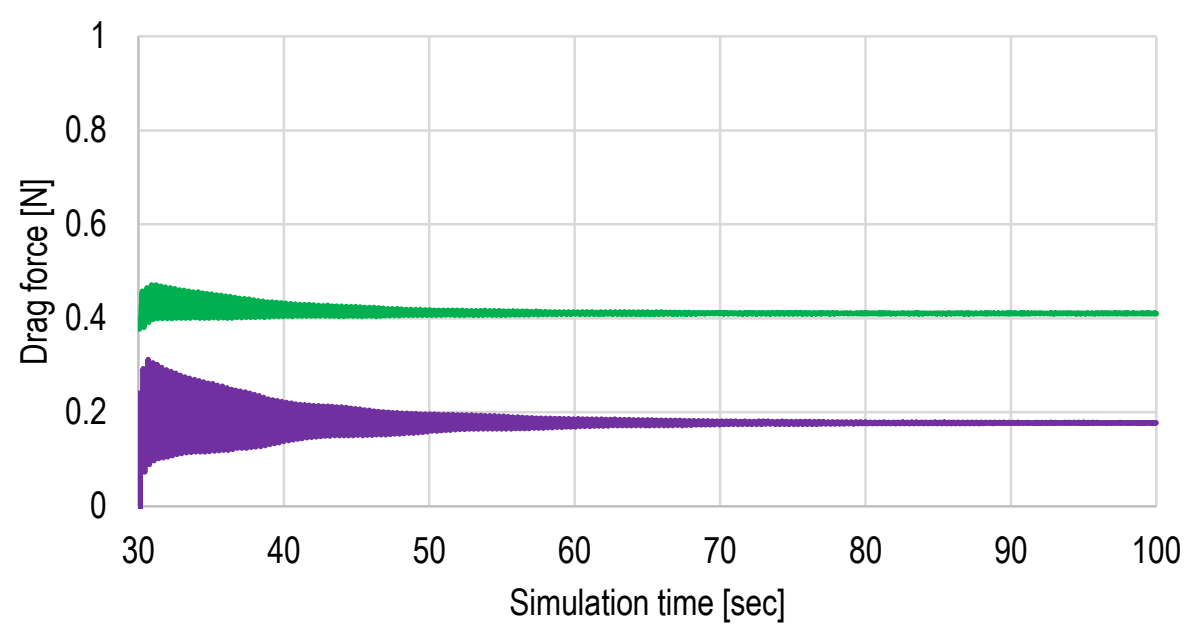

—Deck 1 -Deck 2

(b)

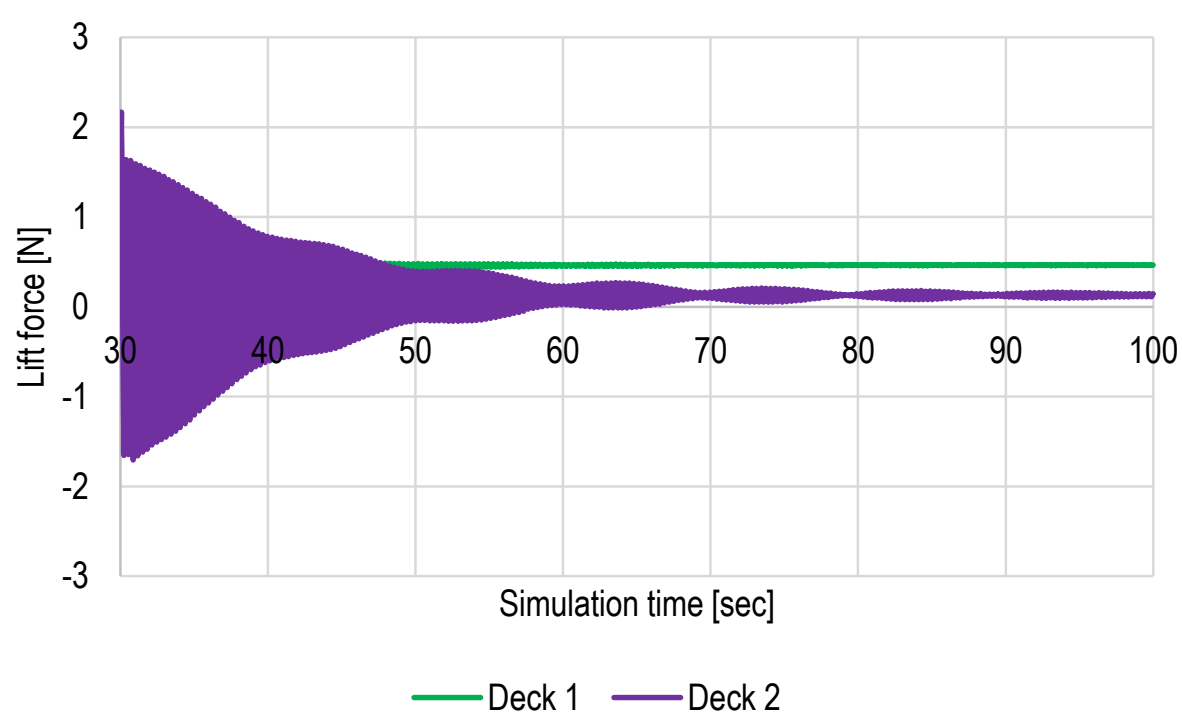


(c)

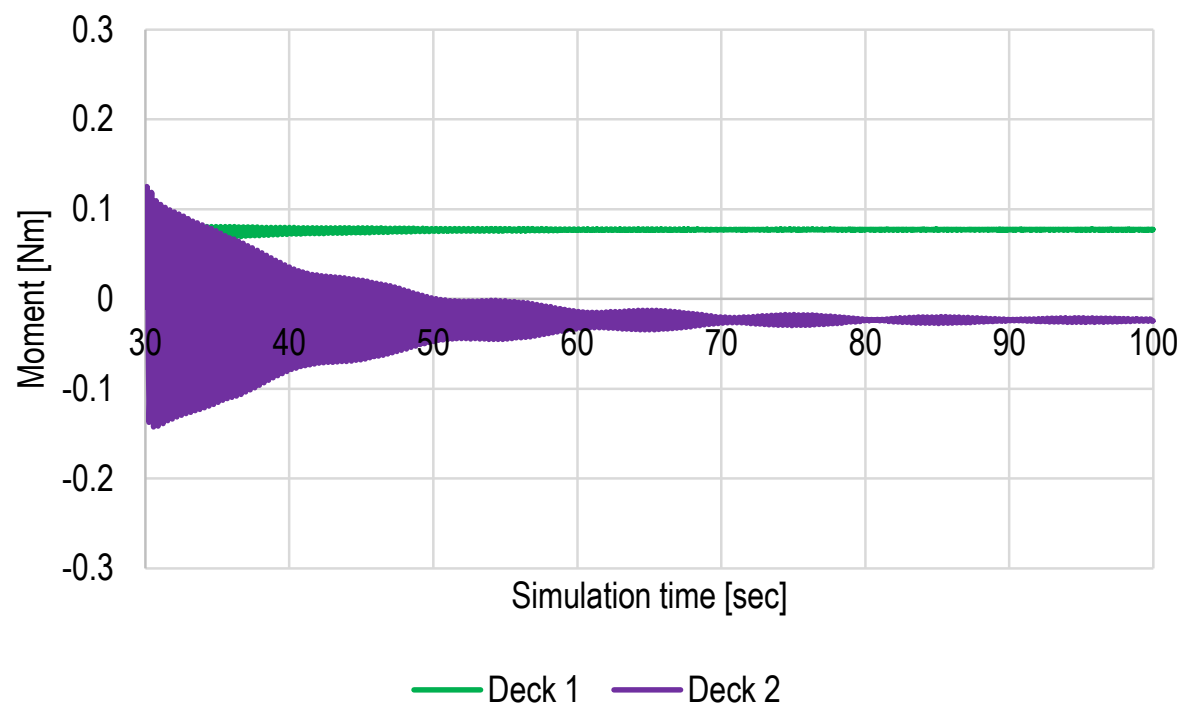

Figure 5-8: Forces and moment acting on the decks in configuration 3, (a) drag force, (b) lift force, (c) moment

\subsection{Comparison of results for configurations 1,2 , and 3}

Figure 5-9 and Figure 5-10 compare the force and moment between the stationary model and the time averaged force values obtained from three DFBI models. The averaging is done over the last ten seconds of the simulation, meaning between 90 seconds and 100 seconds of simulation time. The upstream deck does not experience significant changes in drag forces. The difference in drag forces is small, the differences are less than $2 \%$. The difference in lift forces is up to $4 \%$. The differences in pitch moments reach a maximum of $12 \%$.

The drag force acting on the downstream deck changes the value by only $1 \%$. The difference in the lift force and moment acting on the downstream deck is more significant. The lift force changes between $-7 \%$ (in configuration 2) to $19 \%$ (configuration 3 ). The biggest difference in pitch moment is between the base case and configuration 2, where pitch moment increased by $25 \%$. 
(a)

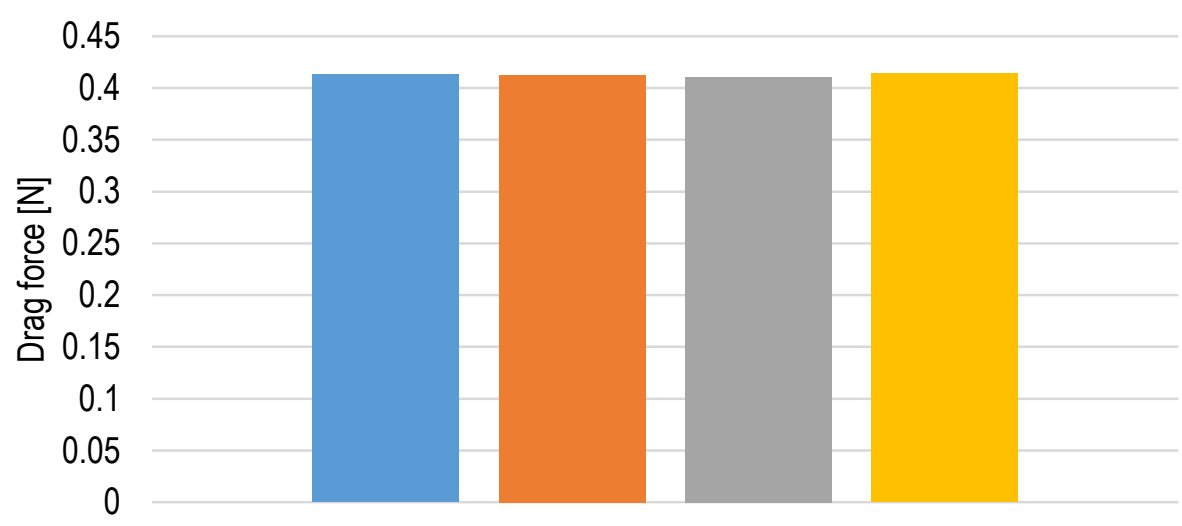

घ stationary decks $\quad$ configuration $1 \quad$ a configuration $2 \square$ configuration 3

(b)

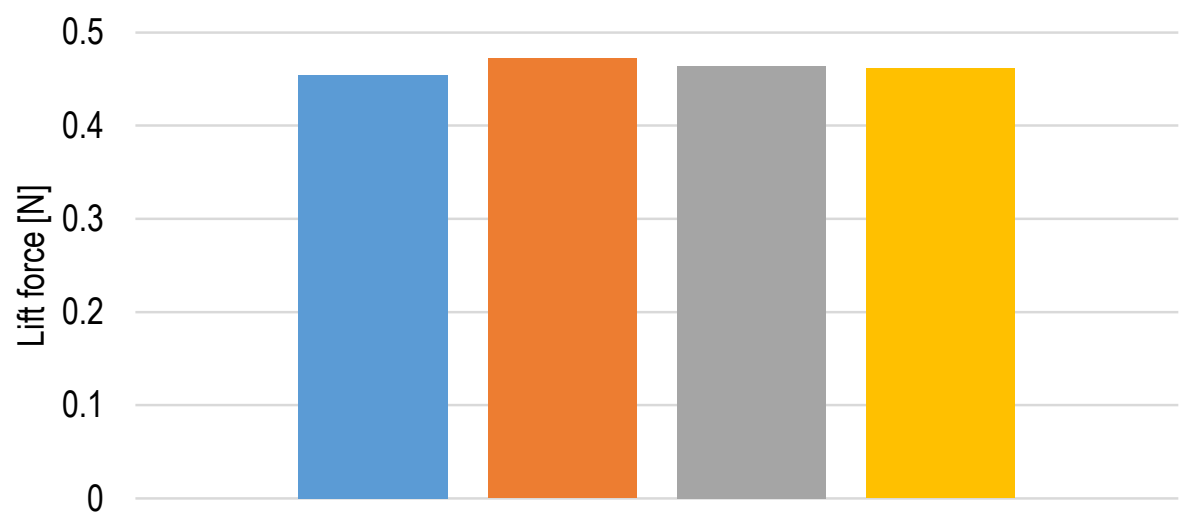

घstationary decks $\quad$ configuration $1 \quad$ w configuration $2 \approx$ configuration 3

(c)

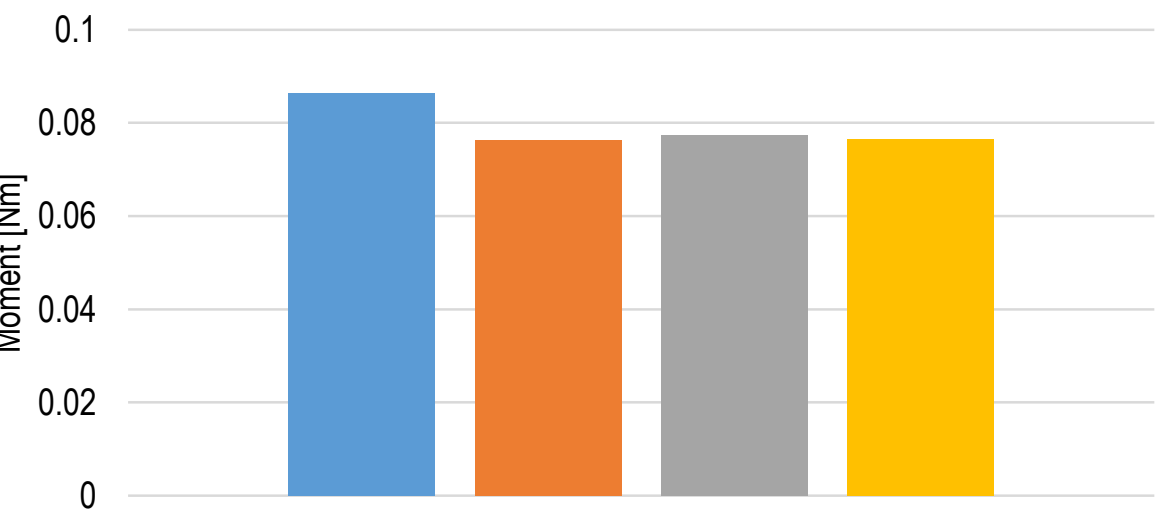

- stationary decks $\quad$ configuration $1 \quad$ m configuration $2 \square$ configuration 3

Figure 5-9: Comparison of force values acting on deck 1 at different configurations 
(a)

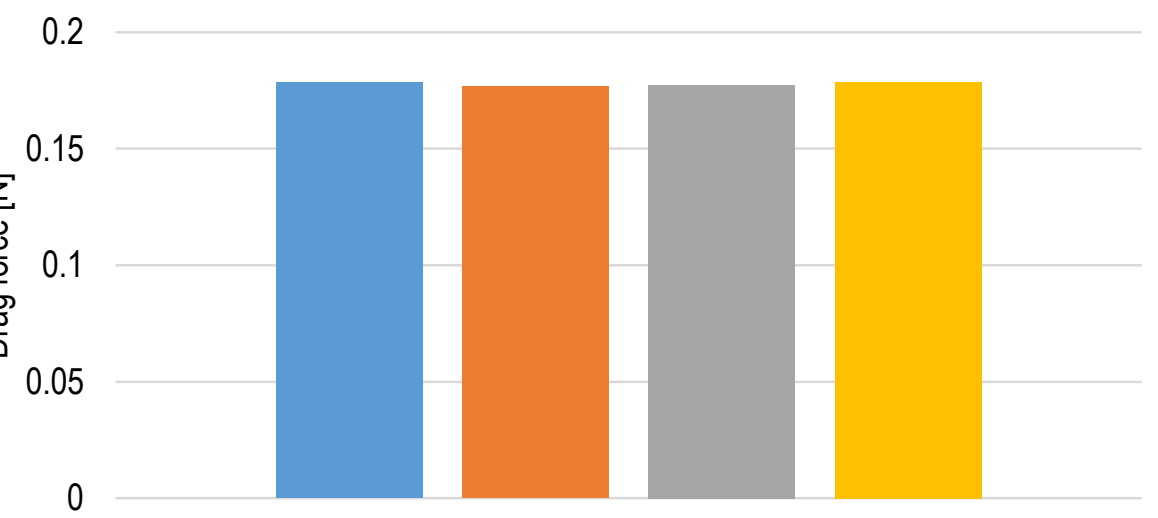

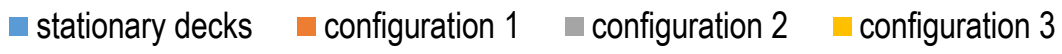

(b)

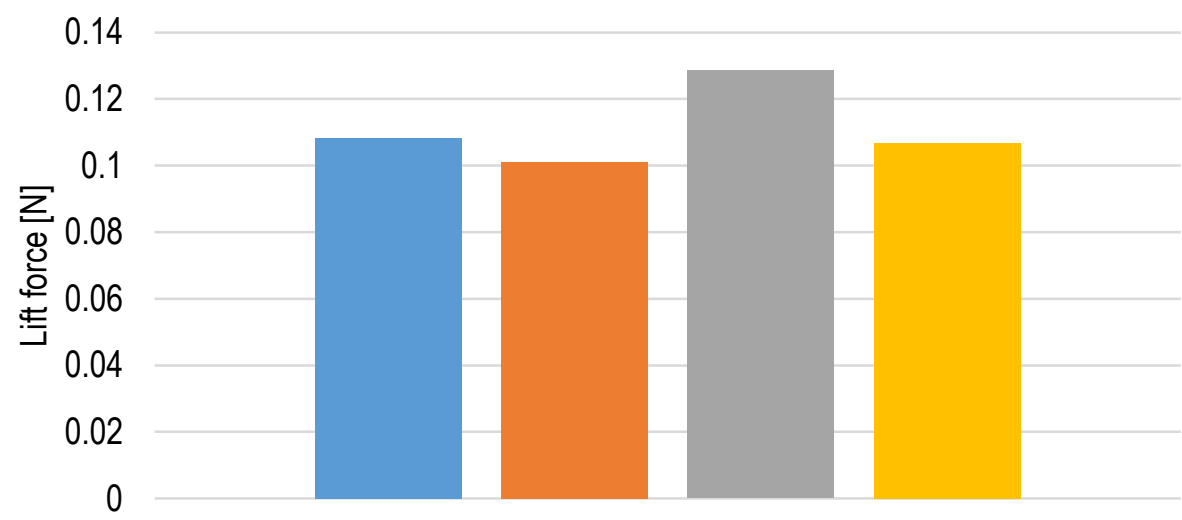

घ stationary decks $\square$ configuration $1 \backsim$ configuration $2 \square$ configuration 3

(c)

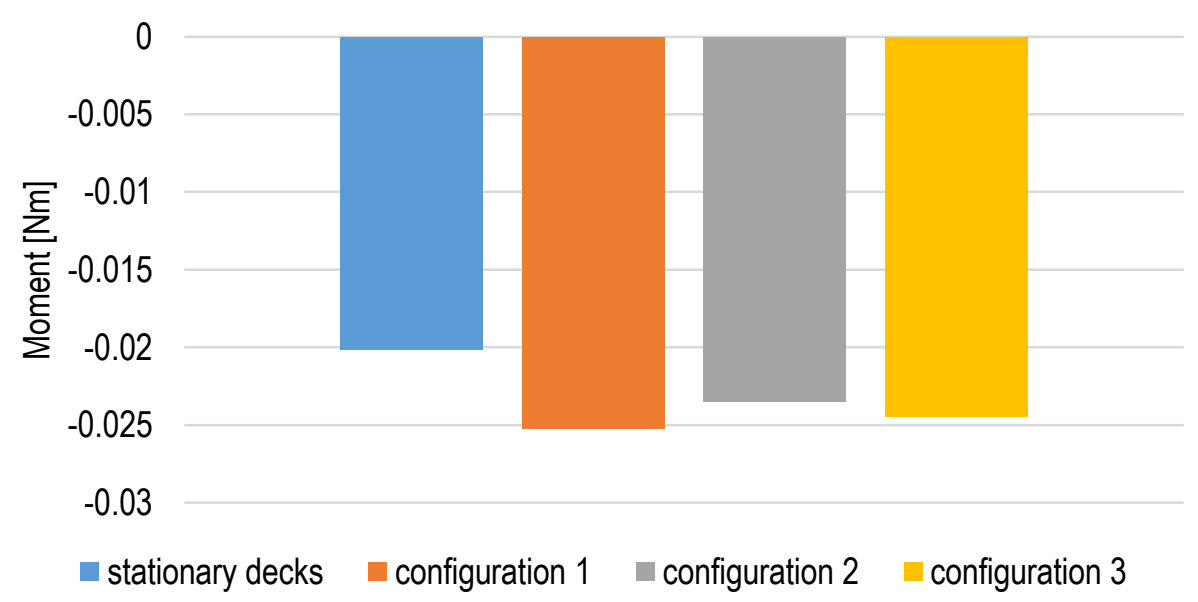

Figure 5-10: Comparison of force values acting on deck 2 at different configurations 


\section{Validation of the CFD modeling approach}

A study of the aerodynamic characteristics of the Stonecutters Bridge, described in chapter 1, is used as a validation reference of the modeling approach for analyzing interference between the decks of twin-deck bridges. The model was solved using unsteady Reynolds Averaged NavierStokes equations with a k-epsilon turbulence model and a trimmed cell computational mesh.

The bridge cross section is presented in Figure 6-1 with full-scale dimensions. Figure 6-2 shows the adopted sign convention for the angle of attack, $\alpha$, and force components ( $F_{D}-$ drag force, $F_{L}$ - lift force, $M$ - pitch moment), as well as for the approach flow velocity $\mathrm{U}$. The main dimensions of the prototype are as follows: the total width of the twin deck is $B=53.3 \mathrm{~m}$, width of a single deck is $C=19.5 \mathrm{~m}$, and deck height is $H=3.93 \mathrm{~m}$.

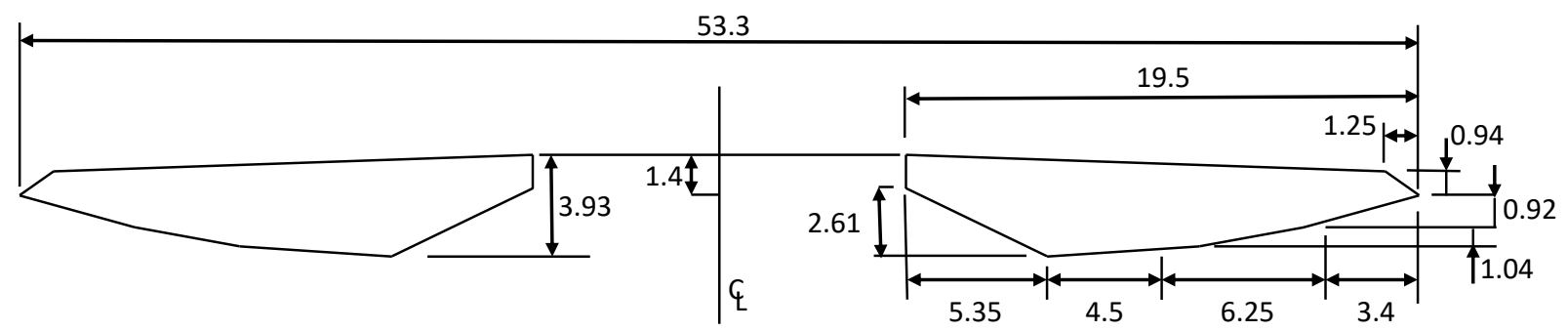

Figure 6-1: Stonecutters bridge cross-section. All dimensions are given in meters.

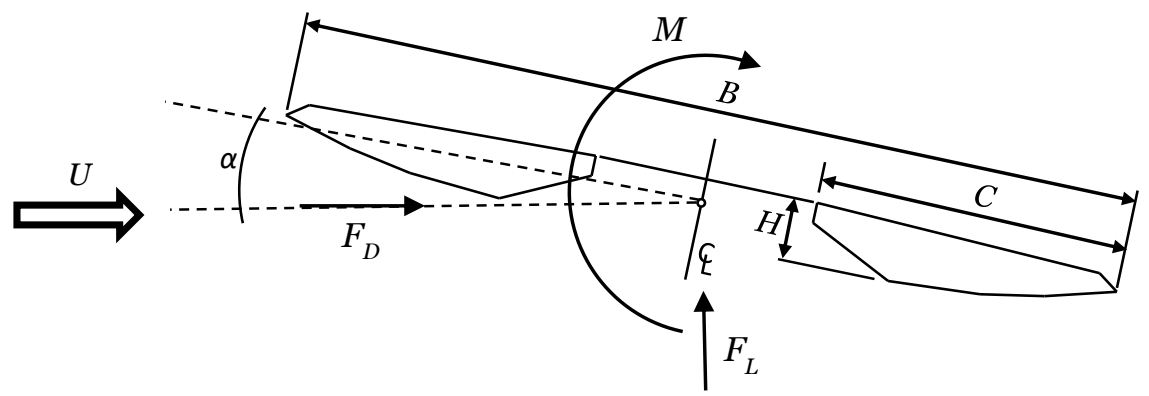

Figure 6-2: Sign convention

The study covers computation of static forces and corresponding coefficients (a) at varying flow velocity in parallel flow, (b) at varying direction of the approach flow for a chosen flow velocity, and (c) at velocities at which vortex induced vibrations were observed.

A set of computations was performed at a zero-degree angle of incidence and different approach flow velocities to assess the sensitivity of aerodynamic force coefficients to Reynolds number. The range of Reynolds number for these cases ranged from 15,000 to 62,000 (based on the height of the decks, $H$ ).

The force coefficients are calculated as follows:

$$
C_{D}=\frac{F_{D}}{0.5 \rho U^{2} B L}
$$




$$
\begin{aligned}
C_{L} & =\frac{F_{L}}{0.5 \rho U^{2} B L}, \\
C_{M} & =\frac{M}{0.5 \rho U^{2} B^{2} L^{2}},
\end{aligned}
$$

with $B$, the total width of the bridge, and taken as the characteristic length, $L$ is a unit length.

Figure 6-3 presents a plot of the sensitivity of the force coefficients to Reynolds number change at zero angle of attack. The drag and moment coefficients differ for the lowest Re, but overall they are independent of flow velocity. The lift coefficient goes asymptotic later than drag force and pitch moment. Its absolute value keeps increasing up to $\mathrm{Re}=47,000$, and tends to become constant for higher values of Re. These results are consistent with those reported in [22].

Figure 6-4, Figure 6-5, and Figure 6-6 illustrate the drag, lift and pitch moment coefficient obtained in CFD simulations and tests versus attack angles. They show a good agreement between the simulation and experimental results found in the literature [25], except for drag force and pitch moment at -10 degrees, and lift force and pitch moment at 10 degrees. This is not surprising because at larger angles of attack the variance of experimental measurements is higher.

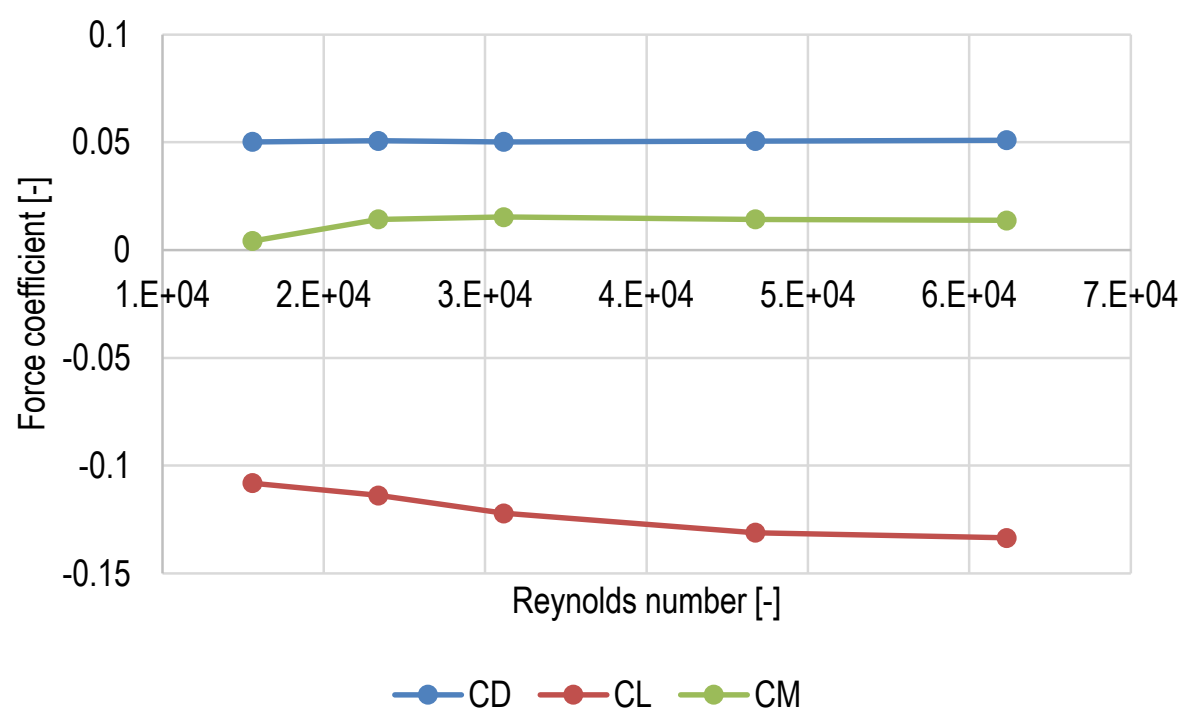

Figure 6-3: Sensitivity of force coefficients to Reynolds number 


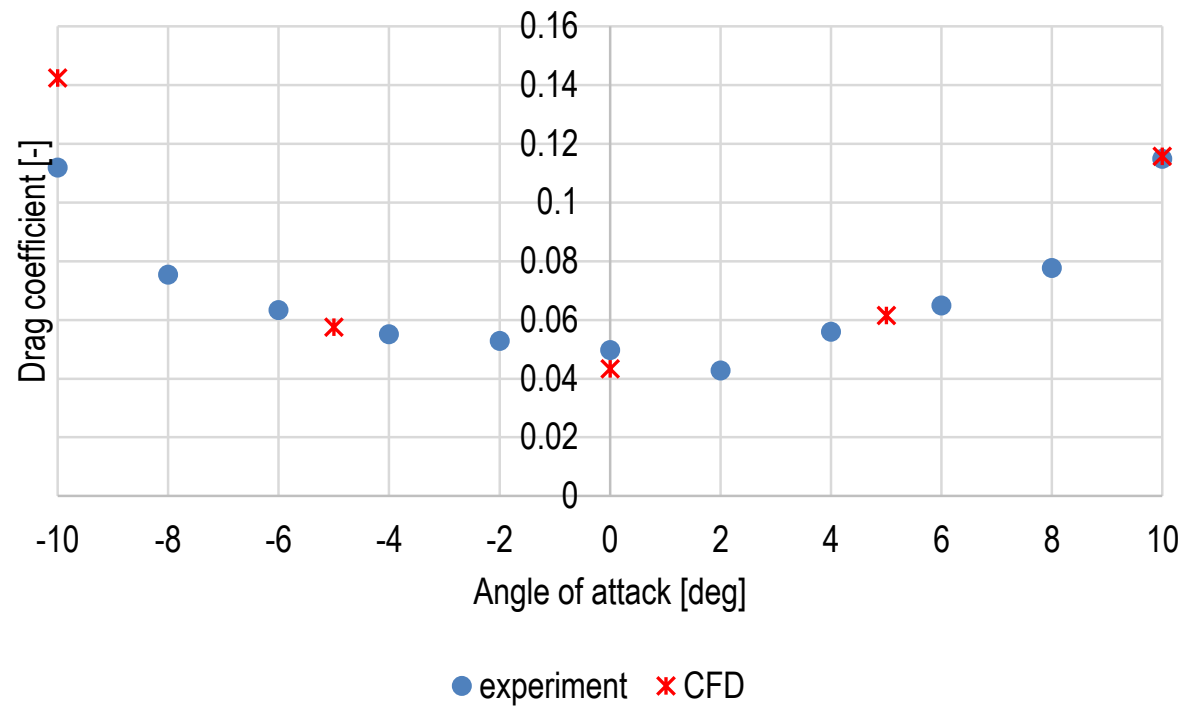

Figure 6-4: The drag coefficient at different angles of attack

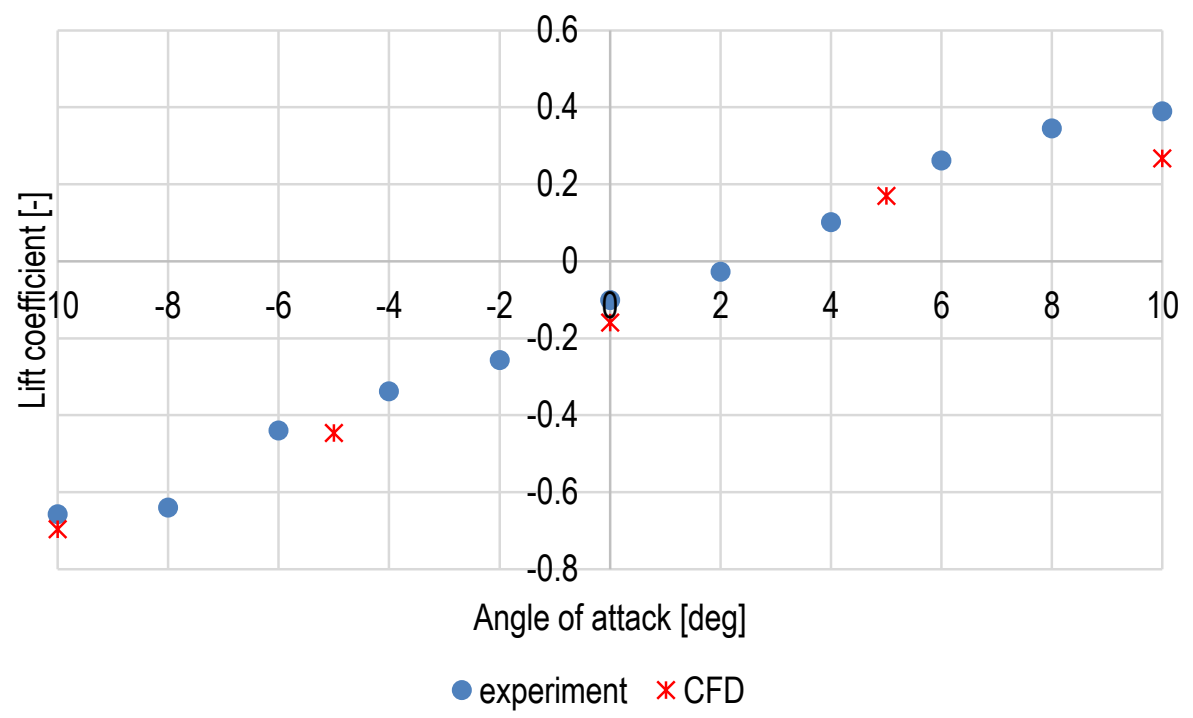

Figure 6-5: The lift coefficient at different angles of attack 


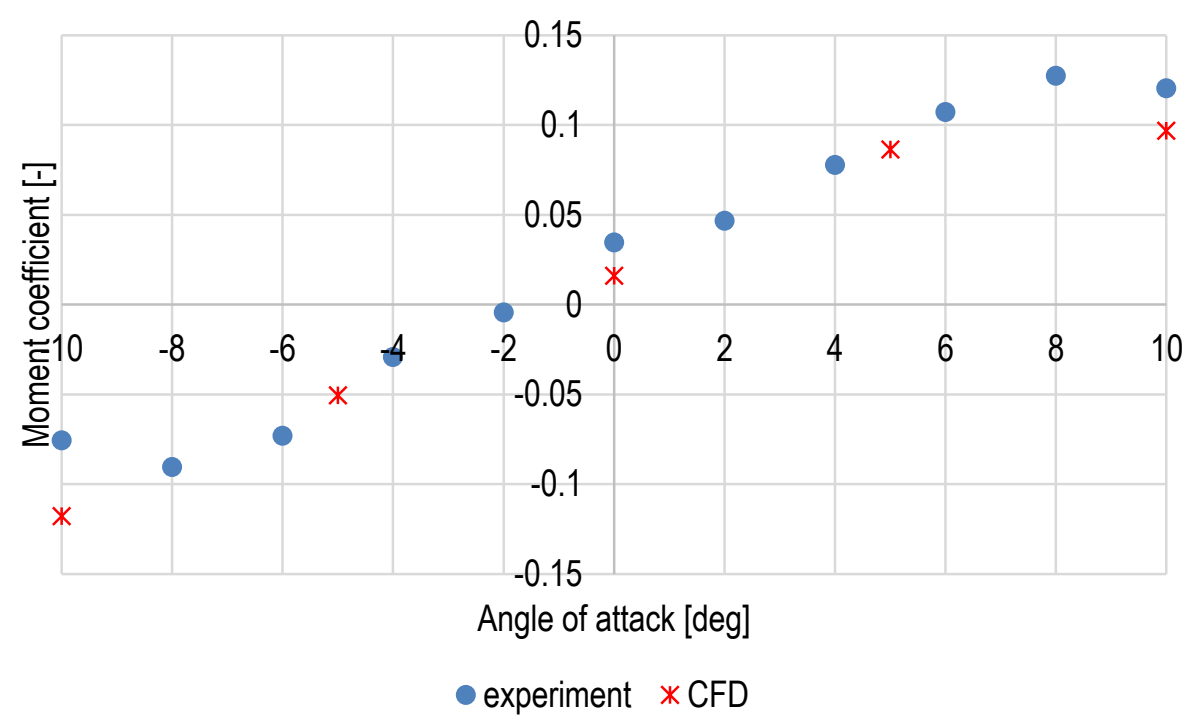

Figure 6-6: The pitch moment coefficient at different angles of attack

At the design stage of the Stonecutters Bridge it was discovered that the bridge was prone to vortex induced vibrations at certain values of Reynold numbers, which are $R e=12,000$ and $R e=91,000$ [25]. Velocity and vorticity fields are examined around the decks at these two values of Reynolds numbers. Also, the drag, lift force, and moment coefficients, as well as Strouhal number are calculated. Reference [18] presents, among others things, results of a comparison of Strouhal number values at different central gap widths.

The Strouhal number was established in two ways: from the spectra of its wake flow and integrated lift force. The corresponding values for the selected test configuration (selected gap, $14.3 \mathrm{~m}$ ) are equal to 0.23 and 0.27 , respectively.

Figure 6-7 presents the velocity fields and Figure 6-8 presents the vorticity fields on a vertical plane cut through the bridge decks at $\mathrm{Re}=12,000$ and $\mathrm{Re}=91,000$ respectively. A clear trail of twin vortices shows behind the decks at $\mathrm{Re}=12$,000. For the higher Re, the oscillations are not as pronounced. In both cases, the downstream deck is located in the wake of the upstream deck and is subjected to the oscillating vortices separating from the upstream deck. 
(a)

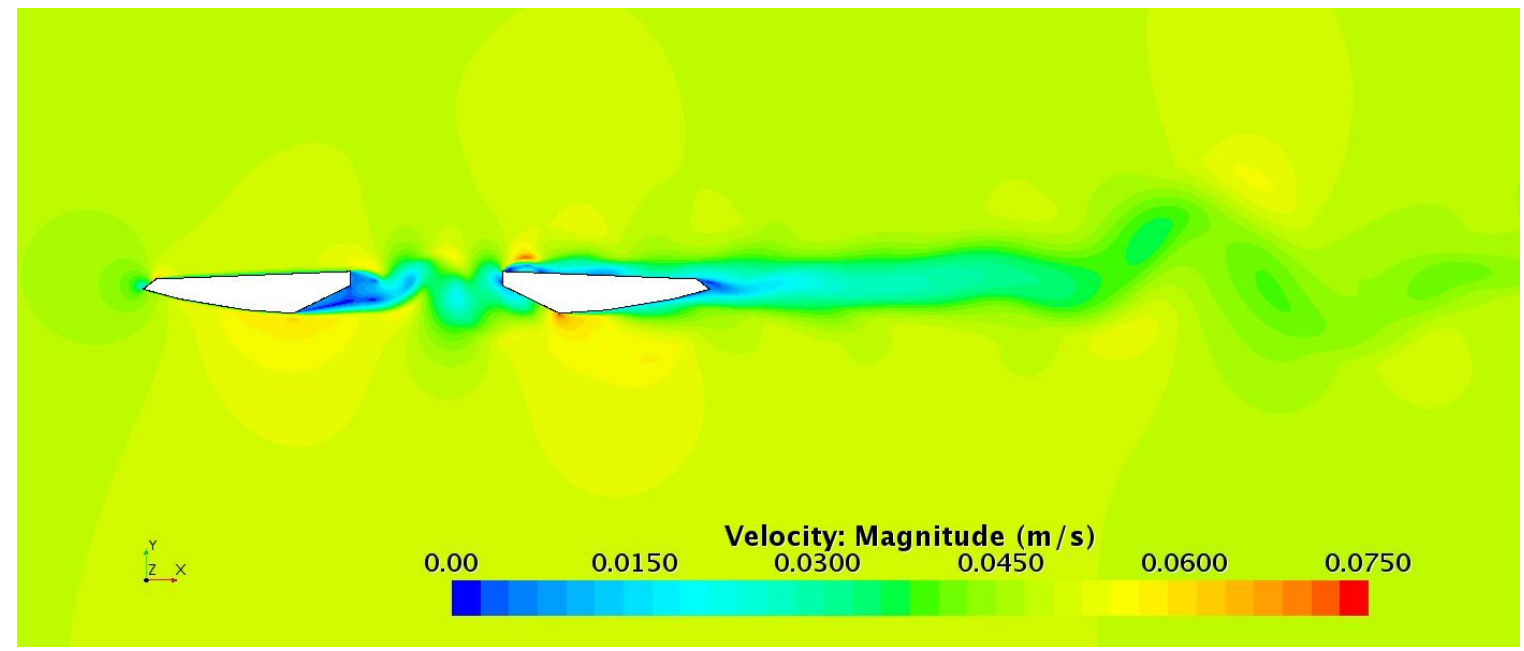

(b)

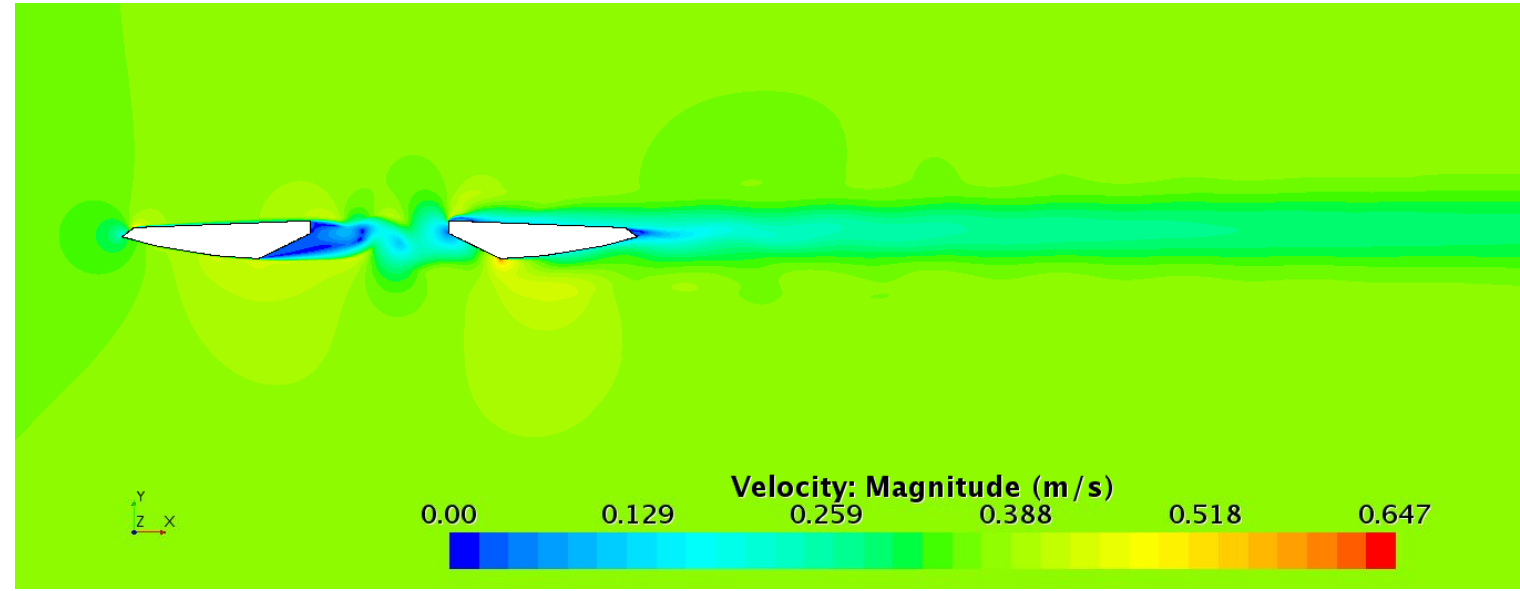

Figure 6-7: Velocity fields on a vertical plane at (a) $R e=1.2 e 4$, (b) 9.1e4

(a)

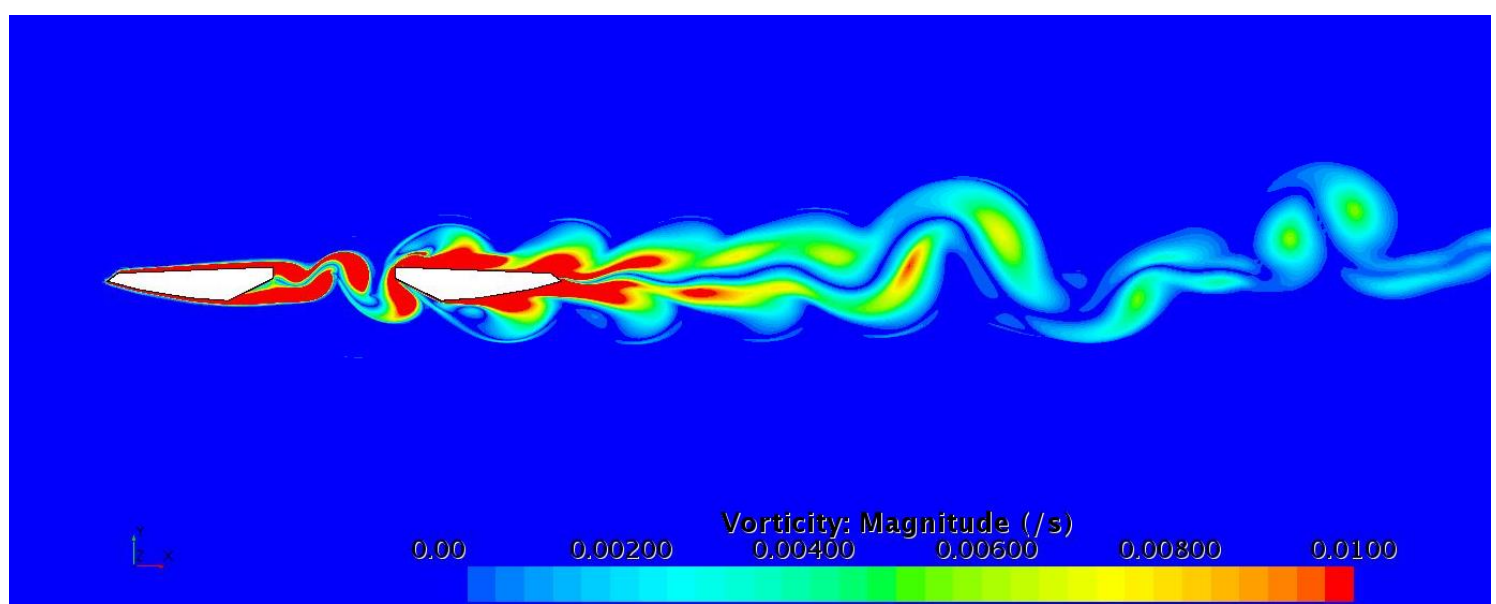


(b)

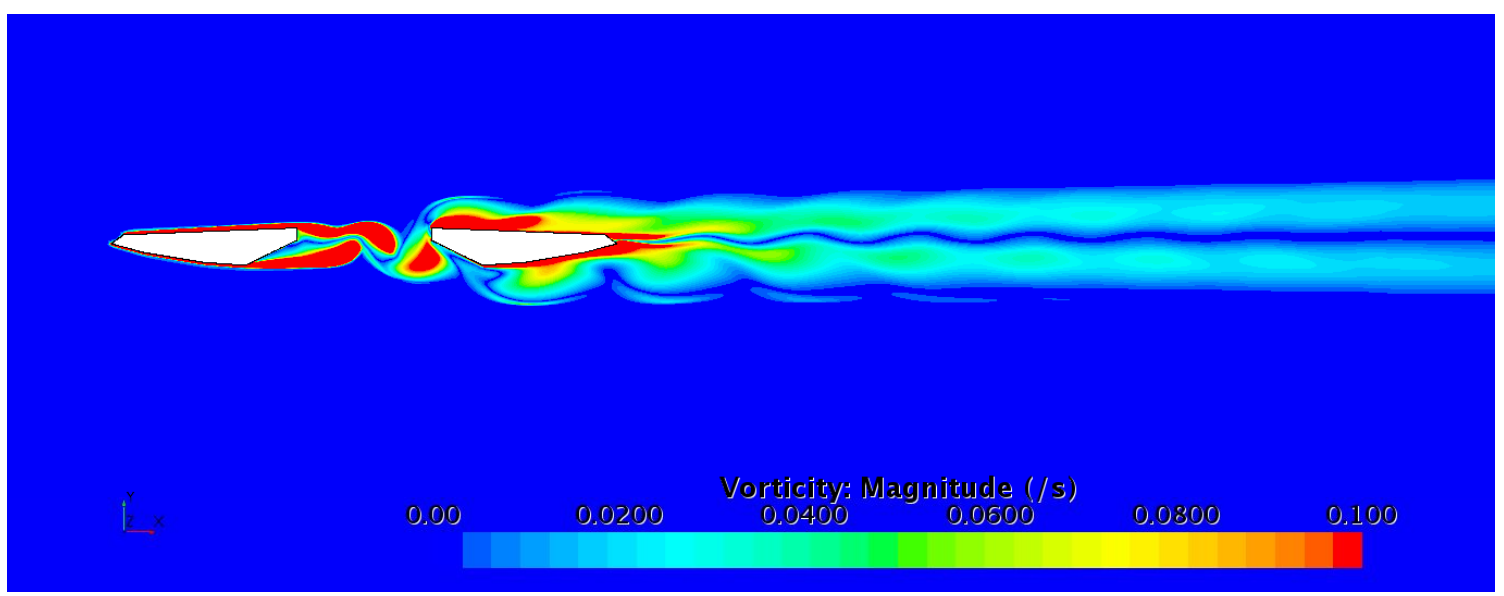

Figure 6-8: Vorticity fields on a vertical plane at (a) $\operatorname{Re}=1.2 \mathrm{e} 4$, (b) $9.1 \mathrm{e} 4$

The static force coefficients are calculated according to the formulas presented at the beginning of the chapter, equations (1), (2), and (3). The Strouhal number is calculated as follows:

$$
S t=\frac{f_{s} H}{U},
$$

where $f_{s}$ is the vortex shedding frequency. The vortex shedding frequency was derived from the period of the oscillating lift force and is equal $0.00312 \mathrm{~Hz}$ at $\mathrm{Re}=12,000$, and $0.0223 \mathrm{~Hz}$ at $\mathrm{Re}=91,000$.

Table 6-1: Force coefficients and Strouhal number obtained from CFD simulations

\begin{tabular}{|l|c|c|c|c|}
\hline & \multicolumn{2}{|c|}{ Re $=\mathbf{1 . 2 e} 4$} & \multicolumn{2}{c|}{ Re $=$ 9.1e4 } \\
\hline & CFD & Ref. $[18],[25]$ & CFD & Ref. [18], [25] \\
\hline $\mathbf{C}_{\mathbf{D}}$ & 0.051 & 0.048 & 0.045 & 0.050 \\
\hline $\mathbf{C}_{\mathbf{L}}$ & -0.125 & -0.048 & -0.115 & -0.110 \\
\hline $\mathbf{C}_{\mathbf{M}}$ & 0.003 & 0.017 & 0.003 & 0.009 \\
\hline $\mathbf{S t}$ & 0.24 & 0.23 & 0.22 & 0.27 \\
\hline
\end{tabular}

The combined computational results of force coefficients and Strouhal number obtained from CFD simulations are presented in Table 6-1. The drag coefficient, as well as the lift coefficient, decrease in absolute value for higher Reynolds number, whereas the moment coefficient doesn't experience any difference. Compared to the numbers found in the literature ([18], [25]) for Re $=1.2 \mathrm{e} 4$, the drag, lift and moment coefficients differ by $6 \%, 160 \%$ and $-82 \%$, respectively. For the higher value of Reynolds number, $\mathrm{Re}=9.1 \mathrm{e} 4$, the coefficients differ by $-10 \%, 4.5 \%$, and $67 \%$. The Strouhal numbers for the two considered Reynolds numbers differ by $4 \%$ and $-19 \%$, respectively. 


\section{Conclusions}

The main goal of the present study is to assess the capabilities of $3 \mathrm{D}$ CFD computations in a parametric study of a twin deck bridge. The aerodynamic forces and response of a rigid section model with a generic cross-section was investigated. Gap-to-width ratios, wind speed, and wind direction were considered using three-dimensional CFD simulations. Static and dynamic responses of the decks were characterized, including computation of steady state aerodynamic forces and pitching moment in URANS as well as LES simulations to capture the formation of large eddies.

Mesh density sensitivity tests revealed that there is strong influence on the results depending on the type of the mesh and wall Y+ value. There are also differences between the unsteady URANS solver with $\mathrm{k}-\varepsilon$ turbulence model and LES computations. A comparison of results led to a conclusion that in stationary computations it will be beneficial to use URANS and LES models in combination with a polyhedral mesh that is denser around the decks, so that wall Y+ is close to one. Polyhedral meshes are relatively easy and efficient to build and they contain significantly fewer cells than a hexahedral mesh for the same accuracy. A low wall $\mathrm{Y}+$ number was chosen to resolve the boundary layer accurately. In dynamic simulations only the URANS model was used in order to save on computational time and resources.

The observed pressure and velocity fields for a single deck serve as a reference for twin-deck models. The study shows that forces acting on the upstream deck in a tandem configuration, regardless of the spacing between decks, are similar to the single deck results. The values of lift and moment on the upstream deck are higher than for a single deck and they are almost constant regardless the gap size. The downstream deck experiences less drag because it is shielded to some extent by the upstream deck. This effect diminishes as the gap increases. For instance, when gapto-width ratio equals 0.13 , the upstream deck is subjected to a $0.415 \mathrm{~N}$ drag force, whereas the downstream deck is subjected to $0.148 \mathrm{~N}$ drag force. At gap-to-width ratio of 0.79 , the upstream deck is subjected to a $0.421 \mathrm{~N}$ drag force, whereas the downstream deck is subjected to $0.241 \mathrm{~N}$ drag force. The drag force acting on the downstream deck increased by $63 \%$. The lift force also increases as the gap between the twin decks gets bigger. The lift force acting on the upstream deck equals $0.472 \mathrm{~N}$ and on the downstream deck $0.11 \mathrm{~N}$, when the gap-to-width ratio is 0.13 . When the gap-to-width ratio equals 0.79 , then the lift force acting on the upstream deck equals $0.451 \mathrm{~N}$ and on the downstream deck $0.135 \mathrm{~N}$. Therefore, the lift force acting on the downstream deck increased by $23 \%$. The pitch moment does not vary significantly for the upstream deck, staying almost constant and equal to $0.086 \mathrm{Nm}$. The absolute value of the pitch moment for the downstream deck increases from $-0.016 \mathrm{Nm}$ at $\mathrm{L} / \mathrm{B}=0.13$, to $-0.032 \mathrm{Nm}$ at $\mathrm{L} / \mathrm{B}=0.79$, which gives a $100 \%$ increase.

The force changes due to varying angle of attack were computed and combined in graphs. The character of the curves representing drag forces acting on both decks is similar. The drag force is always positive and it increases with the increase of the absolute angle of attack, reaching a maximum at the largest absolute angle value. The lift force changes sign depending on the angle of attack. This tendency occurs for the one deck model as well as for the twin deck model. For negative angles it assumes negative values, goes through zero for angles in the range from $-5 \mathrm{deg}$ to $\mathrm{o}$ deg, and reaches positive values for positive angles. The highest value of the lift force was

obtained for the downstream deck at the biggest angle. It is almost two times higher for the 
downstream deck than the upstream deck. Values of the pitch moment are small compared to the force values for the selected range of the flow directions. The pitch moment for the upstream deck reaches a maximum for parallel flow and decreases as the absolute value of the angle of attack increases. The values of the pitch moment for downstream deck are negative for most of the considered angles of incidence.

The aerodynamic response of the decks was investigated with the use of the Dynamic Fluid Body Interaction (DFBI) solver. The URANS solver with the k- $\varepsilon$ turbulence model was used to solve the fluid flow. The model configurations that were taken into account are: both decks are stationary, both decks are suspended on springs; the upstream deck was suspended on springs and the downstream deck was constrained; and the upstream deck was constrained and the downstream deck was suspended on springs. The first two cases are the most common in testing of bridge sections. The latter, are usually not considered. The configuration with stationary decks was used as a reference case in the analysis.

The configuration with both decks on springs was analyzed in more detail. Vertical motion and rotation of the decks were monitored and recorded. A Fast Fourier Transform was used to establish the frequencies of the vertical motion and rotation of the decks, which are $3.14 \mathrm{~Hz}$ and 3.24 Hz, respectively, for both decks. The oscillations differed with regards to the amplitude, which was higher for the downstream deck. The drag and lift forces and pitch moment were recorded for both decks in each configuration.

The drag forces acting on the upstream deck did not experience significant changes between the different configurations of decks on springs. The highest difference is between the stationary decks and configuration 3, where the upstream deck is constrained but not the downstream deck, with the relative difference less than $1 \%$. The lift forces for dynamic simulations increase, compared to the static computations. The biggest difference is $4 \%$ for configuration 2 , where the downstream deck is constrained but the upstream deck is not. The most significant relative difference of was noticed in the pitch moments. They decreased by $11 \%$ in configuration 3 , and by $12 \%$ in configurations 1 and 2 . The influence on the forces acting on the downstream deck are much more pronounced. The drag force stays at the same level, with the differences only up to $1 \%$. The lift force experiences a change between $-7 \%$ (configuration 2) to 19\% (configuration 3 ). The highest lift force occurs when the upstream deck is constrained and the downstream is not (configuration 3). The reason appears to be that when the upstream deck is constrained, the downstream deck moves to more exposed locations in the wake of the upstream deck. When both decks are unconstrained they tend to move in tandem, with the same frequency. The pitch moments increase by up to $25 \%$ in the configuration 2, compared to the stationary decks.

As the comparison of results for different deck configurations shows, the computational model needs to represent the model tested in the laboratory as closely as possible. For different combinations of restraints on the decks, the response of the upstream and downstream decks is different, therefore when comparing with laboratory measurements where dynamic response is enabled, the same type of dynamic response needs to be included in the model. It is worth noticing that there was no significant difference in computational time between the simulations with one or two DFBI bodies, so there is no additional cost other than setting up the model in including the dynamic response of both decks. 
In conclusion, an extensive study was performed on the influence of air flow around a twin deck bridge section model. The CFD software used provides an array of features that are useful in aerodynamic simulations, assessment of effects of parameter variation and, visualization of results to gain insight into the flow and pressure around twin deck bridge. Modeling of the dynamic response of the decks is also possible using the dynamic fluid body interaction capabilities of the software to analyze the system with the decks mounted on elastic springs, which is very similar to a method of wind tunnel testing of dynamic response.

\section{Acknowledgements}

The funding for this project came from the Wind Research Program at the Turner-Fairbank Highway Research Center, through Interagency Agreement Number DTFH61-14-X-300002 between DOT and DOE, and the work was performed under DOE's contract with UChicago Argonne, LLC, contract no. DE-ACo2-06-CH11357.

\section{References}

[1] Wang, Jie, Jinyun Zhao, and Jianxin Liu. (2012). "Experimental study of aerodynamic interference effects on double thin-walled hollow pier in Tandem arrangement." 4th International Conference on Technology of Architecture and Structure, ICTAS 2011, September 22, 2011 - September 24, 2011. Advanced Materials Research 368-373: 15171520, Trans Tech Publications

[2] Trein, Cristiano Augusto, Hiromichi Shirato, and Masaru Matsumoto. (2015). "On the effects of the gap on the unsteady pressure characteristics of two-box bridge girders." Engineering Structures 82, no. 0: 121-133

[3] Yang, Yongxin, Teng Wu, Yaojun Ge, et al. (2015). "Aerodynamic Stabilization Mechanism of a Twin Box Girder with Various Slot Widths." Journal of Bridge Engineering 20, no. 3

[4] Laima, Shujin, and Hui Li. (2015). "Effects of gap width on flow motions around twin-box girders and vortex-induced vibrations." Journal of Wind Engineering and Industrial Aerodynamics 139: 37-49.

[5] Yang, YongXin, Rui Zhou, and Yao Jun Ge. (2014). "Aerodynamic Flutter Control of Parallel Cable-Stayed Bridges." IABSE Symposium Report, IABSE Madrid Symposium: Engineering for Progress, Nature and People 3190-3197, International Association for Bridge and Structural Engineering.

[6] Chen, Wen-Li, Hui Li, and Hui Hu. (2014). "An experimental study on the unsteady vortices and turbulent flow structures around twin-box-girder bridge deck models with different gap ratios." Journal of Wind Eng. and Industrial Aerodynamics 132: 27-36

[7] Li, Hui, Shujin Laima, and Haiquan Jing. (2014). "Reynolds number effects on aerodynamic characteristics and vortex-induced vibration of a twin-box girder." Journal of Fluids and Structures 50: 358-375 
[8] Belloli, M., F. Fossati, S. Giappino, et al. (2014). "Vortex induced vibrations of a bridge deck: Dynamic response and surface pressure distribution." Journal of Wind Engineering and Industrial Aerodynamics 133, no. 0: 160-168

[9] Li, Hui, Shujin Laima, and Haiquan Jing. (2014). "Reynolds number effects on aerodynamic characteristics and vortex-induced vibration of a twin-box girder." Journal of Fluids and Structures 50: 358-375

[10] Seo, Ju-Won, Ho-Kyung Kim, Jin Park, et al. (2013). "Interference effect on vortexinduced vibration in a parallel twin cable-stayed bridge." Journal of Wind Engineering and Industrial Aerodynamics 116: 7-20.

[11] Corriols, Abraham Sanchez, and Guido Morgenthal. (2014). "Vortex-induced vibrations on cross sections in tandem arrangement." Structural Engineering International: Journal of the International Association for Bridge and Structural Engineering (IABSE) 24, no. 1: 20-26

[12] Laima, Shujin, Hui Li, Wenli Chen, et al. (2013). "Investigation and control of vortexinduced vibration of twin box girders." Journal of Fluids and Structures 39: 205-221.

[13] Wang, Qi, Haili Liao, and Mingshui Li. (2013). "Study on wind resistant performance of multiple spans cable-stayed bridge." 6th European and African Conference on Wind Engineering, EACWE 2013, July 7, 2013 - July 13, 2013: International Association for Wind Engineering (IAWE).

[14] Nieto, F., S. Hernandez, I. Kusano, et al. (2014). "Assessment of the aerodynamic response of bridge decks by means of 2D Reynolds averaged Navier-Stokes simulations." 1oth International Conference on Advances in Fluid Mechanics, AFM 2014, July 1, 2014 - July 3, 2014. WIT Transactions on Engineering Sciences 82: 407-417

[15] Lee, Min-Jae, Si-Chul Kim, Young-Hwa Seo, et al. (2012). "The Yi Sun-sin Bridge: Innovative solutions for suspension bridges." Structural Engineering International: Journal of the International Association for Bridge and Structural Engineering (IABSE) 22, no. 1: 32-35

[16] Liu, Zhiwen, Steve C. S. Cai, and Zhengqing Chen. (2010). "Numerical Simulation of Aerodynamic Interference Effects on Aerostatic Coefficients of Two Bluff Sections in Tandem." The Fifth International Symposium on Computational Wind Engineering (CWE201O), Chapel Hill, North Carolina, USA May 23-27, 2010

[17] Meng, Xiaoliang, Ledong Zhu, and Zhenshan Guo. (2011). "Aerodynamic interference effects and mitigation measures on vortex-induced vibrations of two adjacent cable-stayed bridges." Frontiers of Architecture and Civil Engineering in China 5, no. 4: 510-517

[18] Nieto, F., S. Hernandez, I. Kusano, et al. (2014). "Assessment of the aerodynamic response of bridge decks by means of 2D Reynolds averaged Navier-Stokes simulations." 10th International Conference on Advances in Fluid Mechanics, AFM 2014, July 1, 2014 - July 3, 2014. WIT Transactions on Engineering Sciences 82: 407-417, WITPress

[19] Meng, Xiaoliang, Ledong Zhu, and Zhenshan Guo. (2011). "Aerodynamic interference effects and mitigation measures on vortex-induced vibrations of two adjacent cable-stayed bridges." Frontiers of Architecture and Civil Engineering in China 5, no. 4: 510-517

[20] Kwok, K. C. S., X. R. Qin, C. H. Fok, et al. (2012). "Wind-induced pressures around a sectional twin-deck bridge model: Effects of gap-width on the aerodynamic forces and vortex shedding mechanisms." J. of Wind Eng. and Industrial Aerodynamics 110: 50-61 
[21] Qin, X. R., K. C. S. Kwok, C. H. Fok, et al. (2007). "Wind-induced self-excited vibrations of a twin-deck bridge and the effects of gap-width." Wind and Structures, An International Journal 10, no. 5: 463-479

[22] Fok, Chin Hong. (2006). "Aerodynamic characteristics of long-span twin-deck bridges." Thesis (M.Phil. Civil Engineering), Hong Kong University of Science and Technology

[23] Fok, C.H., K.C.S. Kwok, P.A. Hitchcock, et al. (2006). "Effects of Gap width on a Twindeck Bridge: Part 1 - Vortex Shedding Excitation." Proceedings of 12th AWES Wind Engineering Workshop, Darwin, Australia

[24] Fok, C.H., K.C.S. Kwok, P.A. Hitchcock, et al. (2006). "Effects of Gap-width on a Twin Deck Bridge: Part 2 - Aerodynamic Admittance Functions." Proceedings of 12th AWES Wind Engineering Workshop, Darwin, Australia

[25] Nieto F., Kusano I., Hernandez S., Jurado J.A. (2010) "CFD analysis of the vortexshedding response of a twin-box deck cable-stayed bridge" $5^{\text {th }}$ International Symposium on Computational Wind Engineering, Chapel Hill, North Carolina, USA, May 23-27, 2010

[26] Hui M., Ding Q.S. (2006) "Flutter analysis of Stonecutters bridge" Wind and Structures 9: $125-146$

[27] LS-PrePost online documentation www.lstc.com/lspp/index.shtml

\section{Argonne}

\section{Energy Systems Division}

Argonne National Laboratory

9700 South Cass Avenue

Argonne, IL 60439-4815

www.anl.gov

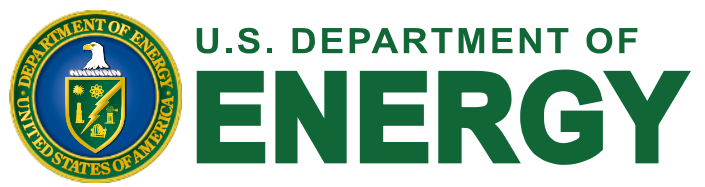

Argonne National Laboratory is a U.S. Department of Energy laboratory managed by UChicago Argonne, LLC 


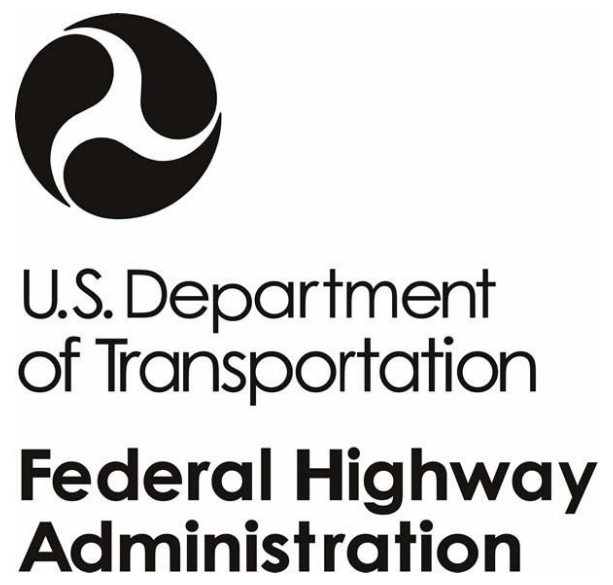

